\title{
Search for nonresonant Higgs boson pair production in final states with two bottom quarks and two photons in proton-proton collisions at $\sqrt{s}=13 \mathrm{TeV}$
}

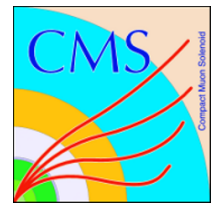

\section{The CMS collaboration}

E-mail: cms-publication-committee-chair@cern.ch

ABSTRACT: A search for nonresonant production of Higgs boson pairs via gluon-gluon and vector boson fusion processes in final states with two bottom quarks and two photons is presented. The search uses data from proton-proton collisions at a center-of-mass energy of $\sqrt{s}=13 \mathrm{TeV}$ recorded with the CMS detector at the LHC, corresponding to an integrated luminosity of $137 \mathrm{fb}^{-1}$. No significant deviation from the background-only hypothesis is observed. An upper limit at $95 \%$ confidence level is set on the product of the Higgs boson pair production cross section and branching fraction into $\gamma \gamma \mathrm{b} \bar{b}$. The observed (expected) upper limit is determined to be $0.67(0.45) \mathrm{fb}$, which corresponds to $7.7(5.2)$ times the standard model prediction. This search has the highest sensitivity to Higgs boson pair production to date. Assuming all other Higgs boson couplings are equal to their values in the standard model, the observed coupling modifiers of the trilinear Higgs boson selfcoupling $\kappa_{\lambda}$ and the coupling between a pair of Higgs bosons and a pair of vector bosons $c_{2 \mathrm{~V}}$ are constrained within the ranges $-3.3<\kappa_{\lambda}<8.5$ and $-1.3<c_{2 \mathrm{~V}}<3.5$ at $95 \%$ confidence level. Constraints on $\kappa_{\lambda}$ are also set by combining this analysis with a search for single Higgs bosons decaying to two photons, produced in association with top quarkantiquark pairs, and by performing a simultaneous fit of $\kappa_{\lambda}$ and the top quark Yukawa coupling modifier $\kappa_{\mathrm{t}}$.

KEYWORDS: Hadron-Hadron scattering (experiments), Higgs physics

ArXiv EPrint: 2011.12373 


\section{Contents}

1 Introduction 1

2 The CMS detector 3

3 Higgs boson pair production $\quad 4$

4 Data sample and simulated events $\quad 6$

$\begin{array}{lll}5 & \text { Event reconstruction and selection } & 7\end{array}$

6 Analysis strategy $\quad 9$

$\begin{array}{lll}7 & \text { The } t \overline{\mathrm{t}} \mathrm{H} \text { background rejection } & 9\end{array}$

8 Nonresonant background rejection $\quad 11$

$\begin{array}{ll}\text { 8.1 Background reduction in the ggF HH signal region } & 11\end{array}$

$\begin{array}{ll}\text { 8.2 Background reduction in the VBF HH signal region } & 12\end{array}$

9 Event categorization $\quad \mathbf{1 4}$

9.1 Combination of the $\mathrm{HH}$ and $\mathrm{t} \overline{\mathrm{t}} \mathrm{H}$ signals to constrain $\kappa_{\lambda}$ and $\kappa_{\mathrm{t}} \quad 15$

$\begin{array}{ll}10 \text { Signal model } & 16\end{array}$

11 Background model $\quad 16$

11.1 Single Higgs background model 16

$\begin{array}{ll}11.2 \text { Nonresonant background model } & 17\end{array}$

$\begin{array}{lr}12 \text { Systematic uncertainties } & 18\end{array}$

13 Results $\quad 20$

14 Summary 26

$\begin{array}{ll}\text { The CMS collaboration } & 36\end{array}$ 


\section{Introduction}

Following the discovery of the Higgs boson $(\mathrm{H})$ by the ATLAS and CMS collaborations [1-3], there has been significant interest in thoroughly understanding the Brout-Englert-Higgs mechanism $[4,5]$. With the last remaining free parameter, the mass of the Higgs boson $\left(m_{\mathrm{H}}\right)$, now measured to be around $125 \mathrm{GeV}$, the Higgs boson self-coupling and the structure of the scalar Higgs field potential are precisely predicted in the standard model (SM). Therefore, measuring the Higgs boson's trilinear self-coupling $\lambda_{\mathrm{HHH}}$ is of particular importance because it provides valuable information for reconstructing the shape of the scalar potential.

At the CERN LHC, the trilinear self-coupling of the Higgs boson is only directly accessible via Higgs boson pair $(\mathrm{HH})$ production. This rare process dominantly occurs via gluon-gluon fusion ( $\mathrm{ggF}$ ). Vector boson fusion ( $\mathrm{VBF}$ ) is the second largest production mode. In the SM, the ggF production cross section in proton-proton ( $\mathrm{pp}$ ) collisions at $\sqrt{s}=13 \mathrm{TeV}$ is $31.1_{-2.0}^{+1.4} \mathrm{fb}$ [6-12], calculated at next-to-next-to-leading order (NNLO) with the resummation at next-to-next-to-leading-logarithm accuracy and including top-quark mass effects at next-to-leading order (NLO). For VBF, the production cross section is calculated to be $1.73 \pm 0.04 \mathrm{fb}[13-15]$ at next-to-NNLO in quantum chromodynamics (QCD). The uncertainties in the values of the cross sections include variations of the factorisation and renormalisation scales, parton distribution function (PDF), and the value of the strong force coupling constant $\left(\alpha_{\mathrm{S}}\right)$. The cross sections are calculated for $m_{\mathrm{H}}=125 \mathrm{GeV}$.

Contributions from physics beyond the SM (BSM) can significantly enhance the $\mathrm{HH}$ production cross section, as well as change the kinematical properties of the produced Higgs boson pair, and consequently those of the decay products. The modification of the properties of nonresonant $\mathrm{HH}$ production via ggF from BSM effects can be parametrized through an effective Lagrangian that extends the SM one with dimension- 6 operators $[16,17]$. This parametrization results in five couplings: $\lambda_{\mathrm{HHH}}$, the coupling between the Higgs boson and the top quark $\left(y_{\mathrm{t}}\right)$, and three additional couplings not present in the SM. Those three couplings represent contact interactions between two Higgs bosons and two gluons $\left(c_{2 \mathrm{~g}}\right)$, between one Higgs boson and two gluons $\left(c_{\mathrm{g}}\right)$, and between two Higgs bosons and two top quarks $\left(c_{2}\right)$. The Feynman diagrams contributing to ggF HH production at leading order (LO) are shown in figure 1. All five of these couplings are investigated in this analysis.

The VBF HH production mode gives access to $\lambda_{\mathrm{HHH}}$, as well as to the coupling between two vector bosons and the Higgs boson (HVV) and the coupling between a pair of Higgs bosons and a pair of vector bosons (HHVV). The Feynman diagrams contributing to this production mode at $\mathrm{LO}$ are shown in figure 2 . While $\lambda_{\mathrm{HHH}}$ is mainly constrained from measurements of $\mathrm{HH}$ production via $\mathrm{ggF}$, and the $\mathrm{HVV}$ coupling modifier $\left(c_{\mathrm{V}}\right)$ is constrained by measurements of vector boson associated production of a single Higgs boson and the decay of the Higgs boson to a pair of bosons [18], the HHVV coupling modifier $\left(c_{2 \mathrm{~V}}\right)$ is only directly measurable via $\mathrm{VBF} \mathrm{HH}$ production. Anomalous values of $c_{2 \mathrm{~V}}$ are investigated to establish the presence of the HHVV-mediated process as a probe of BSM physics.

Previous searches for nonresonant production of a Higgs boson pair via $\mathrm{ggF}$ were performed by both the ATLAS and CMS collaborations using the LHC data collected 


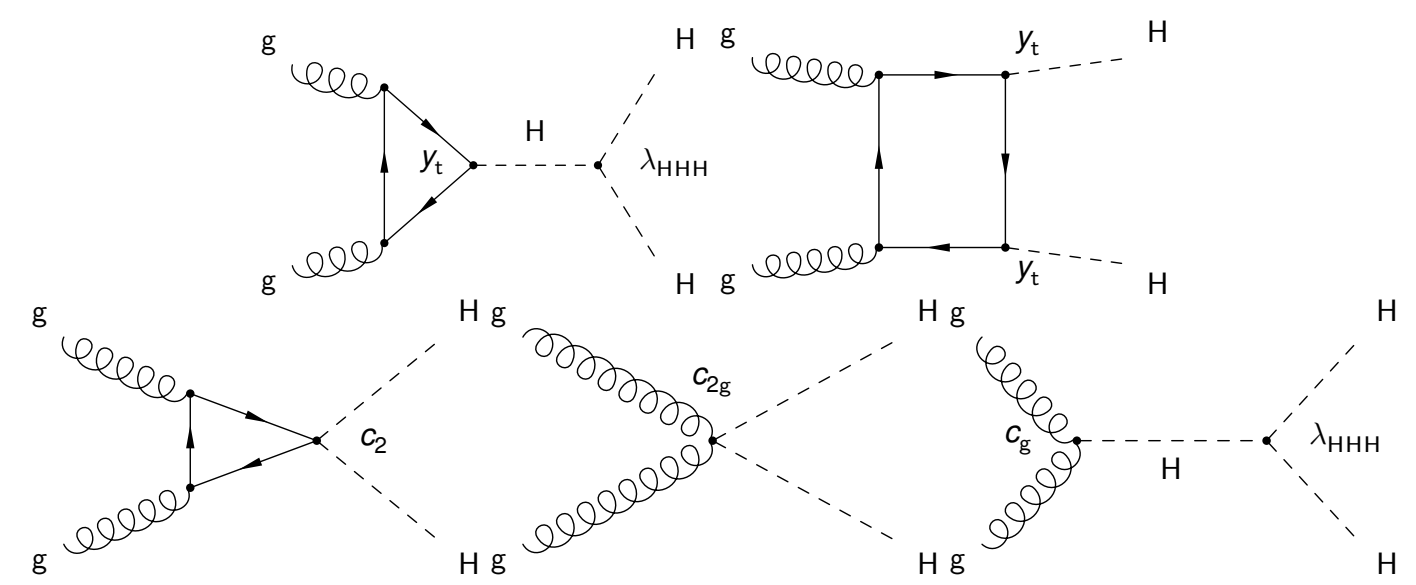

Figure 1. Feynman diagrams of the processes contributing to the production of Higgs boson pairs via ggF at LO. The upper diagrams correspond to SM processes, involving the top Yukawa coupling $y_{\mathrm{t}}$ and the trilinear Higgs boson self-coupling $\lambda_{\mathrm{HHH}}$, respectively. The lower diagrams correspond to BSM processes: the diagram on the left involves the contact interaction of two Higgs bosons with two top quarks $\left(c_{2}\right)$, the middle diagram shows the quartic coupling between the Higgs bosons and two gluons $\left(c_{2 g}\right)$, and the diagram on the right describes the contact interactions between the Higgs boson and gluons $\left(c_{\mathrm{g}}\right)$.
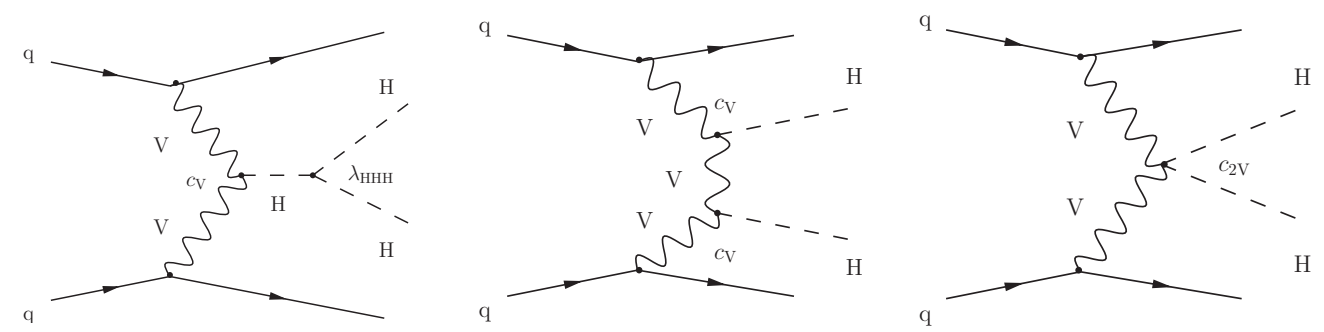

Figure 2. Feynman diagrams that contribute to the production of Higgs boson pairs via VBF at LO. On the left the diagram involving the $\mathrm{HHH}$ vertex $\left(\lambda_{\mathrm{HHH}}\right)$, in the middle the diagram with two $\mathrm{HVV}$ vertices $\left(c_{\mathrm{V}}\right)$, and on the right the diagram with the HHVV vertex $\left(c_{2 \mathrm{~V}}\right)$.

at $\sqrt{s}=8$ and $13 \mathrm{TeV}$ [19-23, 23-29]. Searches in the $\gamma \gamma \mathrm{b} \overline{\mathrm{b}}$ channel performed by the ATLAS [25] and CMS [29] collaborations using up to $36.1 \mathrm{fb}^{-1}$ of pp collision data at $\sqrt{s}=13 \mathrm{TeV}$ set upper limits at $95 \%$ confidence level (CL) on the product of the $\mathrm{HH}$ cross section and the branching fraction into $\gamma \gamma \mathrm{b} \overline{\mathrm{b}}$. The observed upper limits are found to be 24 (30 expected) and 26 (20 expected) times the SM expectation for the ATLAS and CMS searches, respectively. Statistical combinations of search results in various decay channels were also performed by the two experiments $[23,30]$. Recently, the first search for $\mathrm{HH}$ production via VBF was carried out by the ATLAS collaboration in the $b \bar{b} b \bar{b}$ channel [31].

This paper describes a search for nonresonant production of pairs of Higgs bosons decaying to $\gamma \gamma \mathrm{b} \overline{\mathrm{b}}$ using a data sample of $137 \mathrm{fb}^{-1}$ collected by the CMS experiment from 2016 to 2018 . The $\gamma \gamma \mathrm{b} \overline{\mathrm{b}}$ final state has a combined branching fraction of $2.63 \pm 0.06 \times 10^{-3}$ [16] for a Higgs boson mass of $125 \mathrm{GeV}$. This channel is one of the most sensitive to HH produc- 
tion because of the large SM branching fraction of Higgs boson decays to bottom quarks, the good mass resolution of the $\mathrm{H} \rightarrow \gamma \gamma$ channel, and relatively low background rates.

The analysis targets the main $\mathrm{HH}$ production modes: ggF and VBF. Both modes are analyzed following similar strategies. After reducing the nonresonant $\gamma \gamma \mathrm{b} \overline{\mathrm{b}}$ background and the background coming from single Higgs boson production in association with a top quark-antiquark pair ( $\mathrm{t} \overline{\mathrm{t}} \mathrm{H})$, the events are categorized into ggF- and VBF-enriched signal regions using a multivariate technique. The signal is extracted from a fit to the invariant masses of the Higgs boson candidates in the $b \bar{b}$ and $\gamma \gamma$ final states. The analysis described in this paper advances the previous pp $\rightarrow \mathrm{HH} \rightarrow \gamma \gamma \mathrm{b} \overline{\mathrm{b}}$ search [29] by a factor of four, benefiting equally from the larger collected data sets, and the innovative analysis techniques. The enhanced sensitivity of the present analysis was achieved by improving the $b$ jet energy resolution with a dedicated energy regression, introducing new multivariate methods for background rejection, optimizing the event categorization, and adding dedicated VBF categories.

Finally, the search for Higgs boson pair production is combined with an independent analysis that targets $t \bar{t} \mathrm{H}$ production, where the Higgs boson decays to a diphoton pair [32]. The $t \bar{t} \mathrm{H}$ production cross section depends on $y_{\mathrm{t}}$, and also includes a trilinear Higgs boson self-coupling contribution from NLO electroweak corrections [33, 34]. The combination enables $\lambda_{\mathrm{HHH}}$ and $y_{\mathrm{t}}$ to be measured simultaneously and provides constraints applicable to a wide range of theoretical models, where both couplings have anomalous values.

This paper is organized as follows: after a brief description of the CMS detector in section 2, the production of Higgs boson pairs is described in section 3. The data samples and simulation, event reconstruction, and analysis strategy are discussed in sections 4, 5, and 6 , respectively. Sections 7 and 8 are dedicated to the description of the background rejection methods. The event categorization is described in section 9. Sections 10 and 11 describe the modeling of the signal and background, respectively. The systematic uncertainties are discussed in section 12. Finally, the results are presented in section 13. The analysis and its results are then summarized in section 14 .

\section{The CMS detector}

The central feature of the CMS apparatus is a superconducting solenoid of $6 \mathrm{~m}$ internal diameter, providing a magnetic field of $3.8 \mathrm{~T}$. Within the solenoid volume are a silicon pixel and strip tracker, a lead tungstate crystal electromagnetic calorimeter (ECAL), and a brass and scintillator hadron calorimeter (HCAL), each composed of a barrel and two endcap sections. Forward calorimeters extend the pseudorapidity $(\eta)$ coverage provided by the barrel and endcap detectors. Muons are detected in gas-ionization chambers embedded in the steel flux-return yoke outside the solenoid.

A more detailed description of the CMS detector, together with a definition of the coordinate system used and the relevant kinematic variables, can be found in ref. [35].

Events of interest are selected using a two-tiered trigger system [36]. The first level (L1), composed of custom hardware processors, uses information from the calorimeters and muon detectors to select events at a rate of around $100 \mathrm{kHz}$ within a time interval of less 
than $4 \mu \mathrm{s}$. The second level, known as the high-level trigger, consists of a farm of processors running a version of the full event reconstruction software optimised for fast processing, and reduces the event rate to around $1 \mathrm{kHz}$ before data storage [37].

The particle-flow algorithm [38] (PF) aims to reconstruct and identify each individual particle in an event (PF candidate), with an optimised combination of information from the various elements of the CMS detector. The energy of photons is obtained from the ECAL measurement. The energy of electrons is determined from a combination of the track momentum at the main interaction vertex, the corresponding ECAL cluster energy, and the energy sum of all bremsstrahlung photons attached to the track. The momentum of muons is obtained from the curvature of the corresponding track. The energy of charged hadrons is determined from a combination of their momentum measured in the tracker and the matching ECAL and HCAL energy deposits, corrected for zero-suppression effects and for the response function of the calorimeters to hadronic showers. Finally, the energy of neutral hadrons is obtained from the corresponding corrected ECAL and HCAL energies.

For each event, hadronic jets are clustered from these reconstructed particles using the infrared and collinear safe anti- $k_{\mathrm{T}}$ algorithm [39, 40] with a distance parameter of 0.4. Jet momentum is determined as the vectorial sum of all particle momenta in the jet, and is found from simulation to be, on average, within 5 to $10 \%$ of the true momentum over the whole $p_{\mathrm{T}}$ spectrum and detector acceptance. Additional proton-proton interactions within the same or nearby bunch crossings can contribute additional tracks and calorimetric energy depositions, increasing the apparent jet momentum. To mitigate this effect, tracks identified to be originating from pileup vertices are discarded and an offset correction is applied to correct for remaining contributions. Jet energy corrections are derived from simulation studies so that the average measured energy of jets becomes identical to that of particle level jets. In situ measurements of the momentum balance in dijet, photon+jet, $\mathrm{Z}+$ jet, and multijet events are used to determine any residual differences between the jet energy scale in data and in simulation, and appropriate corrections are made [41]. Additional selection criteria are applied to each jet to remove jets potentially dominated by instrumental effects or reconstruction failures. The jet energy resolution amounts typically to $15-20 \%$ at $30 \mathrm{GeV}, 10 \%$ at $100 \mathrm{GeV}$, and $5 \%$ at $1 \mathrm{TeV}$ [41].

The missing transverse momentum vector $\vec{p}_{\mathrm{T}}^{\text {miss }}$ is computed as the negative vector sum of the transverse momenta of all the PF candidates in an event, and its magnitude is denoted as $p_{\mathrm{T}}^{\text {miss }}$ [42]. The $\vec{p}_{\mathrm{T}}^{\text {miss }}$ is modified to account for corrections to the energy scale of the reconstructed jets in the event.

\section{Higgs boson pair production}

Nonresonant $\mathrm{ggF} \mathrm{HH}$ production at the LHC can be described using an effective field theory (EFT) approach [16]. Considering operators up to dimension 6 [17], the tree-level interactions of the Higgs boson are modeled by five parameters. Deviations from the SM values of $\lambda_{\mathrm{HHH}}$ and $y_{\mathrm{t}}$ are parametrized as $\kappa_{\lambda} \equiv \lambda_{\mathrm{HHH}} / \lambda_{\mathrm{HHH}}^{\mathrm{SM}}$ and $\kappa_{\mathrm{t}} \equiv y_{\mathrm{t}} / y_{\mathrm{t}}^{\mathrm{SM}}$, where the $\mathrm{SM}$ values of the couplings are defined as $\lambda_{\mathrm{HHH}}^{\mathrm{SM}} \equiv m_{\mathrm{H}}^{2} /\left(2 v^{2}\right)=0.129, y_{\mathrm{t}}^{\mathrm{SM}}=m_{\mathrm{t}} / v \approx 0.7$. Here, $v=246 \mathrm{GeV}$ is the vacuum expectation value of the Higgs field, and $m_{\mathrm{t}} \approx 173 \mathrm{GeV}$ 


\begin{tabular}{|lrrrrrrrrrrrrr|}
\hline & 1 & 2 & 3 & 4 & 5 & 6 & 7 & 8 & 9 & 10 & 11 & 12 & $\mathrm{SM}$ \\
\hline$\kappa_{\lambda}$ & 7.5 & 1.0 & 1.0 & -3.5 & 1.0 & 2.4 & 5.0 & 15.0 & 1.0 & 10.0 & 2.4 & 15.0 & 1.0 \\
$\kappa_{\mathrm{t}}$ & 1.0 & 1.0 & 1.0 & 1.5 & 1.0 & 1.0 & 1.0 & 1.0 & 1.0 & 1.5 & 1.0 & 1.0 & 1.0 \\
$c_{2}$ & -1.0 & 0.5 & -1.5 & -3.0 & 0.0 & 0.0 & 0.0 & 0.0 & 1.0 & -1.0 & 0.0 & 1.0 & 0.0 \\
$c_{\mathrm{g}}$ & 0.0 & -0.8 & 0.0 & 0.0 & 0.8 & 0.2 & 0.2 & -1.0 & -0.6 & 0.0 & 1.0 & 0.0 & 0.0 \\
$c_{2 \mathrm{~g}}$ & 0.0 & 0.6 & -0.8 & 0.0 & -1.0 & -0.2 & -0.2 & 1.0 & 0.6 & 0.0 & -1.0 & 0.0 & 0.0 \\
\hline
\end{tabular}

Table 1. Coupling parameter values in the SM and in twelve BSM benchmark hypotheses identified using the method described in ref. [44].

is the top quark mass. The anomalous couplings $c_{2 \mathrm{~g}}, c_{2}$, and $c_{\mathrm{g}}$ are not present in the SM. The corresponding part of the Lagrangian can be written as [43]:

$$
\mathcal{L}_{\mathrm{HH}}=\kappa_{\lambda} \lambda_{\mathrm{HHH}}^{\mathrm{SM}} v \mathrm{H}^{3}-\frac{m_{\mathrm{t}}}{v}\left(\kappa_{\mathrm{t}} \mathrm{H}+\frac{c_{2}}{v} \mathrm{H}^{2}\right)\left(\overline{\mathrm{t}}_{\mathrm{L}} \mathrm{t}_{\mathrm{R}}+\text { h.c. }\right)+\frac{1}{4} \frac{\alpha_{\mathrm{S}}}{3 \pi v}\left(c_{\mathrm{g}} \mathrm{H}-\frac{c_{2 \mathrm{~g}}}{2 v} \mathrm{H}^{2}\right) G^{\mu \nu} G_{\mu \nu},
$$

where $t_{L}$ and $t_{R}$ are the top quark fields with left and right chiralities, respectively. The Higgs boson field is denoted as $\mathrm{H}, G^{\mu \nu}$ is the gluon field strength tensor, and h.c. denotes the Hermitian conjugate.

At LO the full cross section of ggF Higgs boson pair production can be expressed by a polynomial with 15 terms corresponding to five individual diagrams, shown in figure 1, and their interference. It has been observed in ref. [44] that twelve benchmark hypotheses, described by various combinations of the five parameters $\left(\kappa_{\lambda}, \kappa_{\mathrm{t}}, c_{2}, c_{\mathrm{g}}, c_{2 \mathrm{~g}}\right)$, are able to represent the distributions of the main kinematic observables of the $\mathrm{HH}$ processes over the full phase space. The parameter values for these benchmark hypotheses are summarized in table 1. The simulated samples generated with the EFT parameters that describe the twelve benchmark hypotheses are combined to cover all possible kinematic configurations of the EFT parameter space. The specific kinematic configurations at any point in the full 5D parameter space are obtained through a corresponding reweighting procedure [44, 45] that parametrizes the changes in the differential ggF HH cross section.

The reweighting procedure described in ref. [44] to obtain the distributions of the kinematic observables is implemented for LO only, and cannot be applied to the higherorder simulation because of the presence of additional partons at the matrix element level. Therefore, the 12 BSM signal benchmark hypotheses summarized in table 1 are investigated using an LO Monte Carlo (MC) simulation, and only anomalous values of $\kappa_{\lambda}$ and $\kappa_{\mathrm{t}}$ are studied with the NLO simulation, as described in section 4 .

In the SM, three different couplings are involved in $\mathrm{HH}$ production via VBF: $\lambda_{\mathrm{HHH}}$, HVV, and HHVV. The Lagrangians corresponding to the left, middle, and right diagrams in figure 2 scale with $c_{\mathrm{V}} \kappa_{\lambda}, c_{\mathrm{V}}^{2}$, and $c_{2 \mathrm{~V}}$, respectively, where $c_{2 \mathrm{~V}}$ and $c_{\mathrm{V}}$ are the HHVV and HVV coupling modifiers, normalized to the SM values. 


\section{Data sample and simulated events}

The analyzed data correspond to a total integrated luminosity of $137 \mathrm{fb}^{-1}$ and were collected over a data-taking period spanning three years: $35.9 \mathrm{fb}^{-1}$ in $2016,41.5 \mathrm{fb}^{-1}$ in 2017 , and $59.4 \mathrm{fb}^{-1}$ in 2018. Events are selected using double-photon triggers with asymmetric thresholds on the photon transverse momenta of $p_{\mathrm{T}}^{\gamma 1}>30 \mathrm{GeV}$ and $p_{\mathrm{T}}^{\gamma 2}>18(22) \mathrm{GeV}$ for the data collected during 2016 (2017 and 2018). In addition, loose calorimetric identification requirements [46], based on the shape of the electromagnetic shower, the isolation of the photon candidate, and the ratio between the hadronic and electromagnetic energy deposit of the shower, are imposed on the photon candidates at the trigger level.

The ggF HH signal samples are simulated at NLO [47-51] including the full top quark mass dependence [52] using POWHEG 2.0. The samples are generated for different values of $\kappa_{\lambda}$. As shown in ref. [49] the dependence of the ggF HH cross section on $\kappa_{\lambda}$ and $\kappa_{\mathrm{t}}$ can be reconstructed from three terms corresponding to the diagrams involving $\kappa_{\lambda}, \kappa_{\mathrm{t}}$ and the interference. Therefore, samples corresponding to any point in the $\left(\kappa_{\lambda}, \kappa_{\mathrm{t}}\right)$ parameter space can be obtained from the linear combination of any three of the generated MC samples with different values of $\kappa_{\lambda}$.

In addition, LO signal samples are generated for the BSM benchmark hypotheses described in section 3 using MADGRAPH5_aMC@NLO v2.2.2 (2016) or v2.4.2 (2017 and 2018) [53-55]. The simulated LO signal samples, corresponding to the 12 BSM benchmark hypotheses, are added together to increase the number of events, and then reweighted to any coupling configuration $\left(\kappa_{\lambda}, \kappa_{\mathrm{t}}, c_{2}, c_{\mathrm{g}}, c_{2 \mathrm{~g}}\right)$ using generator-level information on the HH system.

The VBF HH signal samples are generated at LO [53] using MADGRAPH5_amC@NLO v2.4.2. The simulated samples are generated for different combinations of the coupling modifier values $\left(\kappa_{\lambda}, c_{\mathrm{V}}, c_{2 \mathrm{~V}}\right)$. Similarly to what is done for the $\mathrm{ggF} \mathrm{HH}$ samples generated at NLO, samples corresponding to any point in the $\left(\kappa_{\lambda}, c_{\mathrm{V}}, c_{2 \mathrm{~V}}\right)$ parameter space can be obtained from the linear combination of any six of the generated samples.

We apply a global k-factor to the generated ggF HH and VBF HH signal samples to scale the cross section to NNLO and next-to-NNLO accuracy respectively. The k-factor is obtained for the cross section prediction in the SM and applied to all considered scenarios. The k-factor for the ggF HH cross section depends on the invariant mass of the two Higgs bosons, however, within the region of sensitivity of this analysis, this effect is covered by the total scale uncertainty.

The dominant backgrounds in this search are irreducible prompt diphoton production $(\gamma \gamma+$ jets $)$ and the reducible background from $\gamma+$ jets events, where the jets are misidentified as isolated photons and $\mathrm{b}$ jets. Although these backgrounds are estimated using data-driven methods, simulated samples are used for the training of multivariate discriminants and the optimization of the analysis categories. The $\gamma \gamma+$ jets background is modeled with SHERPA v.2.2.1 [56] at LO and includes up to three additional partons at the matrix element level. In addition, a b-enriched diphoton background is generated with SHERPA at LO requiring up to two b jets to increase the number of simulated events in the analysis region of interest. The $\gamma+$ jets background is modeled with PYTHIA 8.212 [57] at LO. 
Single Higgs boson production, where the Higgs boson decays to a pair of photons, is considered as a resonant background. These production processes are simulated at NLO in QCD precision using POWHeg 2.0 [47, 58-60] for ggF H $(\mathrm{ggH})$ and VBF H, and MAdGRAPH5_amC@NLO v2.2.2 (2016) / v2.4.2 (2017 and 2018) for t $\overline{\mathrm{t}} \mathrm{H}$, vector boson associated production $(\mathrm{VH})$, and production associated with a single top quark. The cross sections and decay branching fractions are taken from ref. [16]. The contribution from the other single $\mathrm{H}$ decay modes is negligible.

All simulated samples are interfaced with PYTHIA for parton showering and fragmentation with the standard $p_{\mathrm{T}}$-ordered parton shower (PS) scheme. The underlying event is modeled with PYTHIA, using the CUETP8M1 tune for 2016 and the CP5 tune for 20172018 [61, 62]. PDFs are taken from the NNPDF3.0 [63] NLO (2016) or NNPDF3.1 [64] NNLO (2017 and 2018) set for all simulated samples except for the signal simulated at LO, for which the PDF4LHC15_NLO_MC set at NLO [63, 65-68] is used. The response of the CMS detector is modeled using the GEANT4 [69] package. The simulated events include additional pp interactions within the same or nearby bunch crossings (pileup), as observed in the data.

Additionally, the simulated VBF HH signal events are also interfaced with the PYTHIA dipole shower scheme to model initial-state radiation (ISR) and final-state radiation (FSR) [70]. The dipole shower scheme correctly takes into account the structure of the color flow between incoming and outgoing quark lines, and its predictions are found to be in good agreement with the NNLO QCD calculations, as reported in ref. [71]. These simulated samples are used to derive the uncertainties associated with the PYTHIA PS ISR and FSR parameters.

\section{Event reconstruction and selection}

The photon candidates are reconstructed from energy clusters in the ECAL not linked to charged-particle tracks (with the exception of converted photons). The photon energies measured by the ECAL are corrected with a multivariate regression technique based on simulation that accounts for radiation lost in material upstream of the ECAL and imperfect shower containment [46]. The ECAL energy scale in data is corrected using simulated $\mathrm{Z} \rightarrow$ ee events, while the photon energy in simulated events is smeared to reproduce the resolution measured in data.

Photons are identified using a boosted decision tree (BDT)-based multivariate analysis (MVA) technique trained to separate photons from jets (photon ID) [46]. The photon ID is trained using variables that describe the shape of the photon electromagnetic shower and the isolation criteria, defined using sums of the transverse momenta of photons, and of charged hadrons, inside a cone of radius $\Delta R=\sqrt{(\Delta \eta)^{2}+(\Delta \phi)^{2}}=0.3$ around the photon candidate direction, where $\phi$ is the azimuthal angle in radians. The imperfect $\mathrm{MC}$ simulation modeling of the input variables is corrected to match the data using a chained quantile regression method [72] based on studies of $\mathrm{Z} \rightarrow$ ee events. In this method, a set of BDTs is trained to predict the cumulative distribution function for a given input. Its prediction is conditional upon the three kinematic variables $\left(p_{\mathrm{T}},|\eta|, \phi\right)$ and the global 
event energy density [46], which are the input variables to the BDTs. The corrections are then applied to the simulated photons such that the predicted cumulative distribution function of the simulated variables is morphed onto the one observed in data.

Events are required to have at least two identified photon candidates that are within the ECAL and tracker fiducial region $(|\eta|<2.5)$, excluding the ECAL barrel-endcap transition region $(1.44<|\eta|<1.57)$ because the reconstruction of a photon object in this region is not optimal. The photon candidates are required to pass the following criteria: $100<m_{\gamma \gamma}<$ $180 \mathrm{GeV}, p_{\mathrm{T}}^{\gamma 1} / m_{\gamma \gamma}>1 / 3$ and $p_{\mathrm{T}}^{\gamma 2} / m_{\gamma \gamma}>1 / 4$, where $m_{\gamma \gamma}$ is the invariant mass of the photon candidates. When more than two photon candidates are found, the photon pair with the highest transverse momentum $p_{\mathrm{T}}^{\gamma \gamma}$ is chosen to construct the Higgs boson candidate.

The primary pp interaction vertex in the event is identified using a multivariate technique based on a BDT following the same approach described in ref. [73]. The BDT is trained on simulated $\mathrm{ggH}$ events and has observables related to tracks recoiling against the identified diphoton system as inputs. The efficiency of the correct vertex assignment is greater than $99.9 \%$, thanks to the requirement of at least two jets in the $\gamma \gamma \mathrm{b} \overline{\mathrm{b}}$ final state.

Jet candidates are required to have $p_{\mathrm{T}}>25 \mathrm{GeV}$ and $|\eta|<2.4$ (2.5) for 2016 (2017-2018) and to be separated from the identified photons by a distance of $\Delta R_{\gamma \mathrm{j}} \equiv$ $\sqrt{\left(\Delta \eta_{\gamma \mathrm{j}}\right)^{2}+\left(\Delta \phi_{\gamma \mathrm{j}}\right)^{2}}>0.4$. The jet $\eta$ range is extended for the 2017 and 2018 data-taking years because of the new CMS pixel detector installed during the Phase-1 upgrade [74]. In addition, identification criteria are applied to remove spurious jets associated with calorimeter noise [75]. Jets from the hadronization of $\mathrm{b}$ quarks are tagged by a secondary vertex algorithm, DeEPJet, based on the score from a deep neural network (DNN) [76, 77]. We will refer to the output of this DNN as the b tagging score.

In addition to standard CMS jet energy corrections [78], a b jet energy regression [79] is used to improve the energy resolution of $\mathrm{b}$ jets and, therefore, the $m_{\mathrm{jj}}$ resolution. The energy correction and resolution estimator are computed for each of the Higgs boson candidate jets through a regression implemented in a DNN and trained on jet properties. The regression simultaneously provides a b jet energy correction and a resolution estimator.

In events with more than two jets, the Higgs boson candidate is reconstructed from the two jets with the highest $\mathrm{b}$ tagging scores. The dijet invariant mass is required to be $70<m_{\mathrm{jj}}<190 \mathrm{GeV}$.

An additional regression was developed specifically for the $\gamma \gamma \mathrm{b} \overline{\mathrm{b}}$ final states to further improve the dijet invariant mass resolution. This regression exploits the fact that there is no genuine missing transverse momentum from the hard-scattering process in the $\gamma \gamma \mathrm{b} \overline{\mathrm{b}}$ final state, and follows a similar approach as used in ref. [29]. The regression targets the dijet invariant mass at the generator level, and is trained using the kinematic properties of the event and $p_{\mathrm{T}}^{\text {miss }}$. The regression is trained on a simulated sample of b-enriched $\gamma \gamma+$ jets events.

The two regression techniques were validated on data collected by the CMS experiment. The two-step regression technique improves the dijet invariant mass resolution of the SM $\mathrm{HH}$ signal by about $20 \%$, and the $m_{\mathrm{jj}}$ peak position is shifted by $5.5 \mathrm{GeV}$ (5\%) closer to the expected Higgs boson mass. 
To select events corresponding to $\mathrm{HH}$ production via $\mathrm{VBF}$, additional requirements are imposed. The VBF process is characterized by the presence of two additional energetic jets, corresponding to two quarks from each of the colliding protons scattered away from the beam line. These "VBF-tagged" jets are expected to have a large pseudorapidity separation, $\left|\Delta \eta_{\mathrm{jj}}^{\mathrm{VBF}}\right|$, and a large dijet invariant mass, $m_{\mathrm{jj}}^{\mathrm{VBF}}$. VBF-tagged jets are required to have $p_{\mathrm{T}}>40$ (30) $\mathrm{GeV}$ for the leading (subleading) jet, $|\eta|<4.7$, and be separated from the selected photon and b jet candidates by $\Delta R_{\gamma \mathrm{j}}>0.4$ and $\Delta R_{\mathrm{bj}}>0.4$. Jets must also pass an identification criterion designed to reduce the number of selected jets originating from pileup [75]. The dijet pair with the highest dijet invariant mass $m_{\mathrm{jj}}^{\mathrm{VBF}}$ is selected as the two VBF-tagged jets. We will refer to these requirements as "VBF selection criteria".

\section{Analysis strategy}

To improve the sensitivity of the search, MVA techniques are used to distinguish the ggF and VBF HH signal from the dominant nonresonant background. The output of the MVA classifiers is then used to define mutually exclusive analysis categories targeting VBF and $\mathrm{ggF} \mathrm{HH}$ production. The $\mathrm{HH}$ signal is extracted from a fit to the invariant masses of the two Higgs boson candidates in the $\left(m_{\gamma \gamma}, m_{\mathrm{jj}}\right)$ plane simultaneously in all categories.

We study the properties of the HH system, built from the reconstructed diphoton and dijet candidates, to identify observables that can help us distinguish between the signal and background. The invariant mass distributions are shown in figure 3 for diphoton and dijet pairs in data and in signal and background simulation after imposing the selection criteria described in section 5 . The signal has a peaking distribution in $m_{\gamma \gamma}$ and $m_{\mathrm{jj}}$. The data distribution, dominated by the $\gamma \gamma+$ jets and $\gamma+$ jets backgrounds, exhibits a falling spectrum because of the nonresonant nature of these processes. In this analysis, these characteristics are used to extract the signal via a fit to $m_{\gamma \gamma}$ and $m_{\mathrm{jj}}$.

The distribution of $\widetilde{M}_{\mathrm{X}}$, defined as:

$$
\widetilde{M}_{\mathrm{X}}=m_{\gamma \gamma \mathrm{jj}}-\left(m_{\mathrm{jj}}-m_{\mathrm{H}}\right)-\left(m_{\gamma \gamma}-m_{\mathrm{H}}\right),
$$

where $m_{\gamma \gamma \mathrm{jj}}$ is the invariant mass of the two Higgs boson candidates, is particularly sensitive to different values of the couplings described in section 3 . The $\widetilde{M}_{\mathrm{X}}$ distribution is less dependent on the dijet and diphoton energy resolutions than $m_{\gamma \gamma \mathrm{jj}}$ if the dijet and diphoton pairs originate from a Higgs boson decay [80]. In figure 4, the distribution of $\widetilde{M}_{\mathrm{X}}$ is shown for several BSM benchmark hypotheses affecting ggF HH production (described in table 1) and for different values of $c_{2 \mathrm{~V}}$ affecting the VBF HH production mode. The SM HH process exhibits a broad structure in $\widetilde{M}_{\mathrm{X}}$, induced by the interference between different processes contributing to $\mathrm{HH}$ production and shaped by the analysis selection. The signals with $c_{2 \mathrm{~V}}=0$ and $c_{2 \mathrm{~V}}=2$ have a much harder spectrum than the SM VBF HH signal.

\section{The $t \overline{\mathrm{t}} \mathrm{H}$ background rejection}

Single Higgs boson production is an important resonant background in the $\gamma \gamma \mathrm{b} \overline{\mathrm{b}}$ final state, with $t \bar{t} H$ production being dominant in high purity signal regions. To reduce $t \bar{t} H$ background contamination, a dedicated classifier (ttHScore) was developed. The classifier is 

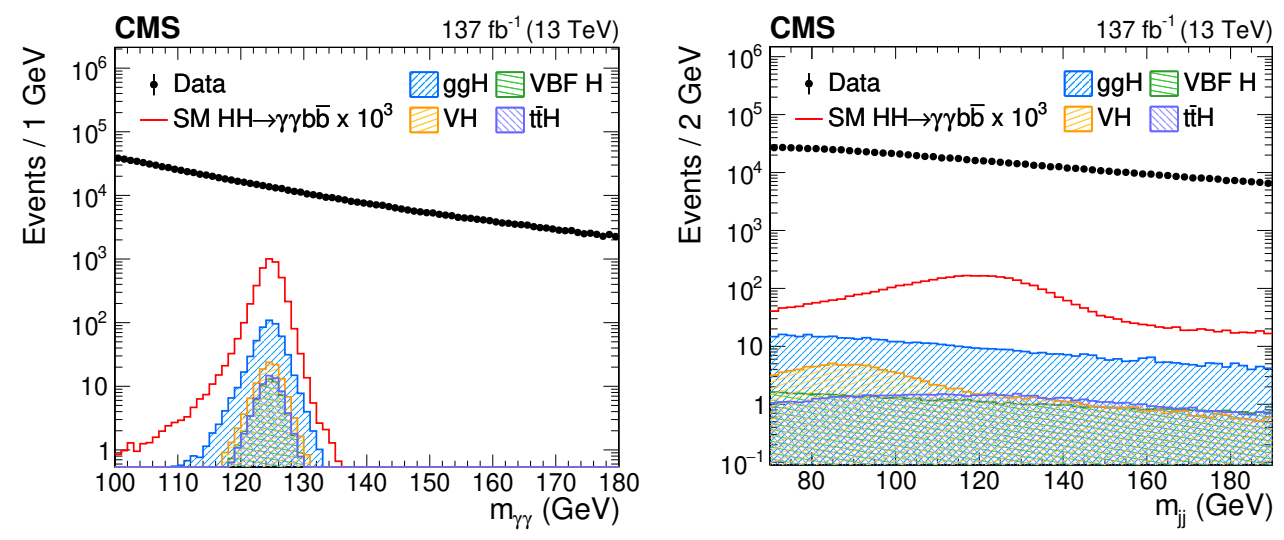

Figure 3. The invariant mass distributions of the reconstructed Higgs boson candidates $m_{\gamma \gamma}$ (left) and $m_{\mathrm{jj}}$ (right) in data and simulated events. Data, dominated by the $\gamma \gamma+$ jets and $\gamma+$ jets backgrounds, are compared to the SM ggF HH signal samples and single $\mathrm{H}$ samples $(\mathrm{t} \overline{\mathrm{t}} \mathrm{H}, \mathrm{ggH}$, VBF H, VH) after imposing the selection criteria described in section 5 . The error bars on the data points indicate statistical uncertainties. The HH signal has been scaled by a factor of $10^{3}$ for display purposes.
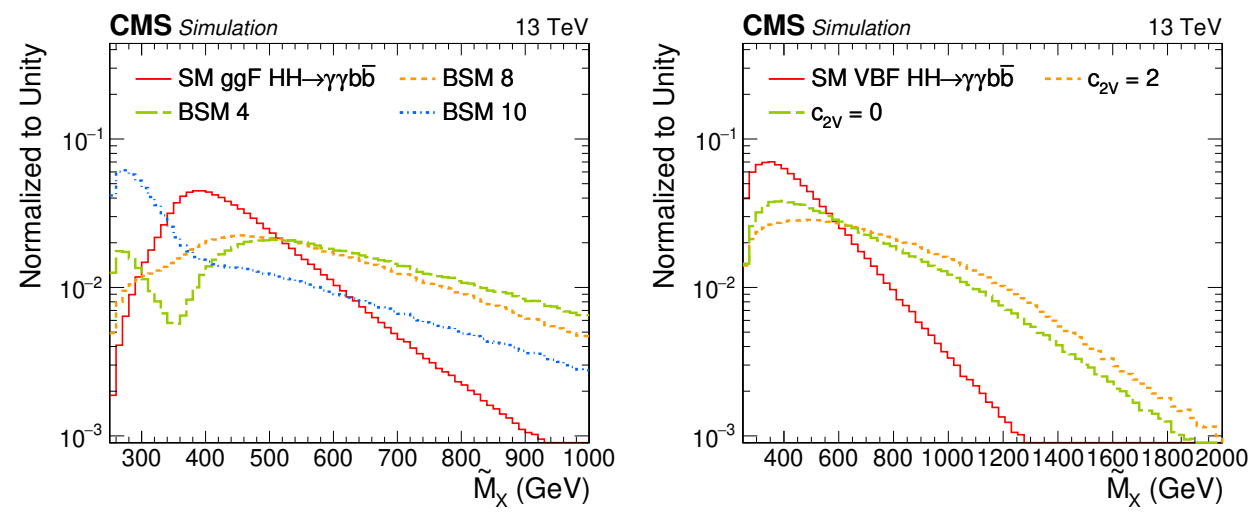

Figure 4. Distributions of $\widetilde{M}_{\mathrm{X}}$. The SM ggF HH signal is compared with several BSM hypotheses listed in table 1 (left), and the SM VBF HH signal is compared with two different anomalous values of $c_{2 \mathrm{~V}}$ (right). All distributions are normalized to unity.

trained on a mixture of SM HH events and events generated for the twelve BSM benchmark hypotheses (described in table 1) as signal, and tet $\mathrm{H}$ events as background. The discriminant uses a combination of low-level information from the individual PF candidates and high-level features describing kinematic properties of the event. The kinematic variables used in the training can be classified in three groups: angular variables, variables to distinguish semileptonic decays of $\mathrm{W}$ bosons produced in the top quark decay, and variables to distinguish hadronic decays of $\mathrm{W}$ bosons. The ttHScore discriminant is implemented with a DNN combining feed-forward and long short-term memory neural networks [81], based on the topology-classifier architecture introduced in ref. [82]. The network is implemented in KeRAS [83] using the TensorFlow [84] backend, and the hyperparameters are chosen through Bayesian optimization. The ttHScore output is shown in figure 5 (left) for data 
and simulated events. The events entering the analysis are required to pass a selection based on this classifier, which is optimized as described in section 9 .

\section{Nonresonant background rejection}

\subsection{Background reduction in the ggF $\mathrm{HH}$ signal region}

An MVA discriminant implemented with a BDT is used to separate the ggF HH signal and the dominant nonresonant $\gamma \gamma+$ jets and $\gamma+$ jets backgrounds. We select several discriminating observables to be used in the training. They can be classified in three groups: kinematic variables, object identification variables, and object resolution variables. The first group exploits the kinematic properties of the HH system, the second helps to separate the signal from the reducible $\gamma+$ jets background, and the third takes into account the resonant nature of the $\gamma \gamma$ and $b \bar{b}$ final states for signal. The following discriminating variables were chosen:

- The $\mathrm{H}$ candidate kinematic variables: $p_{\mathrm{T}}^{\gamma} / m_{\gamma \gamma}, p_{\mathrm{T}}^{\mathrm{j}} / m_{\mathrm{jj}}$ for leading and subleading photons and jets, where $p_{\mathrm{T}}^{\gamma}$ and $p_{\mathrm{T}}^{\mathrm{j}}$ are the transverse momenta of the selected photon and jet candidates.

- The HH transverse balance: $p_{\mathrm{T}}^{\gamma \gamma} / m_{\gamma \gamma \mathrm{jj}}$ and $p_{\mathrm{T}}^{\mathrm{jj}} / m_{\gamma \gamma \mathrm{jj}}$, where $p_{\mathrm{T}}^{\gamma \gamma}$ and $p_{\mathrm{T}}^{\mathrm{jj}}$ are the transverse momenta of the diphoton and dijet candidates.

- Helicity angles: $\left|\cos \theta_{\mathrm{HH}}^{\mathrm{CS}}\right|,\left|\cos \theta_{\mathrm{jj}}\right|,\left|\cos \theta_{\gamma \gamma}\right|$, where $\left|\cos \theta_{\mathrm{HH}}^{\mathrm{CS}}\right|$ is the Collins-Soper angle [85] between the direction of the $\mathrm{H} \rightarrow \gamma \gamma$ candidate and the average beam direction in the HH center-of-mass frame, while $\left|\cos \theta_{\mathrm{jj}}\right|$ and $\left|\cos \theta_{\gamma \gamma}\right|$ are the angles between one of the Higgs boson decay products and the direction defined by the Higgs boson candidate.

- Angular distance: minimum $\Delta R_{\gamma \mathrm{j}}$ between a photon and a jet, $\Delta R_{\gamma \mathrm{j}}^{\min }$, considering all combinations between objects passing the selection criteria, and $\Delta R_{\gamma \mathrm{j}}$ between the other photon-jet pair not used in the $\Delta R_{\gamma j}^{\min }$ calculation.

- $b$ tagging: the $b$ tagging score of each jet in the dijet candidate.

- photon ID: photon identification variables for leading and subleading photons.

- Object resolution: energy resolution for the leading and subleading photons and jets obtained from the photon [46] and b jet [79] energy regressions, the mass resolution estimators for the diphoton and dijet candidates.

The BDT is trained using the XGBOOST [86] software package using a gradient boosting algorithm. The $\gamma \gamma+$ jets and $\gamma+$ jets MC samples are used as background, while an ensemble of SM HH and the 12 BSM HH benchmark hypotheses listed in table 1 is used as signal. Training on an ensemble of BSM and SM HH signals makes the BDT sensitive to a broad spectrum of theoretical scenarios. During the training, signal events are weighted with the product of the inverse mass resolution of the diphoton and dijet systems. These resolutions 

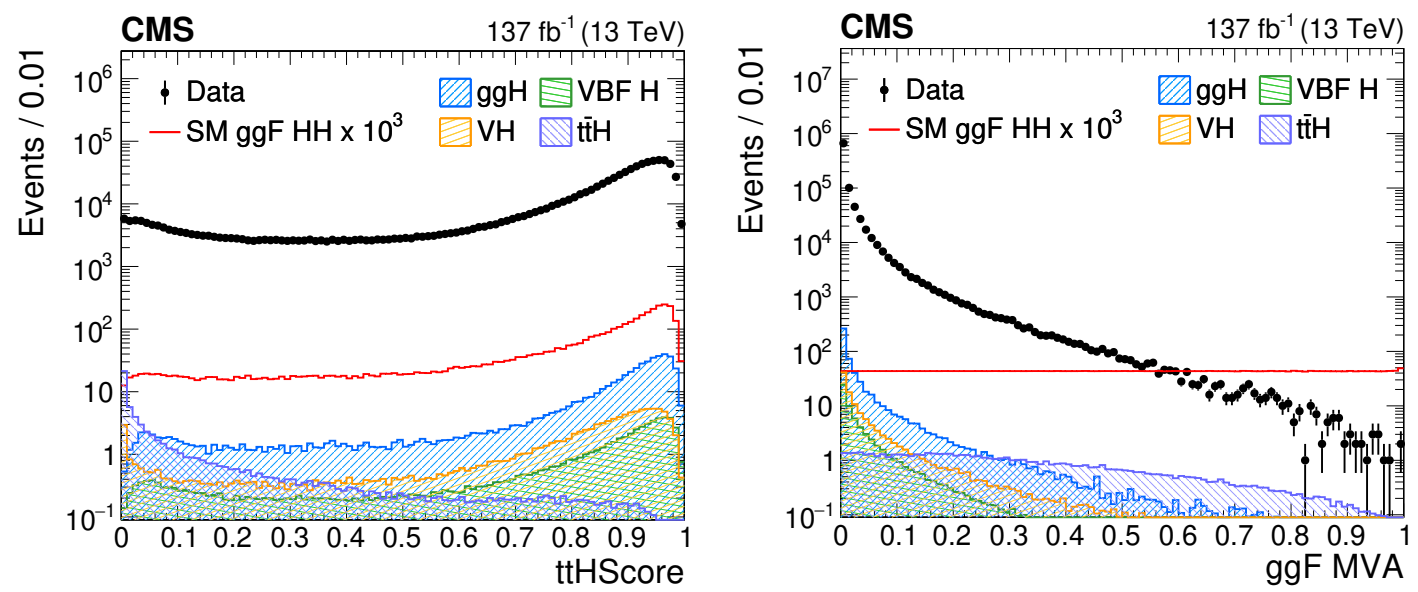

Figure 5. The distribution of the ttHScore (left) and MVA output (right) in data and simulated events. Data, dominated by $\gamma \gamma+$ jets and $\gamma+$ jets background, are compared to the SM ggF $\mathrm{HH}$ signal samples and single $\mathrm{H}$ samples ( $\mathrm{t} \overline{\mathrm{t}} \mathrm{H}, \mathrm{ggH}, \mathrm{VBF} \mathrm{H}, \mathrm{VH})$ after imposing the selection criteria described in section 5 . The error bars on the data points indicate statistical uncertainties. The HH signal has been scaled by a factor of $10^{3}$ for display purposes.

are obtained using the per-object resolution estimators provided by the energy regressions developed for photons and $\mathrm{b}$ jets. In the training, the mass dependence of the classifier is removed by using only dimensionless kinematic variables. The inverse resolution weighting at training time improves the performance by bringing back the information about the resonant nature of the signal. Independent training and testing samples are created by splitting the signal and background samples. The classifier hyperparameters are optimized using a randomized grid search and a 5-fold cross-validation technique [87]. The BDT is trained separately for the 2016, 2017, and 2018 data-taking years. The BDT output distribution is very similar among the three years, leading to the same definitions of optimal signal regions based on the BDT output. Therefore, during the event categorization, a single set of analysis categories is defined using data from 2016-2018. The distributions of the BDT output for signal and background are very well separated. In order to avoid problems of numerical precision when defining optimal signal-enriched regions, the BDT output is transformed such that the signal distribution is uniform. This transformation is applied to all events, both in simulation and data. The distribution of the MVA output for data and simulated events is shown in figure 5 (right).

\subsection{Background reduction in the VBF HH signal region}

Similarly to the ggF HH analysis strategy, an MVA discriminant is employed to separate the VBF HH signal from the background. As for the ggF case, the $\gamma \gamma+$ jets and $\gamma+$ jets processes are the dominant sources of background. For the VBF production mode, the ggF $\mathrm{HH}$ events are considered as background. About a third of the ggF $\mathrm{HH}$ events passing the selection requirements described in section 5 also pass the dedicated VBF selection criteria. The distinctive topology of the VBF HH process is used to separate the VBF HH signal from the various sources of background. In addition to the discriminating features of the 
HH signal described in sections 6 and 8.1, the following set of VBF-discriminating features were identified:

- VBF-tagged jet kinematic variables: $p_{\mathrm{T}}^{\mathrm{VBF}} / m_{\mathrm{jj}}^{\mathrm{VBF}}, \eta^{\mathrm{VBF}}$ for VBF-tagged jets.

- VBF-tagged jet invariant mass: invariant mass $m_{\mathrm{jj}}^{\mathrm{VBF}}$ of the VBF-tagged jets.

- Rapidity gap: product of and difference in the pseudorapidity of the two VBF-tagged jets.

- Quark-gluon likelihood $[88,89]$ of the two VBF-tagged jets. A likelihood discriminator used to distinguish between jets originating from quarks and from gluons.

- Kinematic variables related to the $\mathrm{HH}$ system: $\widetilde{M}_{\mathrm{X}}$ and the transverse momentum of the pair of reconstructed Higgs bosons.

- Angular distance: minimum $\Delta R$ between a photon and a VBF-tagged jet, and between a b jet and a VBF-tagged jet.

- Centrality variables for the reconstructed Higgs boson candidates:

$$
C_{\mathrm{H}}=\exp \left[-\frac{4}{\left(\eta_{1}^{\mathrm{VBF}}-\eta_{2}^{\mathrm{VBF}}\right)^{2}}\left(\eta^{\mathrm{H}}-\frac{\eta_{1}^{\mathrm{VBF}}+\eta_{2}^{\mathrm{VBF}}}{2}\right)^{2}\right],
$$

where $\mathrm{H}$ is the Higgs boson candidate reconstructed either from diphoton or dijet pairs, and $\eta_{1}^{\mathrm{VBF}}$ and $\eta_{2}^{\mathrm{VBF}}$ are the pseudorapidities of the two VBF-tagged jets.

We split events into two regions: $\widetilde{M}_{\mathrm{X}}<500 \mathrm{GeV}$ and $\widetilde{M}_{\mathrm{X}}>500 \mathrm{GeV}$. While the region of $\widetilde{M}_{\mathrm{X}}>500 \mathrm{GeV}$ is sensitive to anomalous values of $c_{2 \mathrm{~V}}$, the $\widetilde{M}_{\mathrm{X}}<500 \mathrm{GeV}$ region retains the sensitivity to SM VBF HH production.

A multi-class BDT, using a gradient boosting algorithm and implemented in the XGBoost [86] framework, is trained to separate the VBF HH signal from the $\gamma \gamma+$ jets, $\gamma+$ jets, and SM ggF HH background. A mix of VBF HH samples with the SM couplings and quartic coupling $c_{2 \mathrm{~V}}=0$ is used as signal. Training on the mix of samples makes the BDT sensitive to both SM and BSM scenarios. Although the kinematic properties of different BSM signals with anomalous values of $c_{2 \mathrm{~V}}$ are similar, the cross section of the signal with $c_{2 \mathrm{~V}}=0$ is significantly enhanced with respect to that predicted by the SM. Therefore, the signal samples used for the training were chosen to maximize sensitivity of the analysis to a range of potential signals. Signal events are weighted with the inverse of the mass resolution of the diphoton and dijet systems during the training, as it is done for the ggF MVA. The BDT is trained separately for each of the three data-taking years in the two $\widetilde{M}_{\mathrm{X}}$ regions. As it is done for the ggF MVA output, data from 2016-2018 are merged to create a single set of analysis categories based on the BDT output. The BDT output is transformed such that the distribution of the mix of the VBF HH signals with $\mathrm{SM}$ couplings and quartic coupling $c_{2 \mathrm{~V}}=0$ is uniform. The transformation is applied to all events in the two $\widetilde{M}_{\mathrm{X}}$ regions. The distribution of the MVA outputs for data and simulated events is shown in figure 6 . 

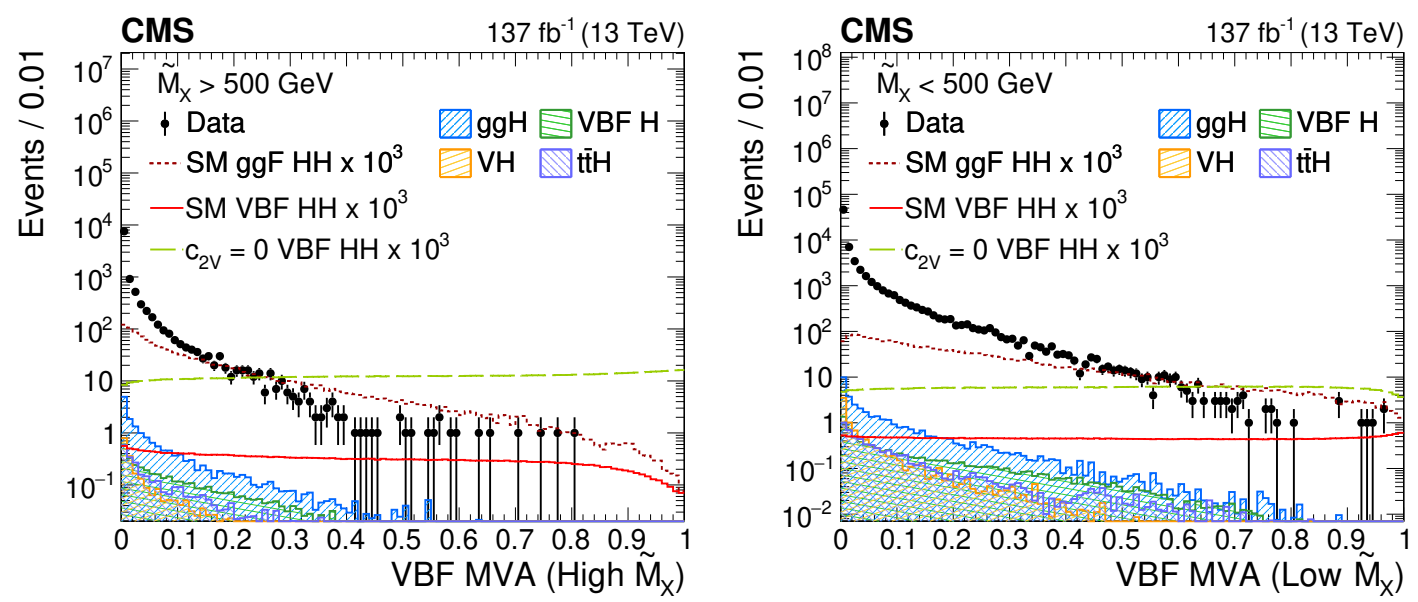

Figure 6. The distribution of the two MVA outputs is shown in data and simulated events in the two VBF $\widetilde{M}_{\mathrm{X}}$ regions: $\widetilde{M}_{\mathrm{X}}>500 \mathrm{GeV}$ (left) and $\widetilde{M}_{\mathrm{X}}<500 \mathrm{GeV}$ (right). Data, dominated by the $\gamma \gamma+$ jets and $\gamma+$ jets backgrounds, are compared to the VBF HH signal samples with SM couplings and $c_{2 \mathrm{~V}}=0, \mathrm{SM} \operatorname{ggF} \mathrm{HH}$ and single $\mathrm{H}$ samples $(\mathrm{t} \overline{\mathrm{t}} \mathrm{H}, \mathrm{ggH}, \mathrm{VBF} \mathrm{H}, \mathrm{VH})$ after imposing the VBF selection criteria described in section 5 . The error bars on the data points indicate statistical uncertainties. The HH signal has been scaled by a factor of $10^{3}$ for display purposes.

\section{Event categorization}

In order to maximize the sensitivity of the search, events are split into different categories according to the output of the MVA classifier and the mass of the Higgs boson pair system $\widetilde{M}_{\mathrm{X}}$. The $\widetilde{M}_{\mathrm{X}}$ distribution changes significantly for different BSM hypotheses, as shown in figure 4. Therefore, a categorization of $\mathrm{HH}$ events in $\widetilde{M}_{\mathrm{X}}$ creates signal regions sensitive to multiple theoretical scenarios. In the search for VBF HH production, the categories in $\widetilde{M}_{\mathrm{X}}$ are defined before the MVA is trained, as described in section 8.2. For the categories that target $\mathrm{ggF} \mathrm{HH}$ production, categories in $\widetilde{M}_{\mathrm{X}}$ are defined after the MVA is trained.

The categorization is optimized by maximizing the expected significance estimated as the sum in quadrature of $\mathrm{S} / \sqrt{\mathrm{B}}$ over all categories in a window centered on $m_{\mathrm{H}}: 115<$ $m_{\gamma \gamma}<135 \mathrm{GeV}$. Here, S and B are the numbers of expected signal and background events, respectively. Simulated events are used for this optimization. The SM HH process is considered as signal, while the background consists of the $\gamma \gamma+$ jets, $\gamma+$ jets, and t $\overline{\mathrm{t}} \mathrm{H}$ processes. The MVA categories are optimized simultaneously with a threshold on the value of ttHScore. Two VBF and three ggF categories are optimized based on the MVA output. For ggF HH in each MVA category a set of $\widetilde{M}_{\mathrm{X}}$ categories is then optimized. The optimization procedure leads to $12 \mathrm{ggF}$ analysis categories: four categories in $\widetilde{M}_{\mathrm{X}}$ in each of the three categories in the MVA score. The optimized selection on ttHScore $>0.26$ corresponds to $80(85) \%$ t $\bar{t} \mathrm{H}$ background rejection at 95 (90)\% signal efficiency for the $12 \mathrm{ggF}(2 \mathrm{VBF})$ categories. The categorization is summarized in table 2. The VBF and ggF categories are mutually exclusive, as we only consider events that do not enter the VBF categories for the ggF categories. Events with VBF MVA scores below 0.52 (0.86) for $\widetilde{M}_{\mathrm{X}}>500\left(\widetilde{M}_{\mathrm{X}}<500\right) \mathrm{GeV}$ are not considered in the VBF signal region. Because 


\begin{tabular}{|lcc|}
\hline Category & MVA & $\widetilde{M}_{\mathrm{X}}(\mathrm{GeV})$ \\
\hline VBF CAT 0 & $0.52-1.00$ & $>500$ \\
VBF CAT 1 & $0.86-1.00$ & $250-500$ \\
$\operatorname{ggF}$ CAT 0 & $0.78-1.00$ & $>600$ \\
ggF CAT 1 & & $510-600$ \\
$\operatorname{ggF}$ CAT 2 & & $385-510$ \\
$\operatorname{ggF}$ CAT 3 & & $250-385$ \\
ggF CAT 4 & $0.62-0.78$ & $>540$ \\
ggF CAT 5 & & $360-540$ \\
ggF CAT 6 & & $330-360$ \\
ggF CAT 7 & & $250-330$ \\
ggF CAT 8 & $0.37-0.62$ & $>585$ \\
ggF CAT 9 & & $375-585$ \\
ggF CAT 10 & & $330-375$ \\
ggF CAT 11 & & $250-330$ \\
\hline
\end{tabular}

Table 2. Summary of the analysis categories. Two VBF- and twelve ggF-enriched categories are defined based on the output of the MVA classifiers and the mass of the Higgs boson pair system $\widetilde{M}_{\mathrm{X}}$. The VBF and ggF categories are mutually exclusive.

of the overwhelming background contamination such events do not improve the expected sensitivity of the analysis. Similarly, events with ggF MVA scores below 0.37 are not considered in the ggF signal region.

\subsection{Combination of the $\mathrm{HH}$ and $\mathrm{t} \overline{\mathrm{t}} \mathrm{H}$ signals to constrain $\kappa_{\lambda}$ and $\kappa_{\mathrm{t}}$}

As discussed in section 3 , the HH production cross section depends on $\kappa_{\lambda}$ and $\kappa_{\mathrm{t}}$. The production cross section of the single $\mathrm{H}$ processes also depends on $\kappa_{\lambda}$, as a result of NLO electroweak corrections [33]. The ggH and $t \bar{t} \mathrm{H}$ production cross sections additionally depend on $\kappa_{\mathrm{t}}$. Therefore, the $\mathrm{HH} \rightarrow \gamma \gamma \mathrm{b} \overline{\mathrm{b}}$ signal can be combined with the single $\mathrm{H}$ production modes to provide an improved constraint on the $\kappa_{\lambda}$ and $\kappa_{\mathrm{t}}$ parameters. In the case of anomalous values of $\kappa_{\lambda}$, the single $\mathrm{H}$ process with the largest modification of the cross section is $t \bar{t} \mathrm{H}$. For this reason, additional orthogonal categories targeting

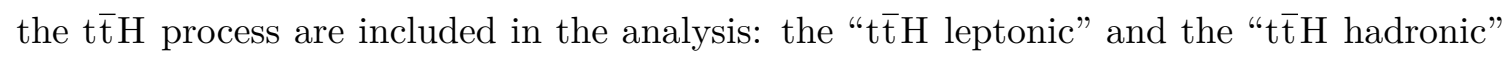
categories, developed and optimized for the measurement of the $t \overline{\mathrm{t}} \mathrm{H}$ production cross section in the diphoton decay channel [32]. The events that do not pass the selections for the $\mathrm{HH}$ categories defined in table 2 are tested for the $t \overline{\mathrm{t}} \mathrm{H}$ categories. This ensures the orthogonality between the events selected by the $\mathrm{HH}$ and $\mathrm{t} \overline{\mathrm{t}} \mathrm{H}$ categories.

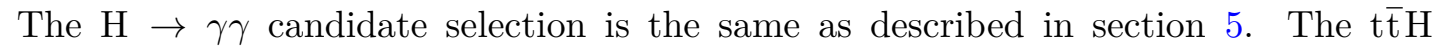
leptonic categories target $\mathrm{t} \overline{\mathrm{t}} \mathrm{H}$ events where at least one $\mathrm{W}$ boson, originating from the top or antitop quark, decays leptonically. At least one isolated electron (muon) with $|\eta|<2.4$ and $p_{\mathrm{T}}>10(5) \mathrm{GeV}$, and at least one jet with $p_{\mathrm{T}}>25 \mathrm{GeV}$ are required. The $\mathrm{t} \overline{\mathrm{t}} \mathrm{H}$ hadronic categories target hadronic decays of $\mathrm{W}$ bosons. In these categories at least three jets are 
required, one of which must be $\mathrm{b}$ tagged, and a lepton veto is imposed. In order to maximize

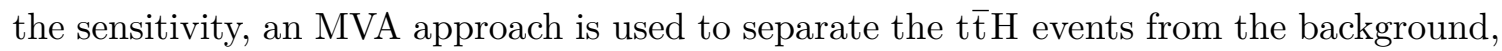
dominated by $\gamma \gamma+$ jets, $\gamma+$ jets, $\mathrm{t} \overline{\mathrm{t}}+$ jets, $\mathrm{t} \overline{\mathrm{t}}+\gamma$, and $\mathrm{t} \overline{\mathrm{t}}+\gamma \gamma$ events. A BDT classifier is trained for each of the two channels using simulated events. The variables used for the training include kinematic properties of the reconstructed objects, object identification variables, and global event properties such as jet and lepton multiplicities. The BDT input variables also include the outputs of other machine learning algorithms trained specifically to target different backgrounds. These include DNN classifiers trained to reduce the $t \overline{\mathrm{t}}+$ $\gamma \gamma$ and $\gamma \gamma+$ jets background, and a top quark tagger based on a BDT [90]. The output scores of the BDTs are used to reject background-like events and to classify the remaining events in four subcategories for each of the two channels. The boundaries of the categories are optimized by maximizing the expected significance of the $t \bar{t} \mathrm{H}$ signal.

\section{Signal model}

In each of the $\mathrm{HH}$ categories, a parametric fit in the $\left(m_{\gamma \gamma}, m_{\mathrm{jj}}\right)$ plane is performed. In the $\mathrm{t} \overline{\mathrm{t}} \mathrm{H}$ categories, the $m_{\gamma \gamma}$ distribution is fitted to extract the signal. When the $\mathrm{HH}$ and $\mathrm{t} \overline{\mathrm{t}} \mathrm{H}$ categories are combined, both the $\mathrm{HH}$ and $\mathrm{t} \overline{\mathrm{t}} \mathrm{H}$ production modes are considered as signals.

The shape templates of the diphoton and dijet invariant mass distributions are constructed from simulation. In each $\mathrm{HH}$ and $\mathrm{t} \overline{\mathrm{t}} \mathrm{H}$ analysis category, the $m_{\gamma \gamma}$ distribution is fitted using a sum of, at most, five Gaussian functions. Figure 7 (left) shows the signal model for $m_{\gamma \gamma}$ in the VBF and ggF CAT0 categories, which are the categories with the best resolution.

For the $\mathrm{HH}$ categories, the $m_{\mathrm{jj}}$ distributions are modeled with a double-sided Crystal Ball (CB) function, a modified version of the standard CB function [91] with two independent exponential tails. Figure 7 (right) shows the signal model for $m_{\mathrm{jj}}$ in the $\mathrm{VBF}$ and $\mathrm{ggF}$ categories with the best resolution.

For the HH signal, the final two-dimensional (2D) signal probability distribution function is a product of the independent $m_{\gamma \gamma}$ and $m_{\mathrm{jj}}$ models. The possible correlations are investigated by comparing the $2 \mathrm{D} m_{\gamma \gamma}-m_{\mathrm{jj}}$ distributions in the simulated signal samples with the 2D probability distributions built as a product of the one-dimensional (1D) ones. With the statistical precision available in this analysis, the correlations have been found to be negligible.

\section{Background model}

\subsection{Single Higgs background model}

The SM single H background shape is constructed from the simulation following the same methodology as used for the signal model described in section 10. For each analysis category and single $\mathrm{H}$ production mode, the $m_{\gamma \gamma}$ distributions are fitted using a sum of, at most, five Gaussian functions. The $m_{\mathrm{jj}}$ modeling in the $\mathrm{HH}$ categories depends on the production mechanism, and a parametrisation is obtained from the simulated distributions: for the $\mathrm{ggH}$ and VBF $\mathrm{H}$ processes, the $m_{\mathrm{jj}}$ distribution is modeled with a Bernstein polynomial; 

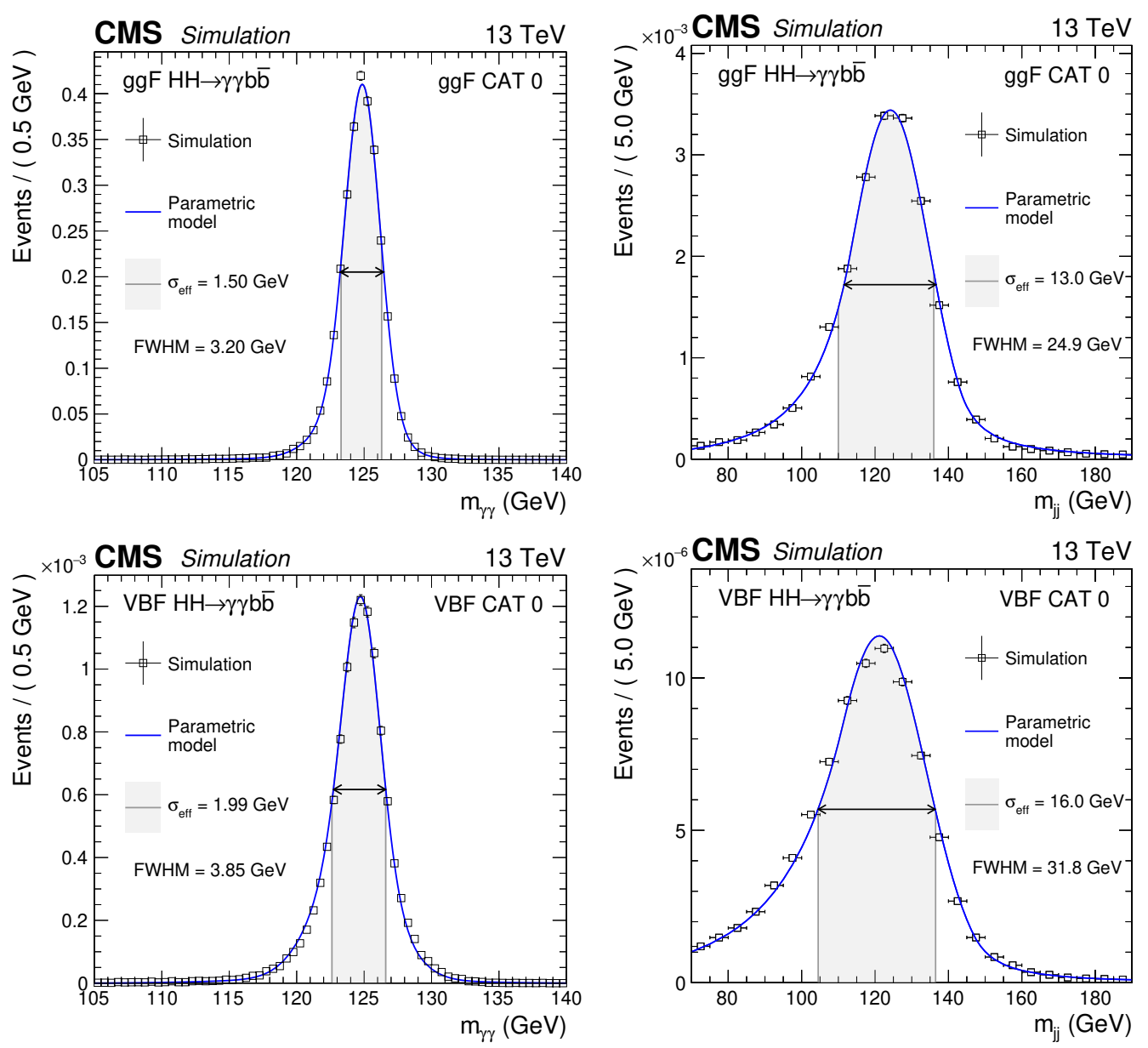

Figure 7. Parametrized signal shape for $m_{\gamma \gamma}$ (left) and $m_{\mathrm{jj}}$ (right) in the best resolution ggF (upper) and VBF (lower) categories. The open squares represent simulated events and the blue lines are the corresponding models. Also shown are the $\sigma_{\text {eff }}$ value (half the width of the narrowest interval containing $68.3 \%$ of the invariant mass distribution) and the corresponding interval as a gray band, and the full width at half the maximum (FWHM) and the corresponding interval as a double arrow.

for $\mathrm{VH}$ production, a CB function is used to model the distribution of the hadronic decays of vector bosons; for $t \bar{t} \mathrm{H}$, where the two $\mathrm{b}$ jets are produced from a top quark decay, a Gaussian function with a mean around $120 \mathrm{GeV}$ is used. Like for the signal modeling, the final 2D SM single $\mathrm{H}$ model is a product of the independent models of the $m_{\gamma \gamma}$ and $m_{\mathrm{jj}}$ distributions.

\subsection{Nonresonant background model}

The model used to describe the nonresonant background is extracted from data using the discrete profiling method [92] as described in ref. [73]. This technique was designed as a way to estimate the systematic uncertainty associated with choosing a particular analytic function to fit the background $m_{\gamma \gamma}$ and $m_{\mathrm{jj}}$ distributions. The method treats the choice of the background function as a discrete nuisance parameter in the likelihood fit to the data. 


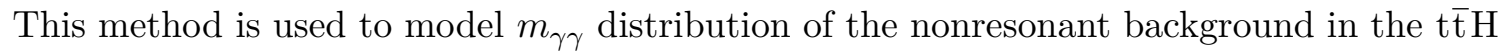
categories. For the $\mathrm{HH}$ categories, the method is generalized to the $2 \mathrm{D}$ model case as a product of two 1D models for $m_{\gamma \gamma}$ and $m_{\mathrm{jj}}$.

A set of MC pseudo-experiments was generated with positive and negative correlations between $m_{\gamma \gamma}$ and $m_{\mathrm{jj}}$ injected and then fitted with the factorized 2D model. A negligible bias has been observed, and the correlations have been found to be within the statistical precision of the analysis.

\section{Systematic uncertainties}

The systematic uncertainties only affect the signal model and the resonant single $\mathrm{H}$ background, since the nonresonant background model is constructed in a data-driven way with the uncertainties associated with the choice of a background fit function taken into account by the discrete profiling method described in section 11.2. The systematic uncertainties can affect the overall normalization, or a variation in category yields, representing event migration between the categories. Theoretical uncertainties have been applied to the $\mathrm{HH}$ and single $\mathrm{H}$ normalizations. The following sources of theoretical uncertainty are considered: the uncertainty in the signal cross section arising from scale variations, uncertainties on $\alpha_{\mathrm{S}}$, PDFs and in the prediction of the branching fraction $\mathcal{B}(\mathrm{HH} \rightarrow \gamma \gamma \mathrm{b} \overline{\mathrm{b}})$. The dominant theoretical uncertainties arise from the prediction of the $\mathrm{SM} \mathrm{HH}$ and $\mathrm{t} \overline{\mathrm{t}} \mathrm{H}$ production cross sections. In addition, a conservative PS uncertainty is assigned to the VBF HH signal, defined as the full symmetrized difference in yields in each category obtained with simulated samples of VBF HH events interfaced with the standard $p_{\mathrm{T}}$-ordered and dipole shower PS schemes.

The dominant experimental uncertainties are:

- Photon identification BDT score: the uncertainty arising from the imperfect MC simulation of the input variables to the photon ID is estimated by rederiving the corrections with equally sized subsets of the $\mathrm{Z} \rightarrow$ ee events used to train the quantile regression BDTs. Its magnitude corresponds to the standard deviation of the eventby-event differences in the photon ID evaluated on the two different sets of corrected input variables. This uncertainty reflects the limited capacity of the BDTs arising from the finite size of the training set. It is seen to cover the residual discrepancies between data and simulation. The uncertainty in the signal yields is estimated by propagating this uncertainty through the full category selection procedure.

- Photon energy scale and resolution: the uncertainties associated with the corrections applied to the photon energy scale in data and the resolution in simulation are evaluated using $\mathrm{Z} \rightarrow$ ee events [93].

- Per-photon energy resolution estimate: the uncertainty in the per-photon resolution is parametrized as a rescaling of the resolution by $\pm 5 \%$ around its nominal value. This is designed to cover all differences between data and simulation in the distribution, which is an output of the energy regression. 
- Jet energy scale and resolution corrections: the energy scale of jets is measured using the $p_{\mathrm{T}}$ balance of jets with $\mathrm{Z}$ bosons and photons in $\mathrm{Z} \rightarrow$ ee, $\mathrm{Z} \rightarrow \mu \mu$, and $\gamma+$ jets events, as well as using the $p_{\mathrm{T}}$ balance between jets in dijet and multijet events $[41,89]$. The uncertainty in the jet energy scale and resolution is a few percent and depends on $p_{\mathrm{T}}$ and $\eta$. The impact of uncertainties on the event yields is evaluated by varying the jet energy corrections within their uncertainties and propagating the effect to the final result. Some sources of the jet energy scale uncertainty are fully (anti-)correlated, while others are considered uncorrelated.

- Jet $b$ tagging: uncertainties in the $\mathrm{b}$ tagging efficiency are evaluated by comparing data and simulated distributions for the $\mathrm{b}$ tagging discriminator [94]. These include the statistical uncertainty in the estimate of the fraction of heavy- and light-flavor jets in data and simulation.

- Trigger efficiency: the efficiency of the trigger selection is measured with $\mathrm{Z} \rightarrow$ ee events using a tag-and-probe technique [95]. An additional uncertainty is introduced to account for a gradual shift in the timing of the inputs of the ECAL L1 trigger in the region $|\eta|>2.0$, which caused a specific trigger inefficiency during 2016 and 2017 data taking. Both photons and, to a greater extent, jets can be affected by this inefficiency, which has a small impact.

- Photon preselection: the uncertainty in the preselection efficiency is computed as the ratio between the efficiency measured in data and in simulation. The preselection efficiency in data is measured with the tag-and-probe technique in $\mathrm{Z} \rightarrow$ ee events [95].

- Integrated luminosity: uncertainties are determined by the CMS luminosity monitoring for the 2016-2018 data-taking years [96-98] and are in the range of 2.3-2.5\%. To account for common sources of uncertainty in the luminosity measurement schemes, some sources are fully (anti-)correlated across the different data-taking years, while others are considered uncorrelated. The total 2016-2018 integrated luminosity has an uncertainty of $1.8 \%$.

- Pileup jet identification: the uncertainty in the pileup jet classification output score is estimated by comparing the score of jets in events with a $\mathrm{Z}$ boson and one balanced jet in data and simulation. The assigned uncertainty depends on $p_{\mathrm{T}}$ and $\eta$, and is designed to cover all differences between data and simulation in the distribution.

Most of the experimental uncertainties are uncorrelated among the three data-taking years. Some sources of uncertainty in the measured luminosity and jet energy corrections are fully (anti-)correlated, while others are considered uncorrelated. This search is statistically limited, and the total impact of systematic uncertainties on the result is about $2 \%$. 


\section{Results}

An unbinned maximum likelihood fit to the $m_{\gamma \gamma}$ and $m_{\mathrm{jj}}$ distributions is performed simultaneously in the $14 \mathrm{HH}$ categories to extract the $\mathrm{HH}$ signal. A likelihood function is defined for each analysis category using analytic models to describe the $m_{\gamma \gamma}$ and $m_{\mathrm{jj}}$ distributions of signal and background events, with nuisance parameters to account for the experimental and theoretical systematic uncertainties described in section 12 . The fit is performed in the mass ranges $100<m_{\gamma \gamma}<180 \mathrm{GeV}$ and $70<m_{\mathrm{jj}}<190 \mathrm{GeV}$ for all categories apart from ggF CAT10 and CAT11. In those two categories, a small but nonnegligible shoulder was observed in the $m_{\mathrm{jj}}$ distribution. Therefore, the $m_{\mathrm{jj}}$ fit range is reduced to $90<m_{\mathrm{jj}}<190 \mathrm{GeV}$ to avoid a possible bias with minimal impact on the analysis sensitivity.

In order to determine $\kappa_{\lambda}$ and $\kappa_{\mathrm{t}}$, the $\mathrm{HH}$ and $\mathrm{t} \overline{\mathrm{t}} \mathrm{H}$ categories are used together in a simultaneous maximum likelihood fit. In the $t \bar{t} H$ categories, a binned maximum likelihood fit is performed to $m_{\gamma \gamma}$ in the mass range $100<m_{\gamma \gamma}<180 \mathrm{GeV}$.

The data and the signal-plus-background model fit to $m_{\gamma \gamma}$ and $m_{\mathrm{jj}}$ are shown in figure 8 for the best resolution $\mathrm{ggF}$ and $\mathrm{VBF}$ categories. The distribution of events weighted by $\mathrm{S} /(\mathrm{S}+\mathrm{B})$ from all $\mathrm{HH}$ categories is shown in figure 9 for $m_{\gamma \gamma}$ and $m_{\mathrm{jj}}$. In this expression, S (B) is the number of signal (background) events extracted from the signal-plusbackground fit.

No significant deviation from the background-only hypothesis is observed. We set upper limits at $95 \% \mathrm{CL}$ on the product of the production cross section of a pair of Higgs bosons and the branching fraction into $\gamma \gamma \mathrm{b} \overline{\mathrm{b}}, \sigma_{\mathrm{HH}} \mathcal{B}(\mathrm{HH} \rightarrow \gamma \gamma \mathrm{b} \overline{\mathrm{b}})$, using the modified frequentist approach for confidence levels $\left(\mathrm{CL}_{\mathrm{s}}\right)$, taking the LHC profile likelihood ratio as a test statistic [99-102] in the asymptotic approximation. The observed (expected) 95\% $\mathrm{CL}$ upper limit on $\sigma_{\mathrm{HH}} \mathcal{B}(\mathrm{HH} \rightarrow \gamma \gamma \mathrm{b} \overline{\mathrm{b}})$ amounts to 0.67 (0.45) fb. The observed (expected) limit corresponds to 7.7 (5.2) times the SM prediction. All results were extracted assuming $m_{\mathrm{H}}=125 \mathrm{GeV}$. We observe a variation smaller than $1 \%$ in both the expected and observed upper limits when using $m_{\mathrm{H}}=125.38 \pm 0.14 \mathrm{GeV}$, corresponding to the most precise measurement of the Higgs boson mass to date [103].

Limits are also derived as a function of $\kappa_{\lambda}$, assuming that the top quark Yukawa coupling is SM-like $\left(\kappa_{\mathrm{t}}=1\right)$. The result is shown in figure 10 . The variation in the excluded cross section as a function of $\kappa_{\lambda}$ is directly related to changes in the kinematical properties of $\mathrm{HH}$ production. At $95 \% \mathrm{CL}, \kappa_{\lambda}$ is constrained to values in the interval $[-3.3,8.5]$, while the expected constraint on $\kappa_{\lambda}$ is in the interval $[-2.5,8.2]$. This is the most sensitive search to date.

Assuming instead that an HH signal exists with the properties predicted by the SM, constraints on $\lambda_{\mathrm{HHH}}$ can be set. The results are obtained both with the HH categories only, and with the $\mathrm{HH}$ categories combined with the $t \overline{\mathrm{t}} \mathrm{H}$ categories in a simultaneous maximum likelihood fit. The HH signal is considered together with the single $\mathrm{H}$ processes $(\mathrm{t} \overline{\mathrm{t}} \mathrm{H}$, ggH, VBF H,VH, and Higgs boson production in association with a single top quark). The cross sections and branching fractions of the $\mathrm{HH}$ and single $\mathrm{H}$ processes are scaled as a function of $\kappa_{\lambda}$, while the top quark Yukawa coupling is assumed to be SM-like, $\kappa_{\mathrm{t}}=1$. 

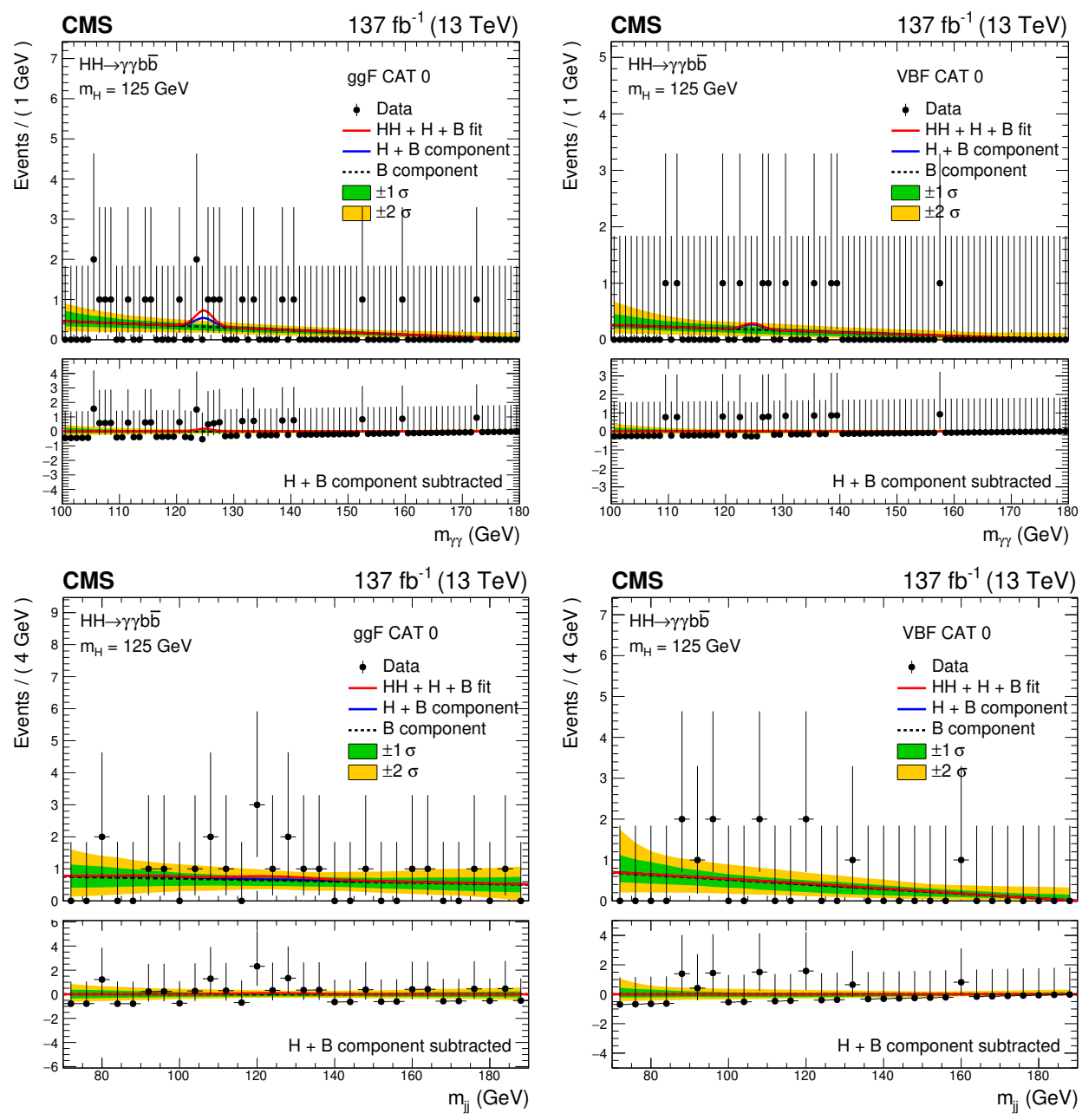

Figure 8. Invariant mass distributions $m_{\gamma \gamma}$ (upper) and $m_{\mathrm{jj}}$ (lower) for the selected events in data (black points) in the best resolution ggF (CAT0) and VBF (CAT0) categories. The solid red line shows the sum of the fitted signal and background $(\mathrm{HH}+\mathrm{H}+\mathrm{B})$, the solid blue line shows the background component from the single Higgs boson and the nonresonant processes $(\mathrm{H}+\mathrm{B})$, and the dashed black line shows the nonresonant background component (B). The normalization of each component (HH, H, B) is extracted from the combined fit to the data in all analysis categories. The one (green) and two (yellow) standard deviation bands include the uncertainties in the background component of the fit. The lower panel in each plot shows the residual signal yield after the background $(\mathrm{H}+\mathrm{B})$ subtraction.

One-dimensional negative log-likelihood scans for $\kappa_{\lambda}$ are shown in figure 11 for an Asimov data set [101] generated with the SM signal-plus-background hypothesis, $\kappa_{\lambda}=1$, and for the observed data. When combining the $\mathrm{HH}$ analysis categories with the $t \overline{\mathrm{t}} \mathrm{H}$ categories, we obtain $\kappa_{\lambda}=0.6_{-1.8}^{+6.3}\left(1.0_{-2.5}^{+5.7}\right.$ expected). Values of $\kappa_{\lambda}$ outside the interval $[-2.7,8.6]$ are excluded at $95 \% \mathrm{CL}$. The expected exclusion at 95\% CL corresponds to the region outside the interval $[-3.3,8.6]$. The shape of the likelihood as function of $\kappa_{\lambda}$ in figure 11 is characterized by 2 minima. This is related to an interplay between the cross section dependence on $\kappa_{\lambda}$ and differences in acceptance between the analysis categories. 

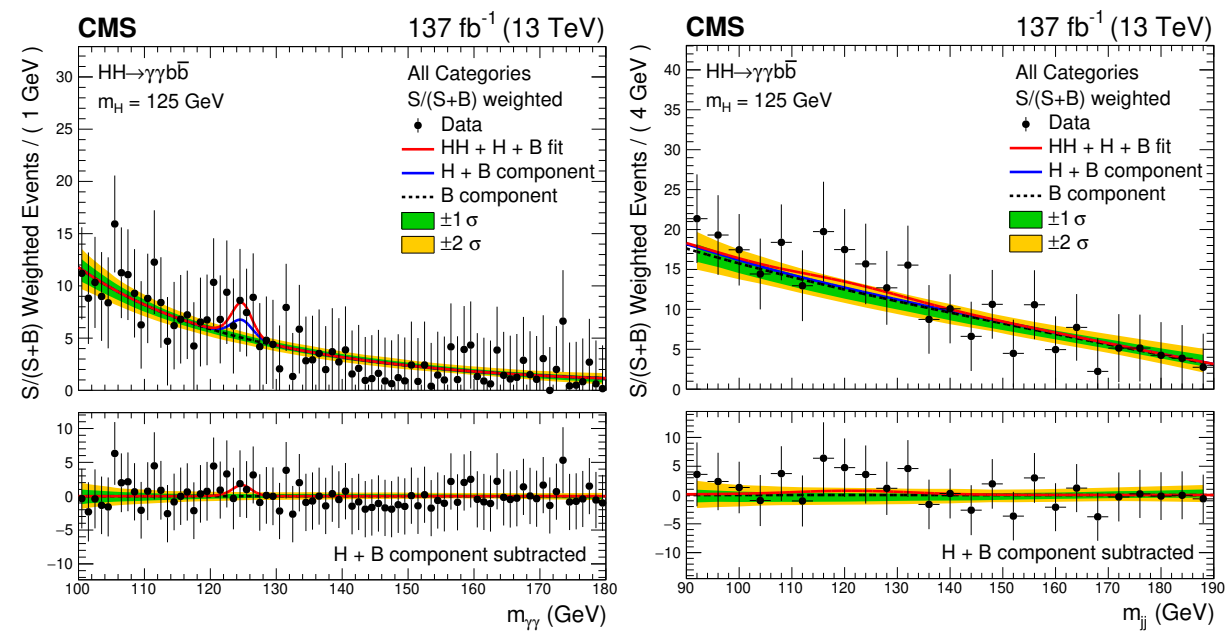

Figure 9. Invariant mass distributions $m_{\gamma \gamma}$ (left) and $m_{\mathrm{jj}}$ (right) for the selected events in data (black points) weighted by $\mathrm{S} /(\mathrm{S}+\mathrm{B})$, where $\mathrm{S}(\mathrm{B})$ is the number of signal (background) events extracted from the signal-plus-background fit. The solid red line shows the sum of the fitted signal and background $(\mathrm{HH}+\mathrm{H}+\mathrm{B})$, the solid blue line shows the background component from the single Higgs boson and the nonresonant processes $(\mathrm{H}+\mathrm{B})$, and the dashed black line shows the nonresonant background component (B). The normalization of each component $(\mathrm{HH}, \mathrm{H}, \mathrm{B})$ is extracted from the combined fit to the data in all analysis categories. The one (green) and two (yellow) standard deviation bands include the uncertainties in the background component of the fit. The lower panel in each plot shows the residual signal yield after the background $(\mathrm{H}+\mathrm{B})$ subtraction.

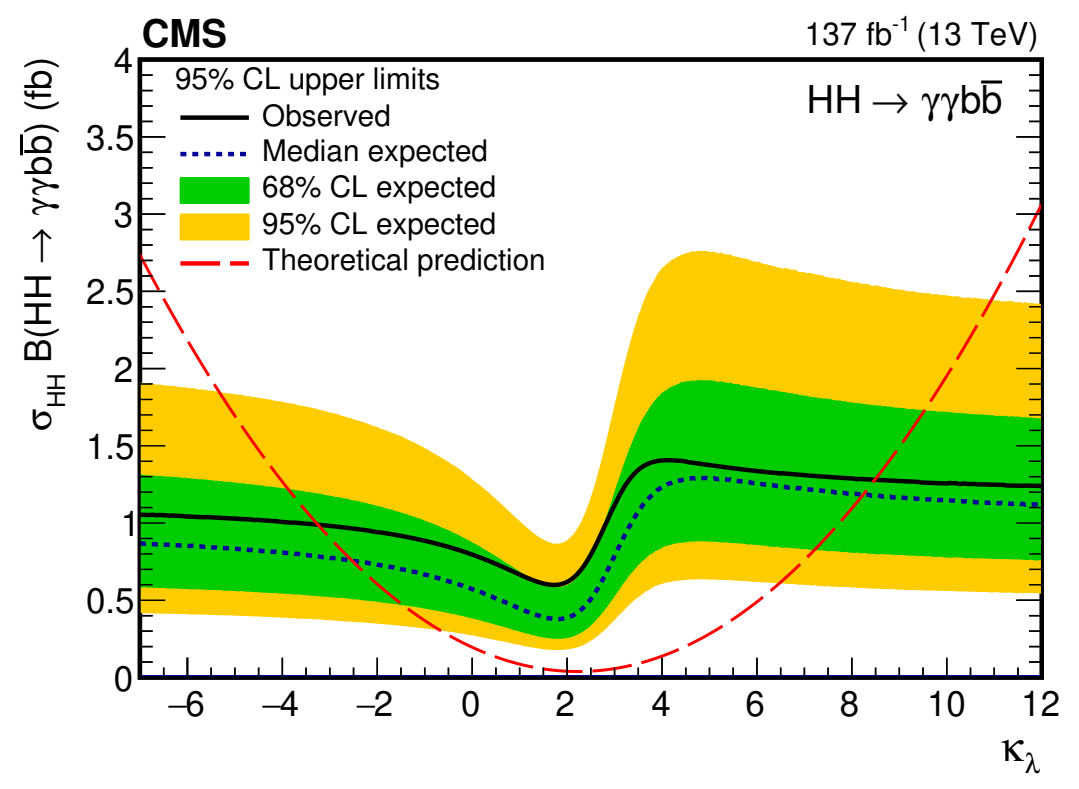

Figure 10. Expected and observed 95\% CL upper limits on the product of the HH production cross section and $\mathcal{B}(\mathrm{HH} \rightarrow \gamma \gamma \mathrm{b} \overline{\mathrm{b}})$ obtained for different values of $\kappa_{\lambda}$ assuming $\kappa_{\mathrm{t}}=1$. The green and yellow bands represent, respectively, the one and two standard deviation extensions beyond the expected limit. The long-dashed red line shows the theoretical prediction. 

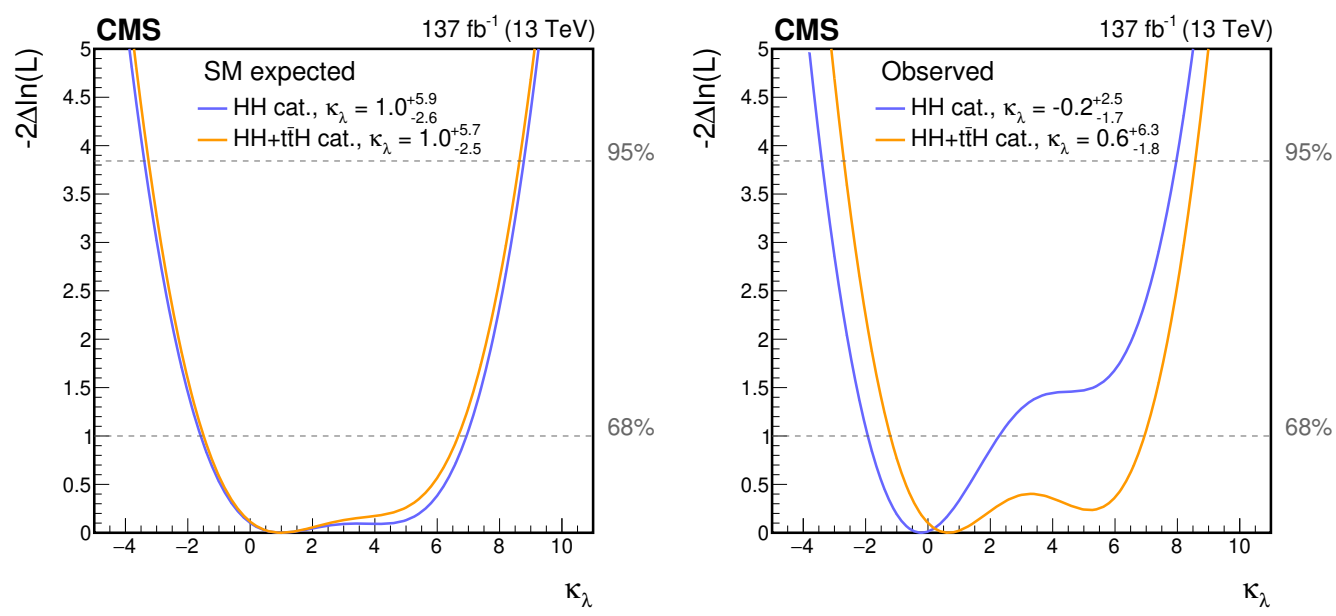

Figure 11. Negative log-likelihood, as a function of $\kappa_{\lambda}$, evaluated with an Asimov data set assuming the SM hypothesis (left) and the observed data (right). The 68 and 95\% CL intervals are shown with the dashed gray lines. The two curves are shown for the $\mathrm{HH}$ (blue) and $\mathrm{HH}+\mathrm{t} \overline{\mathrm{t}} \mathrm{H}$ (orange) analysis categories. All other couplings are set to their SM values.

The HH and single Higgs boson production cross sections depend not only on $\kappa_{\lambda}$, but also on $\kappa_{\mathrm{t}}$. To better constrain the $\kappa_{\lambda}$ and $\kappa_{\mathrm{t}}$ coupling modifiers, a 2D negative loglikelihood scan in the $\left(\kappa_{\lambda}, \kappa_{\mathrm{t}}\right)$ plane is performed, taking into account the modification of the production cross sections and $\mathcal{B}(\mathrm{H} \rightarrow \mathrm{b} \overline{\mathrm{b}}), \mathcal{B}(\mathrm{H} \rightarrow \gamma \gamma)$ for anomalous $\left(\kappa_{\lambda}, \kappa_{\mathrm{t}}\right)$ values. The modification of the single $\mathrm{H}$ production cross section for anomalous $\kappa_{\lambda}$ is modeled at NLO, while the dependence on $\kappa_{\mathrm{t}}$ is parametrized at LO only, neglecting NLO effects [33]. This approximation holds as long as the value of $\left|\kappa_{\mathrm{t}}\right|$ is close to unity, roughly in the range $0.7<\kappa_{\mathrm{t}}<1.3$. The parametric model is not reliable outside of this range. Figure 12 shows the $2 \mathrm{D}$ likelihood scans of $\kappa_{\lambda}$ versus $\kappa_{\mathrm{t}}$ for an Asimov data set assuming the SM hypothesis and for the observed data. The regions of the $2 \mathrm{D}$ scan where the $\kappa_{\mathrm{t}}$ parametrization for anomalous values of $\kappa_{\lambda}$ at LO is not reliable are shown with a gray band.

The inclusion of the $\mathrm{t} \overline{\mathrm{t}} \mathrm{H}$ categories significantly improves the constraint on $\kappa_{\mathrm{t}}$. The $1 \mathrm{D}$ negative log-likelihood scan, as a function of $\kappa_{\mathrm{t}}$ with $\kappa_{\lambda}$ fixed at $\kappa_{\lambda}=1$, is shown in figure 13 for an Asimov data set generated assuming the SM hypothesis, $\kappa_{\mathrm{t}}=1$, as well as for the observed data. The measured value of $\kappa_{\mathrm{t}}$ is $\kappa_{\mathrm{t}}=1.3_{-0.2}^{+0.2}\left(1.0_{-0.2}^{+0.2}\right.$ expected). Values of $\kappa_{\mathrm{t}}$ outside the interval $[0.9,1.9]$ are excluded at $95 \% \mathrm{CL}$. The constraint on $\kappa_{\mathrm{t}}$ is comparable to the one recently set in ref. [104], where anomalous values of $c_{\mathrm{V}}$ were also considered.

Upper limits at 95\% CL are also set on the product of the HH VBF production cross section and branching fraction, $\sigma_{\mathrm{VBF}} \mathrm{HH} \mathcal{B}(\mathrm{HH} \rightarrow \gamma \gamma \mathrm{b} \bar{b})$, with the yield of the ggF $\mathrm{HH}$ signal constrained within uncertainties to the one predicted in the SM. The observed (expected) $95 \% \mathrm{CL}$ upper limit on $\sigma_{\mathrm{VBF}} \mathrm{HH} \mathcal{B}(\mathrm{HH} \rightarrow \gamma \gamma \mathrm{b} \overline{\mathrm{b}})$ amounts to $1.02(0.94) \mathrm{fb}$. The limit corresponds to 225 (208) times the SM prediction. This is the most stringent constraint on $\sigma_{\mathrm{VBF}} \mathrm{HH} \mathcal{B}(\mathrm{HH} \rightarrow \gamma \gamma \mathrm{b} \overline{\mathrm{b}})$ to date. 

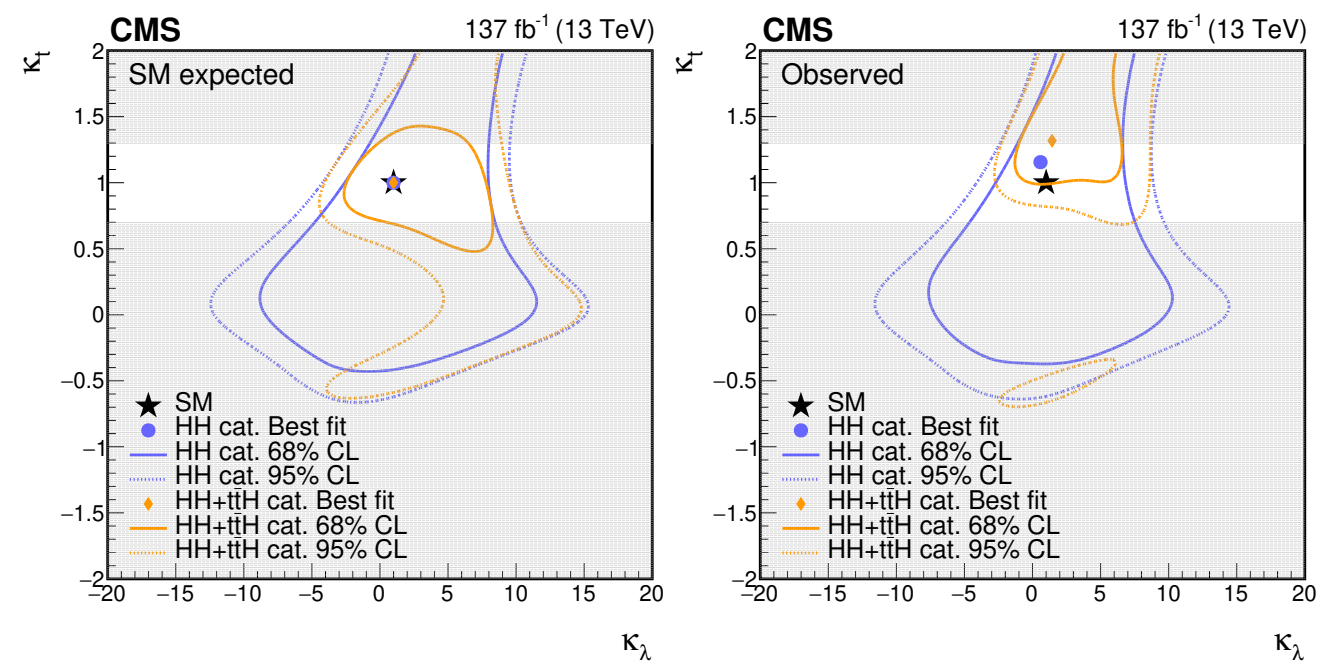

Figure 12. Negative log-likelihood contours at 68 and 95\% CL in the $\left(\kappa_{\lambda}, \kappa_{\mathrm{t}}\right)$ plane evaluated with an Asimov data set assuming the SM hypothesis (left) and the observed data (right). The contours obtained using the $\mathrm{HH}$ analysis categories only are shown in blue, and in orange when combined with the $t \bar{t} H$ categories. The best fit value for the $\mathrm{HH}$ categories only $\left(\kappa_{\lambda}=0.6, \kappa_{\mathrm{t}}=1.2\right)$ is indicated by a blue circle, for the $\mathrm{HH}+\mathrm{t} \overline{\mathrm{t}} \mathrm{H}$ categories $\left(\kappa_{\lambda}=1.4, \kappa_{\mathrm{t}}=1.3\right)$ by an orange diamond, and the SM prediction $\left(\kappa_{\lambda}=1.0, \kappa_{\mathrm{t}}=1.0\right)$ by a black star. The regions of the $2 \mathrm{D}$ scan where the $\kappa_{\mathrm{t}}$ parametrization for anomalous values of $\kappa_{\lambda}$ at LO is not reliable are shown with a gray band.
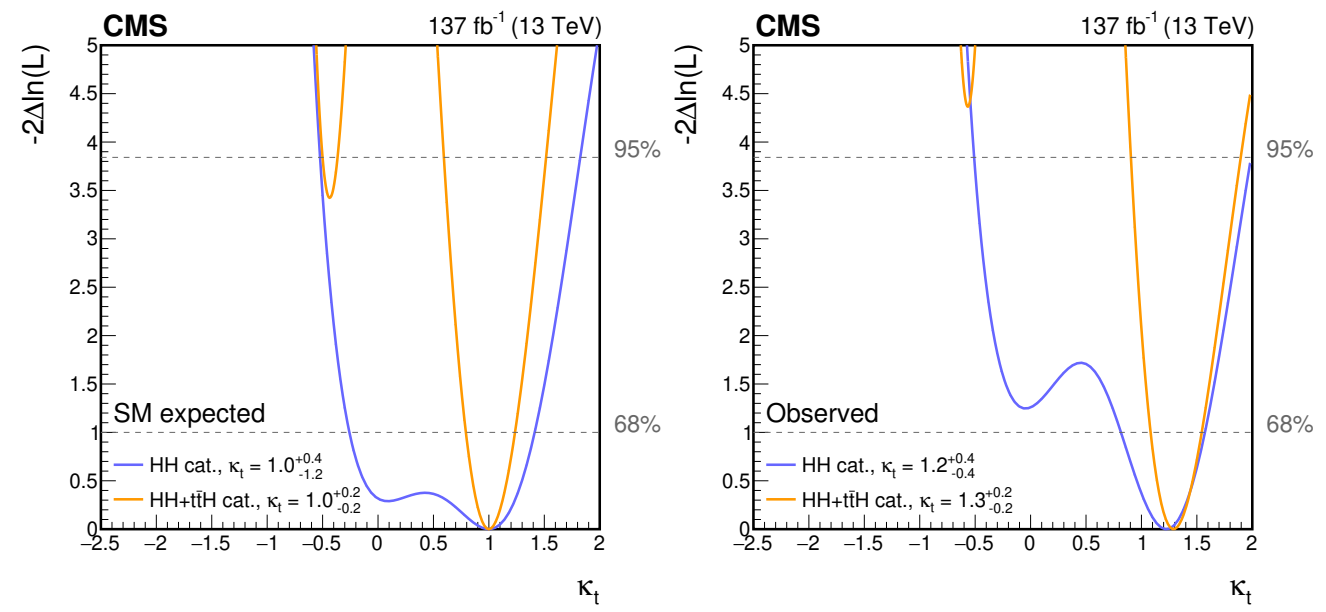

Figure 13. Negative log-likelihood scan, as a function of $\kappa_{\mathrm{t}}$, evaluated with an Asimov data set assuming the SM hypothesis (left) and the observed data (right). The 68 and 95\% CL intervals are shown with the dashed gray lines. The two curves are shown for the $\mathrm{HH}$ (blue) and the $\mathrm{HH}+\mathrm{t} \overline{\mathrm{t}} \mathrm{H}$ (orange) analysis categories. All other couplings are fixed to their SM values. 


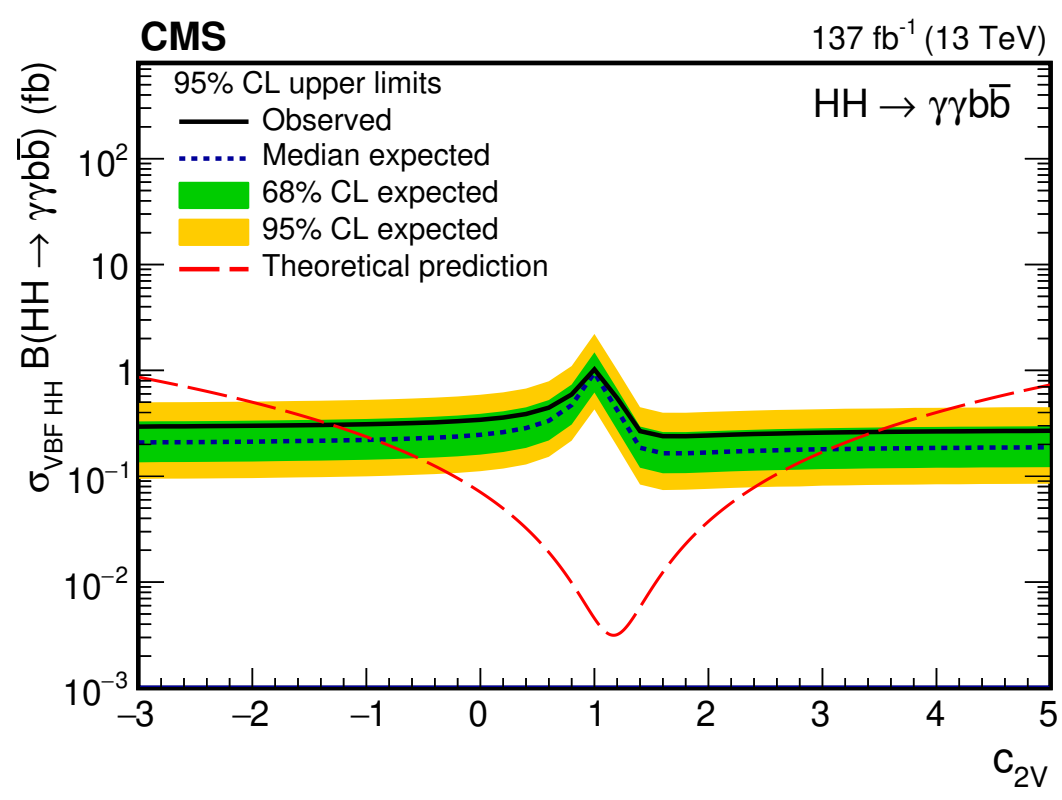

Figure 14. Expected and observed 95\% CL upper limits on the product of the VBF HH production cross section and $\mathcal{B}(\mathrm{HH} \rightarrow \gamma \gamma \mathrm{b} \overline{\mathrm{b}})$ obtained for different values of $c_{2 \mathrm{~V}}$. The green and yellow bands represent, respectively, the one and two standard deviation extensions beyond the expected limit. The long-dashed red line shows the theoretical prediction.

Limits are also set, as a function of $c_{2 \mathrm{~V}}$, as presented in figure 14. The observed excluded region corresponds to $c_{2 \mathrm{~V}}<-1.3$ and $c_{2 \mathrm{~V}}>3.5$, while the expected exclusion is $c_{2 \mathrm{~V}}<-0.9$ and $c_{2 \mathrm{~V}}>3.1$. It can be seen in figure 14 that this analysis is more sensitive to anomalous values of $c_{2 \mathrm{~V}}$ than to the region around the SM prediction. This is related to the fact that, for anomalous values of $c_{2 \mathrm{~V}}$, the total cross section is enhanced and the $\widetilde{M}_{\mathrm{X}}$ spectrum is harder as shown in figure 4 (right). This leads to an increase in the product of signal acceptance and efficiency as well as a more distinct signal topology.

Assuming $\mathrm{HH}$ production occurs via the $\mathrm{VBF}$ and ggF modes, we set constraints on the $\kappa_{\lambda}$ and $c_{2 \mathrm{~V}}$ coupling modifiers simultaneously. A 2D negative log-likelihood scan in the $\left(\kappa_{\lambda}, c_{2 \mathrm{~V}}\right)$ plane is performed using the $14 \mathrm{HH}$ analysis categories. Figure 15 shows $2 \mathrm{D}$ likelihood scans for the observed data and for an Asimov data set assuming all couplings are at their SM values.

We also set upper limits at 95\% CL for the twelve BSM benchmark hypotheses defined in table 1. In this fit, the yield of the VBF HH signal is constrained within uncertainties to the one predicted in the SM. The limits for different BSM hypotheses are shown in figure 16 (upper). In addition, limits are also calculated as a function of the BSM coupling between two Higgs bosons and two top quarks, $c_{2}$, as presented in figure 16 (lower). The observed excluded region corresponds to $c_{2}<-0.6$ and $c_{2}>1.1$, while the expected exclusion is $c_{2}<-0.4$ and $c_{2}>0.9$. 

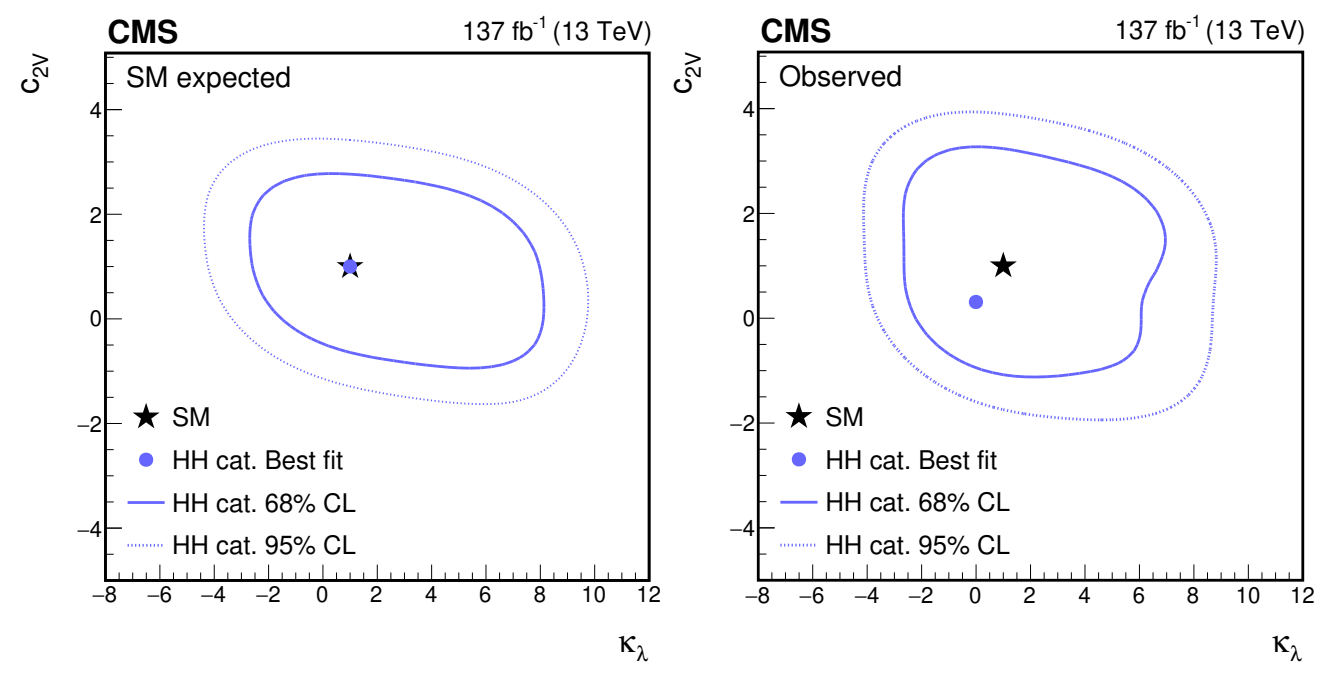

Figure 15. Negative log-likelihood contours at 68 and $95 \%$ CL in the $\left(\kappa_{\lambda}, c_{2 \mathrm{~V}}\right)$ plane evaluated with an Asimov data set assuming the SM hypothesis (left) and with the observed data (right). The contours are obtained using the $\mathrm{HH}$ analysis categories only. The best fit value $\left(\kappa_{\lambda}=0.0, c_{2 \mathrm{~V}}\right.$ $=0.3)$ is indicated by a blue circle, and the SM prediction $\left(\kappa_{\lambda}=1.0, c_{2 \mathrm{~V}}=1.0\right)$ by a black star.

\section{Summary}

A search for nonresonant Higgs boson pair production $(\mathrm{HH})$ has been presented, where one of the Higgs bosons decays to a pair of bottom quarks and the other to a pair of photons. This search uses proton-proton collision data collected at $\sqrt{s}=13 \mathrm{TeV}$ by the CMS experiment at the LHC, corresponding to a total integrated luminosity of $137 \mathrm{fb}^{-1}$. No significant deviation from the background-only hypothesis is observed. Upper limits at $95 \%$ confidence level (CL) on the product of the $\mathrm{HH}$ production cross section and the branching fraction into $\gamma \gamma \mathrm{b} \overline{\mathrm{b}}$ are extracted for production in the standard model (SM) and in several scenarios beyond the SM. The expected upper limit at 95\% CL on $\sigma_{\mathrm{HH}} \mathcal{B}(\mathrm{HH} \rightarrow \gamma \gamma \mathrm{b} \overline{\mathrm{b}})$ is $0.45 \mathrm{fb}$, corresponding to about 5.2 times the SM prediction, while the observed upper limit is $0.67 \mathrm{fb}$, corresponding to 7.7 times the expected value for the SM process. The presented search has the highest sensitivity to the SM HH production to date. Upper limits at $95 \%$ $\mathrm{CL}$ on the SM HH production cross section are also derived as a function of the Higgs boson self-coupling modifier $\kappa_{\lambda} \equiv \lambda_{\mathrm{HHH}} / \lambda_{\mathrm{HHH}}^{\mathrm{SM}}$ assuming that the top quark Yukawa coupling is SM-like. The coupling modifier $\kappa_{\lambda}$ is constrained within a range $-3.3<\kappa_{\lambda}<8.5$, while the expected constraint is within a range $-2.5<\kappa_{\lambda}<8.2$ at $95 \%$ CL.

This search is combined with an analysis that targets top quark-antiquark associated production of a single Higgs boson decaying to a diphoton pair. In the scenario in which the $\mathrm{HH}$ signal has the properties predicted by the SM, the coupling modifier $\kappa_{\lambda}$ has been constrained. In addition, a simultaneous constraint on $\kappa_{\lambda}$ and the modifier of the coupling between the Higgs boson and the top quark $\kappa_{\mathrm{t}}$ is presented when both the $\mathrm{HH}$ and single Higgs boson processes are considered as signals.

Limits are also set on the cross section of nonresonant $\mathrm{HH}$ production via vector boson fusion (VBF). The most stringent limit to date is set on the product of the HH VBF 

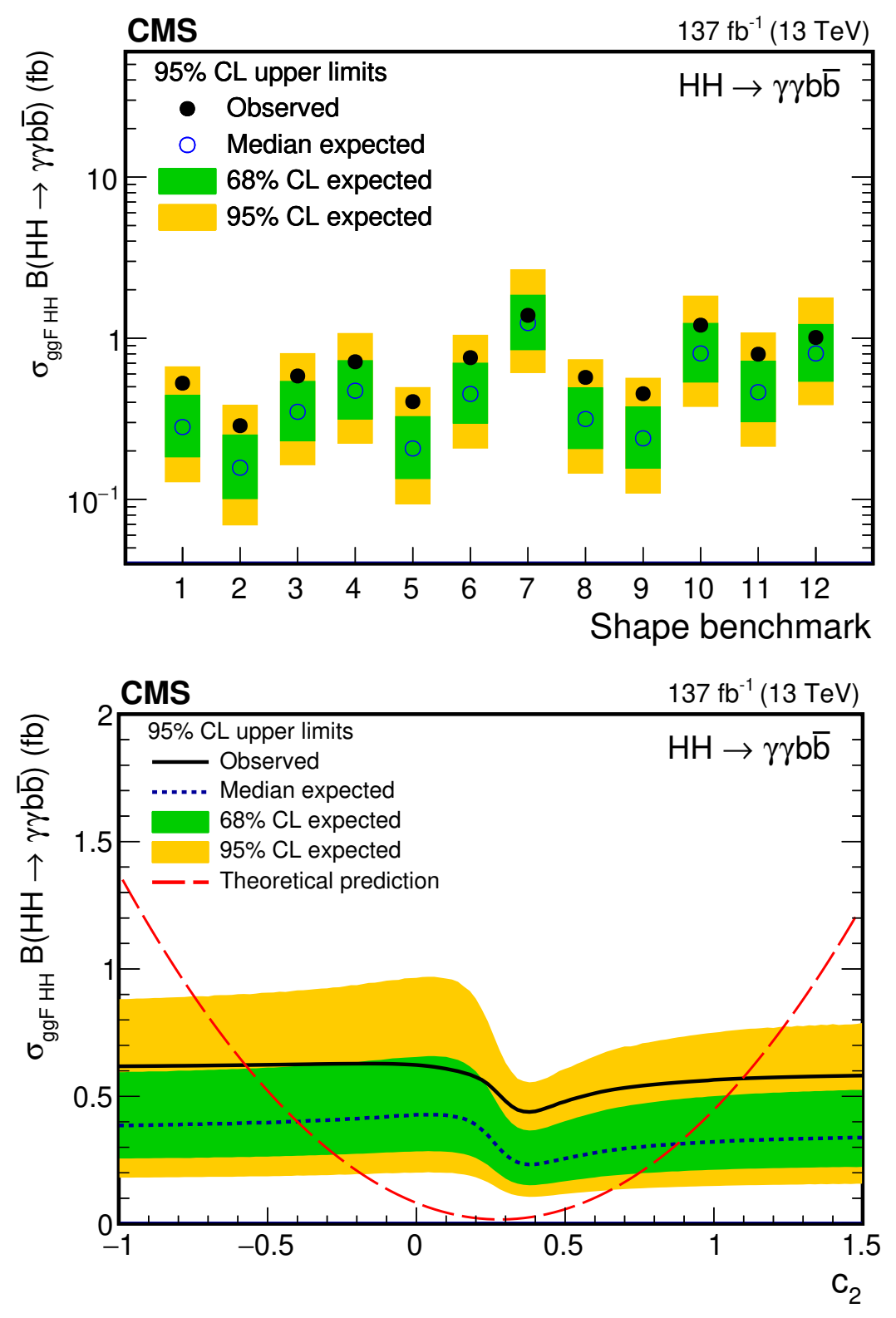

Figure 16. Expected and observed 95\% CL upper limits on the product of the ggF HH production cross section and $\mathcal{B}(\mathrm{HH} \rightarrow \gamma \gamma \mathrm{b} \overline{\mathrm{b}})$ obtained for different nonresonant benchmark models (defined in table 1) (upper) and BSM coupling $c_{2}$ (lower). In this fit, the yield of the VBF HH signal is constrained within uncertainties to the one predicted in the SM. The green and yellow bands represent, respectively, the one and two standard deviation extensions beyond the expected limit. On the lower plot the long-dashed red line shows the theoretical prediction. 
production cross section and the branching fraction into $\gamma \gamma \mathrm{b} \overline{\mathrm{b}}$. The observed (expected) upper limit at 95\% CL amounts to $1.02(0.94) \mathrm{fb}$, corresponding to 225 (208) times the SM prediction. Limits are also set as a function of the modifier of the coupling between two vector bosons and two Higgs bosons, $c_{2 \mathrm{~V}}$. The observed excluded region corresponds to $c_{2 \mathrm{~V}}<-1.3$ and $c_{2 \mathrm{~V}}>3.5$, while the expected exclusion is $c_{2 \mathrm{~V}}<-0.9$ and $c_{2 \mathrm{~V}}>3.1$.

Numerous hypotheses on coupling modifiers beyond the SM have been explored, both in the context of inclusive Higgs boson pair production and for $\mathrm{HH}$ production via gluongluon fusion and VBF. The production of Higgs boson pairs was also combined with the top quark-antiquark pair associated production of a single Higgs boson. Overall, all of the results are consistent with the SM predictions.

\section{Acknowledgments}

We congratulate our colleagues in the CERN accelerator departments for the excellent performance of the LHC and thank the technical and administrative staffs at CERN and at other CMS institutes for their contributions to the success of the CMS effort. In addition, we gratefully acknowledge the computing centers and personnel of the Worldwide LHC Computing Grid for delivering so effectively the computing infrastructure essential to our analyses. Finally, we acknowledge the enduring support for the construction and operation of the LHC and the CMS detector provided by the following funding agencies: BMBWF and FWF (Austria); FNRS and FWO (Belgium); CNPq, CAPES, FAPERJ, FAPERGS, and FAPESP (Brazil); MES (Bulgaria); CERN; CAS, MoST, and NSFC (China); COLCIENCIAS (Colombia); MSES and CSF (Croatia); RIF (Cyprus); SENESCYT (Ecuador); MoER, ERC PUT and ERDF (Estonia); Academy of Finland, MEC, and HIP (Finland); CEA and CNRS/IN2P3 (France); BMBF, DFG, and HGF (Germany); GSRT (Greece); NKFIA (Hungary); DAE and DST (India); IPM (Iran); SFI (Ireland); INFN (Italy); MSIP and NRF (Republic of Korea); MES (Latvia); LAS (Lithuania); MOE and UM (Malaysia); BUAP, CINVESTAV, CONACYT, LNS, SEP, and UASLP-FAI (Mexico); MOS (Montenegro); MBIE (New Zealand); PAEC (Pakistan); MSHE and NSC (Poland); FCT (Portugal); JINR (Dubna); MON, RosAtom, RAS, RFBR, and NRC KI (Russia); MESTD (Serbia); SEIDI, CPAN, PCTI, and FEDER (Spain); MOSTR (Sri Lanka); Swiss Funding Agencies (Switzerland); MST (Taipei); ThEPCenter, IPST, STAR, and NSTDA (Thailand); TUBITAK and TAEK (Turkey); NASU (Ukraine); STFC (United Kingdom); DOE and NSF (U.S.A.).

Individuals have received support from the Marie-Curie program and the European Research Council and Horizon 2020 Grant, contract Nos. 675440, 724704, 752730, and 765710 (European Union); the Leventis Foundation; the A.P. Sloan Foundation; the Alexander von Humboldt Foundation; the Belgian Federal Science Policy Office; the Fonds pour la Formation à la Recherche dans l'Industrie et dans l'Agriculture (FRIA-Belgium); the Agentschap voor Innovatie door Wetenschap en Technologie (IWT-Belgium); the F.R.S.-FNRS and FWO (Belgium) under the "Excellence of Science - EOS" — be.h project n. 30820817; the Beijing Municipal Science \& Technology Commission, No. Z191100007219010; the Ministry of Education, Youth and Sports (MEYS) of the Czech Republic; the Deutsche 
Forschungsgemeinschaft (DFG) under Germany's Excellence Strategy — EXC 2121 "Quantum Universe" - 390833306; the Lendület ("Momentum") Program and the János Bolyai Research Scholarship of the Hungarian Academy of Sciences, the New National Excellence Program ÚNKP, the NKFIA research grants 123842, 123959, 124845, 124850, 125105, 128713, 128786, and 129058 (Hungary); the Council of Science and Industrial Research, India; the HOMING PLUS program of the Foundation for Polish Science, cofinanced from European Union, Regional Development Fund, the Mobility Plus program of the Ministry of Science and Higher Education, the National Science Center (Poland), contracts Harmonia 2014/14/M/ST2/00428, Opus 2014/13/B/ST2/02543, 2014/15/B/ST2/03998, and 2015/19/B/ST2/02861, Sonata-bis 2012/07/E/ST2/01406; the National Priorities Research Program by Qatar National Research Fund; the Ministry of Science and Higher Education, project no. 0723-2020-0041 (Russia); the Tomsk Polytechnic University Competitiveness Enhancement Program; the Programa Estatal de Fomento de la Investigación Científica y Técnica de Excelencia María de Maeztu, grant MDM-2015-0509 and the Programa Severo Ochoa del Principado de Asturias; the Thalis and Aristeia programs cofinanced by EU-ESF and the Greek NSRF; the Rachadapisek Sompot Fund for Postdoctoral Fellowship, Chulalongkorn University and the Chulalongkorn Academic into Its 2nd Century Project Advancement Project (Thailand); the Kavli Foundation; the Nvidia Corporation; the SuperMicro Corporation; the Welch Foundation, contract C-1845; and the Weston Havens Foundation (U.S.A.).

Open Access. This article is distributed under the terms of the Creative Commons Attribution License (CC-BY 4.0), which permits any use, distribution and reproduction in any medium, provided the original author(s) and source are credited.

\section{References}

[1] ATLAS collaboration, Observation of a new particle in the search for the standard model Higgs boson with the ATLAS detector at the LHC, Phys. Lett. B 716 (2012) 1 [arXiv: 1207.7214] [INSPIRE].

[2] CMS collaboration, Observation of a new boson at a mass of $125 \mathrm{GeV}$ with the CMS experiment at the LHC, Phys. Lett. B $\mathbf{7 1 6}$ (2012) 30 [arXiv:1207.7235] [InSPIRE].

[3] CMS collaboration, Observation of a new boson with mass near $125 \mathrm{GeV}$ in pp collisions at $\sqrt{s}=7$ and $8 \mathrm{TeV}$, JHEP 06 (2013) 081 [arXiv:1303.4571] [INSPIRE].

[4] F. Englert and R. Brout, Broken symmetries and the masses of gauge bosons, Phys. Rev. Lett. 13 (1964) 321 [INSPIRE].

[5] P.W. Higgs, Broken symmetries and the masses of gauge bosons, Phys. Rev. Lett. 13 (1964) 508 [INSPIRE].

[6] M. Grazzini et al., Higgs boson pair production at NNLO with top quark mass effects, JHEP 05 (2018) 059 [arXiv: 1803. 02463] [INSPIRE].

[7] S. Dawson, S. Dittmaier and M. Spira, Neutral Higgs boson pair production at hadron colliders: QCD corrections, Phys. Rev. D 58 (1998) 115012 [hep-ph/9805244] [INSPIRE]. 
[8] S. Borowka et al., Higgs boson pair production in gluon fusion at next-to-leading order with full top-quark mass dependence, Phys. Rev. Lett. 117 (2016) 012001 [Erratum ibid. 117 (2016) 079901] [arXiv: 1604.06447] [InSPIRE].

[9] J. Baglio, F. Campanario, S. Glaus, M. Mühlleitner, M. Spira and J. Streicher, Gluon fusion into Higgs pairs at NLO QCD and the top mass scheme, Eur. Phys. J. C 79 (2019) 459 [arXiv: 1811.05692] [INSPIRE].

[10] D. de Florian and J. Mazzitelli, Higgs boson pair production at next-to-next-to-leading order in QCD, Phys. Rev. Lett. 111 (2013) 201801 [arXiv:1309.6594] [INSPIRE].

[11] D.Y. Shao, C.S. Li, H.T. Li and J. Wang, Threshold resummation effects in Higgs boson pair production at the LHC, JHEP 07 (2013) 169 [arXiv:1301.1245] [INSPIRE].

[12] D. de Florian and J. Mazzitelli, Higgs pair production at next-to-next-to-leading logarithmic accuracy at the LHC, JHEP 09 (2015) 053 [arXiv: 1505.07122] [INSPIRE].

[13] F.A. Dreyer and A. Karlberg, Vector-boson fusion Higgs pair production at $N^{3} L O$, Phys. Rev. D 98 (2018) 114016 [arXiv: 1811.07906] [InSPIRE].

[14] J. Baglio, A. Djouadi, R. Gröber, M.M. Mühlleitner, J. Quevillon and M. Spira, The measurement of the Higgs self-coupling at the LHC: theoretical status, JHEP 04 (2013) 151 [arXiv: 1212.5581] [INSPIRE].

[15] L.-S. Ling, R.-Y. Zhang, W.-G. Ma, L. Guo, W.-H. Li and X.-Z. Li, NNLO QCD corrections to Higgs pair production via vector boson fusion at hadron colliders, Phys. Rev. D 89 (2014) 073001 [arXiv:1401.7754] [INSPIRE].

[16] LHC Higgs Cross Section Working Group collaboration, Handbook of LHC Higgs cross sections: 4. Deciphering the nature of the Higgs sector, arXiv:1610.07922 [INSPIRE].

[17] F. Goertz, A. Papaefstathiou, L.L. Yang and J. Zurita, Higgs boson pair production in the $D=6$ extension of the SM, JHEP 04 (2015) 167 [arXiv: 1410.3471] [INSPIRE].

[18] CMS collaboration, Combined measurements of Higgs boson couplings in proton-proton collisions at $\sqrt{s}=13 \mathrm{TeV}$, Eur. Phys. J. C 79 (2019) 421 [arXiv:1809.10733] [InSPIRE].

[19] ATLAS collaboration, Search for Higgs boson pair production in the $\gamma \gamma b \bar{b}$ final state using pp collision data at $\sqrt{s}=8 \mathrm{TeV}$ from the ATLAS detector, Phys. Rev. Lett. 114 (2015) 081802 [arXiv: 1406.5053 ] [INSPIRE].

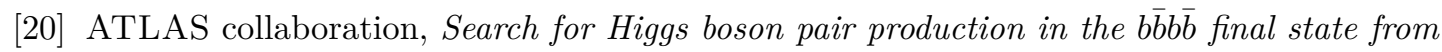
pp collisions at $\sqrt{s}=8 \mathrm{TeV}$ with the ATLAS detector, Eur. Phys. J. C $\mathbf{7 5}$ (2015) 412 [arXiv: 1506.00285] [INSPIRE].

[21] ATLAS collaboration, Searches for Higgs boson pair production in the $H H \rightarrow b b \tau \tau, \gamma \gamma W W^{*}, \gamma \gamma b b, b b b b$ channels with the ATLAS detector, Phys. Rev. D 92 (2015) 092004 [arXiv: 1509.04670] [INSPIRE].

[22] ATLAS collaboration, Search for pair production of Higgs bosons in the $b \bar{b} b \bar{b}$ final state using proton-proton collisions at $\sqrt{s}=13 \mathrm{TeV}$ with the ATLAS detector, Phys. Rev. D 94 (2016) 052002 [arXiv: 1606. 04782] [inSPIRE].

[23] ATLAS collaboration, Combination of searches for Higgs boson pairs in pp collisions at $\sqrt{s}=13 \mathrm{TeV}$ with the ATLAS detector, Phys. Lett. B 800 (2020) 135103 [arXiv: 1906.02025] [INSPIRE]. 
[24] CMS collaboration, Search for two Higgs bosons in final states containing two photons and two bottom quarks in proton-proton collisions at 8 TeV, Phys. Rev. D 94 (2016) 052012 [arXiv: 1603.06896] [INSPIRE].

[25] ATLAS collaboration, Search for Higgs boson pair production in the $\gamma \gamma b \bar{b}$ final state with $13 \mathrm{TeV}$ pp collision data collected by the ATLAS experiment, JHEP 11 (2018) 040 [arXiv: 1807.04873] [INSPIRE].

[26] CMS collaboration, Search for Higgs boson pair production in the $b \bar{b} \tau \tau$ final state in proton-proton collisions at $\sqrt{s}=8 \mathrm{TeV}$, Phys. Rev. D 96 (2017) 072004 [arXiv: 1707.00350] [INSPIRE].

[27] CMS collaboration, Search for Higgs boson pair production in events with two bottom quarks and two tau leptons in proton-proton collisions at $\sqrt{s}=13 \mathrm{TeV}$, Phys. Lett. B 778 (2018) 101 [arXiv: 1707.02909] [INSPIRE].

[28] CMS collaboration, Search for resonant and nonresonant Higgs boson pair production in the $b \bar{b} \ell \nu \ell \nu$ final state in proton-proton collisions at $\sqrt{s}=13 \mathrm{TeV}$, JHEP 01 (2018) 054 [arXiv: 1708.04188] [INSPIRE].

[29] CMS collaboration, Search for Higgs boson pair production in the $\gamma \gamma \mathrm{b} \overline{\mathrm{b}}$ final state in $p p$ collisions at $\sqrt{s}=13 \mathrm{TeV}$, Phys. Lett. B 788 (2019) 7 [arXiv:1806.00408] [InSPIRE].

[30] CMS collaboration, Combination of searches for Higgs boson pair production in proton-proton collisions at $\sqrt{s}=13 \mathrm{TeV}$, Phys. Rev. Lett. 122 (2019) 121803 [arXiv: 1811.09689] [INSPIRE].

[31] ATLAS collaboration, Search for the $H H \rightarrow b \bar{b} b \bar{b}$ process via vector-boson fusion production using proton-proton collisions at $\sqrt{s}=13 \mathrm{TeV}$ with the ATLAS detector, JHEP 07 (2020) 108 [Erratum ibid. 01 (2021) 145] [arXiv: 2001.05178] [INSPIRE].

[32] CMS collaboration, Measurements of tīH production and the CP structure of the Yukawa interaction between the Higgs boson and top quark in the diphoton decay channel, Phys. Rev. Lett. 125 (2020) 061801 [arXiv:2003.10866] [INSPIRE].

[33] F. Maltoni, D. Pagani, A. Shivaji and X. Zhao, Trilinear Higgs coupling determination via single-Higgs differential measurements at the LHC, Eur. Phys. J. C 77 (2017) 887 [arXiv: 1709.08649] [INSPIRE].

[34] G. Degrassi, P.P. Giardino, F. Maltoni and D. Pagani, Probing the Higgs self coupling via single Higgs production at the LHC, JHEP 12 (2016) 080 [arXiv:1607.04251] [INSPIRE].

[35] CMS collaboration, The CMS experiment at the CERN LHC, 2008 JINST 3 S08004 [INSPIRE].

[36] CMS collaboration, The CMS trigger system, 2017 JINST 12 P01020 [arXiv: 1609. 02366] [INSPIRE].

[37] CMS Trigger and Data Acquisition Group collaborations, The CMS high level trigger, Eur. Phys. J. C 46 (2006) 605 [hep-ex/0512077] [INSPIRE].

[38] CMS collaboration, Particle-flow reconstruction and global event description with the CMS detector, 2017 JINST 12 P10003 [arXiv:1706.04965] [INSPIRE].

[39] M. Cacciari, G.P. Salam and G. Soyez, The anti- $k_{t}$ jet clustering algorithm, JHEP 04 (2008) 063 [arXiv: 0802.1189] [INSPIRE]. 
[40] M. Cacciari, G.P. Salam and G. Soyez, FastJet user manual, Eur. Phys. J. C 72 (2012) 1896 [arXiv:1111.6097] [INSPIRE].

[41] CMS collaboration, Jet energy scale and resolution in the CMS experiment in pp collisions at $8 \mathrm{TeV}, 2017$ JINST 12 P02014 [arXiv:1607.03663] [INSPIRE].

[42] CMS collaboration, Performance of missing transverse momentum reconstruction in proton-proton collisions at $\sqrt{s}=13 \mathrm{TeV}$ using the CMS detector, 2019 JINST $14 \mathrm{P} 07004$ [arXiv: 1903.06078] [INSPIRE].

[43] G.F. Giudice, C. Grojean, A. Pomarol and R. Rattazzi, The strongly-interacting light Higgs, JHEP 06 (2007) 045 [hep-ph/0703164] [INSPIRE].

[44] A. Carvalho, M. Dall'Osso, T. Dorigo, F. Goertz, C.A. Gottardo and M. Tosi, Higgs pair production: Choosing benchmarks with cluster analysis, JHEP 04 (2016) 126 [arXiv: 1507.02245] [INSPIRE].

[45] A. Carvalho, F. Goertz, K. Mimasu, M. Gouzevitch and A. Aggarwal, On the reinterpretation of non-resonant searches for Higgs boson pairs, JHEP 02 (2021) 049 [arXiv: 1710.08261] [INSPIRE].

[46] CMS collaboration, Measurements of Higgs boson properties in the diphoton decay channel in proton-proton collisions at $\sqrt{s}=13 \mathrm{TeV}$, JHEP 11 (2018) 185 [arXiv:1804.02716] [INSPIRE].

[47] E. Bagnaschi, G. Degrassi, P. Slavich and A. Vicini, Higgs production via gluon fusion in the POWHEG approach in the SM and in the MSSM, JHEP 02 (2012) 088 [arXiv: 1111.2854$]$ [INSPIRE].

[48] G. Heinrich, S.P. Jones, M. Kerner, G. Luisoni and E. Vryonidou, NLO predictions for Higgs boson pair production with full top quark mass dependence matched to parton showers, JHEP 08 (2017) 088 [arXiv:1703.09252] [INSPIRE].

[49] G. Heinrich, S.P. Jones, M. Kerner, G. Luisoni and L. Scyboz, Probing the trilinear Higgs boson coupling in di-Higgs production at NLO QCD including parton shower effects, JHEP 06 (2019) 066 [arXiv: 1903.08137] [INSPIRE].

[50] S. Jones and S. Kuttimalai, Parton shower and NLO-matching uncertainties in Higgs boson pair production, JHEP 02 (2018) 176 [arXiv:1711.03319] [INSPIRE].

[51] G. Heinrich, S.P. Jones, M. Kerner and L. Scyboz, A non-linear EFT description of $g g \rightarrow H H$ at NLO interfaced to POWHEG, JHEP 10 (2020) 021 [arXiv:2006.16877] [INSPIRE].

[52] G. Buchalla, M. Capozi, A. Celis, G. Heinrich and L. Scyboz, Higgs boson pair production in non-linear Effective Field Theory with full $m_{t^{-}}$dependence at $N L O Q C D$, JHEP 09 (2018) 057 [arXiv: 1806. 05162] [INSPIRE].

[53] J. Alwall et al., The automated computation of tree-level and next-to-leading order differential cross sections, and their matching to parton shower simulations, JHEP $\mathbf{0 7}$ (2014) 079 [arXiv: 1405.0301] [INSPIRE].

[54] B. Hespel, D. Lopez-Val and E. Vryonidou, Higgs pair production via gluon fusion in the Two-Higgs-Doublet Model, JHEP 09 (2014) 124 [arXiv:1407.0281] [INSPIRE].

[55] R. Frederix et al., Higgs pair production at the LHC with NLO and parton-shower effects, Phys. Lett. B $\mathbf{7 3 2}$ (2014) 142 [arXiv:1401.7340] [INSPIRE]. 
[56] T. Gleisberg et al., Event generation with SHERPA 1.1, JHEP 02 (2009) 007 [arXiv:0811.4622] [INSPIRE].

[57] T. Sjöstrand et al., An introduction to PYTHIA 8.2, Comput. Phys. Commun. 191 (2015) 159 [arXiv: 1410.3012] [INSPIRE].

[58] P. Nason, A New method for combining NLO QCD with shower Monte Carlo algorithms, JHEP 11 (2004) 040 [hep-ph/0409146] [inSPIRE].

[59] S. Frixione, P. Nason and C. Oleari, Matching NLO QCD computations with Parton Shower simulations: the POWHEG method, JHEP 11 (2007) 070 [arXiv:0709.2092] [INSPIRE].

[60] S. Alioli, P. Nason, C. Oleari and E. Re, A general framework for implementing NLO calculations in shower Monte Carlo programs: the POWHEG BOX, JHEP 06 (2010) 043 [arXiv: 1002.2581] [INSPIRE].

[61] CMS collaboration, Event generator tunes obtained from underlying event and multiparton scattering measurements, Eur. Phys. J. C 76 (2016) 155 [arXiv: 1512.00815] [INSPIRE].

[62] CMS collaboration, Extraction and validation of a new set of CMS PYTHIA8 tunes from underlying-event measurements, Eur. Phys. J. C 80 (2020) 4 [arXiv:1903.12179] [INSPIRE].

[63] NNPDF collaboration, Parton distributions for the LHC Run II, JHEP 04 (2015) 040 [arXiv: 1410.8849] [INSPIRE].

[64] NNPDF collaboration, Parton distributions from high-precision collider data, Eur. Phys. J. C 77 (2017) 663 [arXiv:1706.00428] [INSPIRE].

[65] S. Carrazza, J.I. Latorre, J. Rojo and G. Watt, A compression algorithm for the combination of PDF sets, Eur. Phys. J. C 75 (2015) 474 [arXiv:1504.06469] [InSPIRE].

[66] J. Butterworth et al., PDF 4 LHC recommendations for LHC Run II, J. Phys. G 43 (2016) 023001 [arXiv: 1510.03865] [INSPIRE].

[67] S. Dulat et al., New parton distribution functions from a global analysis of quantum chromodynamics, Phys. Rev. D 93 (2016) 033006 [arXiv:1506.07443] [INSPIRE].

[68] L.A. Harland-Lang, A.D. Martin, P. Motylinski and R.S. Thorne, Parton distributions in the LHC era: MMHT 2014 PDFs, Eur. Phys. J. C 75 (2015) 204 [arXiv:1412.3989] [INSPIRE].

[69] GEANT4 collaboration, GEANT4 - a simulation toolkit, Nucl. Instrum. Meth. A $\mathbf{5 0 6}$ (2003) 250 [INSPIRE].

[70] B. Cabouat and T. Sjöstrand, Some dipole shower studies, Eur. Phys. J. C 78 (2018) 226 [arXiv: 1710.00391] [INSPIRE].

[71] B. Jäger, A. Karlberg, S. Plätzer, J. Scheller and M. Zaro, Parton-shower effects in Higgs production via vector-boson fusion, Eur. Phys. J. C 80 (2020) 756 [arXiv:2003.12435] [INSPIRE].

[72] E. Spyromitros-Xioufis, G. Tsoumakas, W. Groves and I. Vlahavas, Multi-target regression via input space expansion: treating targets as inputs, Mach. Learn. 104 (2016) 55 [arXiv: 1211.6581].

[73] CMS collaboration, Observation of the diphoton decay of the Higgs boson and measurement of its properties, Eur. Phys. J. C 74 (2014) 3076 [arXiv:1407.0558] [INSPIRE]. 
[74] CMS collaboration, The CMS phase-1 pixel detector - experience and lessons learned from two years of operation, 2019 JINST 14 C07008 [INSPIRE].

[75] CMS collaboration, Pileup mitigation at CMS in 13 TeV data, 2020 JINST 15 P09018 [arXiv:2003.00503] [INSPIRE].

[76] CMS collaboration, Performance of the DeepJet b tagging algorithm using $41.9 \mathrm{fb}^{-1}$ of data from proton-proton collisions at $13 \mathrm{TeV}$ with Phase 1 CMS detector, CMS Detector Performance Note CMS-DP-2018-058 (2018).

[77] E. Bols, J. Kieseler, M. Verzetti, M. Stoye and A. Stakia, Jet flavour classification using DeepJet, 2020 JINST 15 P12012 [arXiv:2008.10519] [INSPIRE].

[78] CMS collaboration, Determination of jet energy calibration and transverse momentum resolution in CMS, 2011 JINST 6 P11002 [arXiv:1107.4277] [INSPIRE].

[79] CMS collaboration, A deep neural network for simultaneous estimation of b jet energy and resolution, Comput. Softw. Big Sci. 4 (2020) 10 [arXiv: 1912.06046] [INSPIRE].

[80] N. Kumar and S.P. Martin, LHC search for di-Higgs decays of stoponium and other scalars in events with two photons and two bottom jets, Phys. Rev. D 90 (2014) 055007 [arXiv:1404.0996] [INSPIRE].

[81] S. Hochreiter and J. Schmidhuber, Long short-term memory, Neural Comput. 9 (1997) 1735 [INSPIRE].

[82] T.Q. Nguyen et al., Topology classification with deep learning to improve real-time event selection at the LHC, Comput. Softw. Big Sci. 3 (2019) 12 [arXiv:1807.00083] [INSPIRE].

[83] F. Chollet et al., Keras, https://github.com/fchollet/keras (2015).

[84] M. Abadi et al., TensorFlow: Large-scale machine learning on heterogeneous systems, (2015), Software available from http://tensorflow.org/.

[85] J.C. Collins and D.E. Soper, Angular distribution of dileptons in high-energy hadron collisions, Phys. Rev. D 16 (1977) 2219 [InSPIRE].

[86] T. Chen and C. Guestrin, XGBoost: A scalable tree boosting system, in Proceedings of the 22nd ACM SIGKDD International Conference on Knowledge Discovery and Data Mining, KDD 785, ACM, New York, NY, U.S.A. (2016) [arXiv:1603.02754] [INSPIRE].

[87] T. Hastie, R. Tibshirani and J. Friedman, The elements of statistical learning, Springer-Verlag New York, 2nd edition (2009) [DOI].

[88] CMS collaboration, Performance of quark/gluon discrimination in 8 TeV pp data, Tech. Rep. CMS-PAS-JME-13-002 (2013).

[89] CMS collaboration, Jet algorithms performance in 13 TeV data, Tech. Rep. CMS-PAS-JME-16-003 (2017).

[90] CMS collaboration, Search for direct production of supersymmetric partners of the top quark in the all-jets final state in proton-proton collisions at $\sqrt{s}=13 \mathrm{TeV}$, JHEP 10 (2017) 005 [arXiv:1707.03316] [INSPIRE].

[91] M.J. Oreglia, A study of the reactions $\psi^{\prime} \rightarrow \gamma \gamma \psi$, Ph.D. Thesis, Stanford University (1980), SLAC Report SLAC-R-236 [http://www.slac.stanford.edu/cgi-wrap/getdoc/slac-r-236.pdf].

[92] P.D. Dauncey, M. Kenzie, N. Wardle and G.J. Davies, Handling uncertainties in background shapes: the discrete profiling method, 2015 JINST 10 P04015 [arXiv:1408.6865] [INSPIRE]. 
[93] CMS collaboration, Performance of photon reconstruction and identification with the CMS detector in proton-proton collisions at $\sqrt{s}=8 \mathrm{TeV}, 2015$ JINST $10 \mathrm{P} 08010$ [arXiv: 1502.02702] [INSPIRE].

[94] CMS collaboration, Identification of heavy-flavour jets with the CMS detector in pp collisions at $13 \mathrm{TeV}, 2018$ JINST 13 P05011 [arXiv:1712.07158] [INSPIRE].

[95] CMS collaboration, Measurement of the inclusive $W$ and $Z$ production cross sections in $p p$ collisions at $\sqrt{s}=7 \mathrm{TeV}$, JHEP 10 (2011) 132 [arXiv:1107.4789] [INSPIRE].

[96] CMS collaboration, CMS luminosity measurements for the 2016 data taking period, Tech. Rep. CMS-PAS-LUM-17-001 (2017).

[97] CMS collaboration, CMS luminosity measurement for the 2017 data-taking period at $\sqrt{s}=13 \mathrm{TeV}$, Tech. Rep. CMS-PAS-LUM-17-004 (2018).

[98] CMS collaboration, CMS luminosity measurement for the 2018 data-taking period at $\sqrt{s}=13 \mathrm{TeV}$, Tech. Rep. CMS-PAS-LUM-18-002 (2019).

[99] T. Junk, Confidence level computation for combining searches with small statistics, Nucl. Instrum. Meth. A 434 (1999) 435 [hep-ex/9902006] [InSPIRE].

[100] A.L. Read, Presentation of search results: The $C L_{s}$ technique, J. Phys. G 28 (2002) 2693 [INSPIRE].

[101] G. Cowan, K. Cranmer, E. Gross and O. Vitells, Asymptotic formulae for likelihood-based tests of new physics, Eur. Phys. J. C 71 (2011) 1554 [Erratum ibid. 73 (2013) 2501] [arXiv: 1007.1727] [INSPIRE].

[102] ATLAS and CMS collaborations, LHC Higgs Combination Group, Procedure for the LHC Higgs boson search combination in Summer 2011, Tech. Rep. CMS-NOTE-2011-005; ATL-PHYS-PUB-2011-11 (2011).

[103] CMS collaboration, A measurement of the Higgs boson mass in the diphoton decay channel, Phys. Lett. B 805 (2020) 135425 [arXiv: 2002.06398] [INSPIRE].

[104] CMS collaboration, Measurement of the Higgs boson production rate in association with top quarks in final states with electrons, muons, and hadronically decaying tau leptons at $\sqrt{s}=13 \mathrm{TeV}$, submitted to Eur. Phys. J. C [arXiv:2011.03652] [InSPIRE]. 


\section{The CMS collaboration}

Yerevan Physics Institute, Yerevan, Armenia

A.M. Sirunyan ${ }^{\dagger}$, A. Tumasyan

Institut für Hochenergiephysik, Wien, Austria

W. Adam, T. Bergauer, M. Dragicevic, A. Escalante Del Valle, R. Frühwirth ${ }^{1}$, M. Jeitler ${ }^{1}$, N. Krammer, L. Lechner, D. Liko, I. Mikulec, F.M. Pitters, J. Schieck ${ }^{1}$, R. Schöfbeck, M. Spanring, S. Templ, W. Waltenberger, C.-E. Wulz ${ }^{1}$, M. Zarucki

Institute for Nuclear Problems, Minsk, Belarus

V. Chekhovsky, A. Litomin, V. Makarenko

Universiteit Antwerpen, Antwerpen, Belgium

M.R. Darwish ${ }^{2}$, E.A. De Wolf, X. Janssen, T. Kello ${ }^{3}$, A. Lelek, H. Rejeb Sfar, P. Van Mechelen, S. Van Putte, N. Van Remortel

Vrije Universiteit Brussel, Brussel, Belgium

F. Blekman, E.S. Bols, J. D'Hondt, J. De Clercq, S. Lowette, S. Moortgat, A. Morton, D. Müller, A.R. Sahasransu, S. Tavernier, W. Van Doninck, P. Van Mulders

\section{Université Libre de Bruxelles, Bruxelles, Belgium}

D. Beghin, B. Bilin, B. Clerbaux, G. De Lentdecker, B. Dorney, L. Favart, A. Grebenyuk, A.K. Kalsi, K. Lee, I. Makarenko, L. Moureaux, L. Pétré, A. Popov, N. Postiau, E. Starling, L. Thomas, C. Vander Velde, P. Vanlaer, D. Vannerom, L. Wezenbeek

\section{Ghent University, Ghent, Belgium}

T. Cornelis, D. Dobur, M. Gruchala, I. Khvastunov ${ }^{4}$, G. Mestdach, M. Niedziela, C. Roskas, K. Skovpen, M. Tytgat, W. Verbeke, B. Vermassen, M. Vit

\section{Université Catholique de Louvain, Louvain-la-Neuve, Belgium}

A. Bethani, G. Bruno, F. Bury, C. Caputo, P. David, C. Delaere, M. Delcourt, I.S. Donertas, A. Giammanco, V. Lemaitre, K. Mondal, J. Prisciandaro, A. Taliercio, M. Teklishyn, P. Vischia, S. Wertz, S. Wuyckens

Centro Brasileiro de Pesquisas Fisicas, Rio de Janeiro, Brazil

G.A. Alves, C. Hensel, A. Moraes

Universidade do Estado do Rio de Janeiro, Rio de Janeiro, Brazil

W.L. Aldá Júnior, E. Belchior Batista Das Chagas, H. BRANDAO MALBOUISSON, W. Carvalho, J. Chinellato ${ }^{5}$, E. Coelho, E.M. Da Costa, G.G. Da Silveira ${ }^{6}$, D. De Jesus Damiao, S. Fonseca De Souza, J. Martins ${ }^{7}$, D. Matos Figueiredo, C. Mora Herrera, L. Mundim, H. Nogima, P. Rebello Teles, L.J. Sanchez Rosas, A. Santoro, S.M. Silva Do Amaral, A. Sznajder, M. Thiel, F. Torres Da Silva De Araujo, A. Vilela Pereira 
Universidade Estadual Paulista ${ }^{a}$, Universidade Federal do $\mathrm{ABC}^{b}$, São Paulo, Brazil

C.A. Bernardes ${ }^{a, a}$, L. Calligaris ${ }^{a}$, T.R. Fernandez Perez Tomei $^{a}$, E.M. Gregores ${ }^{a, b}$, D.S. Lemos ${ }^{a}$, P.G. Mercadante ${ }^{a, b}$, S.F. Novaes ${ }^{a}$, Sandra S. Padula ${ }^{a}$

Institute for Nuclear Research and Nuclear Energy, Bulgarian Academy of Sciences, Sofia, Bulgaria

A. Aleksandrov, G. Antchev, I. Atanasov, R. Hadjiiska, P. Iaydjiev, M. Misheva, M. Rodozov, M. Shopova, G. Sultanov

University of Sofia, Sofia, Bulgaria

A. Dimitrov, T. Ivanov, L. Litov, B. Pavlov, P. Petkov, A. Petrov

Beihang University, Beijing, China

T. Cheng, W. Fang ${ }^{3}$, Q. Guo, M. Mittal, H. Wang, L. Yuan

Department of Physics, Tsinghua University, Beijing, China

M. Ahmad, G. Bauer, Z. Hu, Y. Wang, K. Yi ${ }^{8,9}$

Institute of High Energy Physics, Beijing, China

E. Chapon, G.M. Chen ${ }^{10}$, H.S. Chen ${ }^{10}$, M. Chen, T. Javaid ${ }^{10}$, A. Kapoor, D. Leggat, H. Liao, Z.-A. LIU ${ }^{10}$, R. Sharma, A. Spiezia, J. Tao, J. Thomas-wilsker, J. Wang, H. Zhang, S. Zhang ${ }^{10}$, J. Zhao

State Key Laboratory of Nuclear Physics and Technology, Peking University, Beijing, China

A. Agapitos, Y. Ban, C. Chen, Q. Huang, A. Levin, Q. Li, M. Lu, X. Lyu, Y. Mao, S.J. Qian, D. Wang, Q. Wang, J. Xiao

Sun Yat-Sen University, Guangzhou, China

Z. You

Institute of Modern Physics and Key Laboratory of Nuclear Physics and Ionbeam Application (MOE) - Fudan University, Shanghai, China

X. $\mathrm{Gao}^{3}$, H. Okawa

Zhejiang University, Hangzhou, China

M. Xiao

Universidad de Los Andes, Bogota, Colombia

C. Avila, A. Cabrera, C. Florez, J. Fraga, A. Sarkar, M.A. Segura Delgado

Universidad de Antioquia, Medellin, Colombia

J. Jaramillo, J. Mejia Guisao, F. Ramirez, J.D. Ruiz Alvarez, C.A. Salazar González, N. Vanegas Arbelaez

University of Split, Faculty of Electrical Engineering, Mechanical Engineering and Naval Architecture, Split, Croatia

D. Giljanovic, N. Godinovic, D. Lelas, I. Puljak 
University of Split, Faculty of Science, Split, Croatia

Z. Antunovic, M. Kovac, T. Sculac

Institute Rudjer Boskovic, Zagreb, Croatia

V. Brigljevic, B.K. Chitroda, D. Ferencek, D. Majumder, M. Roguljic, A. Starodumov ${ }^{11}$, T. Susa

University of Cyprus, Nicosia, Cyprus

M.W. Ather, A. Attikis, E. Erodotou, A. Ioannou, G. Kole, M. Kolosova, S. Konstantinou, J. Mousa, C. Nicolaou, F. Ptochos, P.A. Razis, H. Rykaczewski, H. Saka, D. Tsiakkouri

Charles University, Prague, Czech Republic

M. Finger ${ }^{12}$, M. Finger Jr. ${ }^{12}$, A. Kveton, J. Tomsa

Escuela Politecnica Nacional, Quito, Ecuador

E. Ayala

Universidad San Francisco de Quito, Quito, Ecuador

E. Carrera Jarrin

Academy of Scientific Research and Technology of the Arab Republic of Egypt, Egyptian Network of High Energy Physics, Cairo, Egypt

H. Abdalla ${ }^{13}$, A.A. Abdelalim ${ }^{14,15}$, Y. Assran ${ }^{16,17}$

Center for High Energy Physics (CHEP-FU), Fayoum University, El-Fayoum, Egypt

A. Lotfy, M.A. Mahmoud

National Institute of Chemical Physics and Biophysics, Tallinn, Estonia

S. Bhowmik, A. Carvalho Antunes De Oliveira, R.K. Dewanjee, K. Ehataht, M. Kadastik, J. Pata, M. Raidal, C. Veelken

Department of Physics, University of Helsinki, Helsinki, Finland

P. Eerola, L. Forthomme, H. Kirschenmann, K. Osterberg, M. Voutilainen

Helsinki Institute of Physics, Helsinki, Finland

E. Brücken, F. Garcia, J. Havukainen, V. Karimäki, M.S. Kim, R. Kinnunen, T. Lampén, K. Lassila-Perini, S. Lehti, T. Lindén, H. Siikonen, E. Tuominen, J. Tuominiemi

\section{Lappeenranta University of Technology, Lappeenranta, Finland}

P. Luukka, T. Tuuva

IRFU, CEA, Université Paris-Saclay, Gif-sur-Yvette, France

C. Amendola, M. Besancon, F. Couderc, M. Dejardin, D. Denegri, J.L. Faure, F. Ferri, S. Ganjour, A. Givernaud, P. Gras, G. Hamel de Monchenault, P. Jarry, B. Lenzi, E. Locci, J. Malcles, J. Rander, A. Rosowsky, M.Ö. Sahin, A. Savoy-Navarro ${ }^{18}$, M. Titov, G.B. Yu

Laboratoire Leprince-Ringuet, CNRS/IN2P3, Ecole Polytechnique, Institut Polytechnique de Paris, Palaiseau, France

S. Ahuja, F. Beaudette, M. Bonanomi, A. Buchot Perraguin, P. Busson, C. Charlot, O. Davignon, B. Diab, G. Falmagne, R. Granier de Cassagnac, A. Hakimi, I. Kucher, 
A. Lobanov, C. Martin Perez, M. Nguyen, C. Ochando, P. Paganini, J. Rembser, R. Salerno, J.B. Sauvan, Y. Sirois, A. Zabi, A. Zghiche

Université de Strasbourg, CNRS, IPHC UMR 7178, Strasbourg, France J.-L. Agram ${ }^{19}$, J. Andrea, D. Apparu, D. Bloch, G. Bourgatte, J.-M. Brom, E.C. Chabert, C. Collard, D. Darej, J.-C. Fontaine ${ }^{19}$, U. Goerlach, C. Grimault, A.-C. Le Bihan, P. Van Hove

Université de Lyon, Université Claude Bernard Lyon 1, CNRS-IN2P3, Institut de Physique Nucléaire de Lyon, Villeurbanne, France

E. Asilar, S. Beauceron, C. Bernet, G. Boudoul, C. Camen, A. Carle, N. Chanon, D. Contardo, P. Depasse, H. El Mamouni, J. Fay, S. Gascon, M. Gouzevitch, B. Ille, Sa. Jain, I.B. Laktineh, H. Lattaud, A. Lesauvage, M. Lethuillier, L. Mirabito, K. Shchablo,

L. Torterotot, G. Touquet, M. Vander Donckt, S. Viret

Georgian Technical University, Tbilisi, Georgia

I. Bagaturia $^{20}$, Z. Tsamalaidze ${ }^{12}$

RWTH Aachen University, I. Physikalisches Institut, Aachen, Germany

L. Feld, K. Klein, M. Lipinski, D. Meuser, A. Pauls, M.P. Rauch, J. Schulz, M. Teroerde RWTH Aachen University, III. Physikalisches Institut A, Aachen, Germany

D. Eliseev, M. Erdmann, P. Fackeldey, B. Fischer, S. Ghosh, T. Hebbeker, K. Hoepfner, H. Keller, L. Mastrolorenzo, M. Merschmeyer, A. Meyer, G. Mocellin, S. Mondal, S. Mukherjee, D. Noll, A. Novak, T. Pook, A. Pozdnyakov, Y. Rath, H. Reithler, J. Roemer, A. Schmidt, S.C. Schuler, A. Sharma, S. Wiedenbeck, S. Zaleski

RWTH Aachen University, III. Physikalisches Institut B, Aachen, Germany

C. Dziwok, G. Flügge, W. Haj Ahmad ${ }^{21}$, O. Hlushchenko, T. Kress, A. Nowack, C. Pistone, O. Pooth, D. Roy, H. Sert, A. Stahl ${ }^{22}$, T. Ziemons

\section{Deutsches Elektronen-Synchrotron, Hamburg, Germany}

H. Aarup Petersen, M. Aldaya Martin, P. Asmuss, I. Babounikau, S. Baxter, O. Behnke, A. Bermúdez Martínez, A.A. Bin Anuar, K. Borras ${ }^{23}$, V. Botta, D. Brunner, A. Campbell, A. Cardini, P. Connor, S. Consuegra Rodríguez, V. Danilov, M.M. Defranchis, L. Didukh, D. Domínguez Damiani, G. Eckerlin, D. Eckstein, L.I. Estevez Banos, E. Gallo ${ }^{24}$, A. Geiser, A. Giraldi, A. Grohsjean, M. Guthoff, A. Harb, A. Jafari ${ }^{25}$, N.Z. Jomhari, H. Jung, A. Kasem ${ }^{23}$, M. Kasemann, H. Kaveh, C. Kleinwort, J. Knolle, D. Krücker, W. Lange, T. Lenz, J. Lidrych, K. Lipka, W. Lohmann ${ }^{26}$, T. Madlener, R. Mankel, I.-A. MelzerPellmann, J. Metwally, A.B. Meyer, M. Meyer, J. Mnich, A. Mussgiller, V. Myronenko, Y. Otarid, D. Pérez Adán, S.K. Pflitsch, D. Pitzl, A. Raspereza, A. Saggio, A. Saibel, M. Savitskyi, V. Scheurer, C. Schwanenberger, A. Singh, R.E. Sosa Ricardo, N. Tonon, O. Turkot, A. Vagnerini, M. Van De Klundert, R. Walsh, D. Walter, Y. Wen, K. Wichmann,

C. Wissing, S. Wuchterl, O. Zenaiev, R. Zlebcik

\section{University of Hamburg, Hamburg, Germany}

R. Aggleton, S. Bein, L. Benato, A. Benecke, K. De Leo, T. Dreyer, M. Eich, F. Feindt, A. Fröhlich, C. Garbers, E. Garutti, P. Gunnellini, J. Haller, A. Hinzmann, A. Karavdina, 
G. Kasieczka, R. Klanner, R. Kogler, V. Kutzner, J. Lange, T. Lange, A. Malara, C.E.N. Niemeyer, A. Nigamova, K.J. Pena Rodriguez, O. Rieger, P. Schleper, M. Schröder, J. Schwandt, D. Schwarz, J. Sonneveld, H. Stadie, G. Steinbrück, A. Tews, B. Vormwald, I. Zoi

Karlsruher Institut fuer Technologie, Karlsruhe, Germany

J. Bechtel, T. Berger, E. Butz, R. Caspart, T. Chwalek, W. De Boer, A. Dierlamm, A. Droll, K. El Morabit, N. Faltermann, K. Flöh, M. Giffels, J.o. Gosewisch, A. Gottmann, F. Hartmann ${ }^{22}$, C. Heidecker, U. Husemann, I. Katkov ${ }^{27}$, P. Keicher, R. Koppenhöfer, S. Maier, M. Metzler, S. Mitra, Th. Müller, M. Musich, M. Neukum, G. Quast, K. Rabbertz, J. Rauser, D. Savoiu, D. Schäfer, M. Schnepf, D. Seith, I. Shvetsov, H.J. Simonis, R. Ulrich, J. Van Der Linden, R.F. Von Cube, M. Wassmer, M. Weber, S. Wieland, R. Wolf, S. Wozniewski, S. Wunsch

Institute of Nuclear and Particle Physics (INPP), NCSR Demokritos, Aghia Paraskevi, Greece

G. Anagnostou, P. Asenov, G. Daskalakis, T. Geralis, A. Kyriakis, D. Loukas, G. Paspalaki, A. Stakia

\section{National and Kapodistrian University of Athens, Athens, Greece}

M. Diamantopoulou, D. Karasavvas, G. Karathanasis, P. Kontaxakis, C.K. Koraka, A. Manousakis-katsikakis, A. Panagiotou, I. Papavergou, N. Saoulidou, K. Theofilatos, E. Tziaferi, K. Vellidis, E. Vourliotis

National Technical University of Athens, Athens, Greece

G. Bakas, K. Kousouris, I. Papakrivopoulos, G. Tsipolitis, A. Zacharopoulou

University of Ioánnina, Ioánnina, Greece

I. Evangelou, C. Foudas, P. Gianneios, P. Katsoulis, P. Kokkas, N. Manthos, I. Papadopoulos, J. Strologas

MTA-ELTE Lendület CMS Particle and Nuclear Physics Group, Eötvös Loránd University, Budapest, Hungary

M. Csanad, M.M.A. Gadallah ${ }^{28}$, S. Lökös ${ }^{29}$, P. Major, K. Mandal, A. Mehta, G. Pasztor, O. Surányi, G.I. Veres

Wigner Research Centre for Physics, Budapest, Hungary

M. Bartók ${ }^{30}$, G. Bencze, C. Hajdu, D. Horvath ${ }^{31}$, F. Sikler, V. Veszpremi, G. Vesztergombi ${ }^{\dagger}$

Institute of Nuclear Research ATOMKI, Debrecen, Hungary

S. Czellar, J. Karancsi ${ }^{30}$, J. Molnar, Z. Szillasi, D. Teyssier

Institute of Physics, University of Debrecen, Debrecen, Hungary

P. Raics, Z.L. Trocsanyi ${ }^{32}$, B. Ujvari

Eszterhazy Karoly University, Karoly Robert Campus, Gyongyos, Hungary

T. Csorgo ${ }^{33}$, F. Nemes ${ }^{33}$, T. Novak 
Indian Institute of Science (IISc), Bangalore, India

S. Choudhury, J.R. Komaragiri, D. Kumar, L. Panwar, P.C. Tiwari

National Institute of Science Education and Research, HBNI, Bhubaneswar, India

S. Bahinipati ${ }^{34}$, D. Dash, C. Kar, P. Mal, T. Mishra, V.K. Muraleedharan Nair Bindhu ${ }^{35}$, A. Nayak ${ }^{35}$, N. Sur, S.K. Swain

Panjab University, Chandigarh, India

S. Bansal, S.B. Beri, V. Bhatnagar, G. Chaudhary, S. Chauhan, N. Dhingra ${ }^{36}$, R. Gupta, A. Kaur, S. Kaur, P. Kumari, M. Meena, K. Sandeep, J.B. Singh, A.K. Virdi

University of Delhi, Delhi, India

A. Ahmed, A. Bhardwaj, B.C. Choudhary, R.B. Garg, M. Gola, S. Keshri, A. Kumar, M. Naimuddin, P. Priyanka, K. Ranjan, A. Shah

Saha Institute of Nuclear Physics, HBNI, Kolkata, India

M. Bharti ${ }^{37}$, R. Bhattacharya, S. Bhattacharya, D. Bhowmik, S. Dutta, S. Ghosh, B. Gomber ${ }^{38}$, M. Maity ${ }^{39}$, S. Nandan, P. Palit, P.K. Rout, G. Saha, B. Sahu, S. Sarkar, M. Sharan, B. Singh ${ }^{37}$, S. Thakur ${ }^{37}$

Indian Institute of Technology Madras, Madras, India

P.K. Behera, S.C. Behera, P. Kalbhor, A. Muhammad, R. Pradhan, P.R. Pujahari, A. Sharma, A.K. Sikdar

Bhabha Atomic Research Centre, Mumbai, India

D. Dutta, V. Jha, V. Kumar, D.K. Mishra, K. Naskar ${ }^{40}$, P.K. Netrakanti, L.M. Pant, P. Shukla

Tata Institute of Fundamental Research-A, Mumbai, India

T. Aziz, S. Dugad, G.B. Mohanty, U. Sarkar

Tata Institute of Fundamental Research-B, Mumbai, India

S. Banerjee, S. Bhattacharya, S. Chatterjee, R. Chudasama, M. Guchait, S. Karmakar, S. Kumar, G. Majumder, K. Mazumdar, S. Mukherjee, D. Roy

Indian Institute of Science Education and Research (IISER), Pune, India

S. Dube, B. Kansal, S. Pandey, A. Rane, A. Rastogi, S. Sharma

Department of Physics, Isfahan University of Technology, Isfahan, Iran

H. Bakhshiansohi ${ }^{41}$, M. Zeinali ${ }^{42}$

Institute for Research in Fundamental Sciences (IPM), Tehran, Iran

S. Chenarani ${ }^{43}$, S.M. Etesami, M. Khakzad, M. Mohammadi Najafabadi

University College Dublin, Dublin, Ireland

M. Felcini, M. Grunewald 
INFN Sezione di Bari ${ }^{a}$, Università di Bari ${ }^{b}$, Politecnico di Bari ${ }^{c}$, Bari, Italy

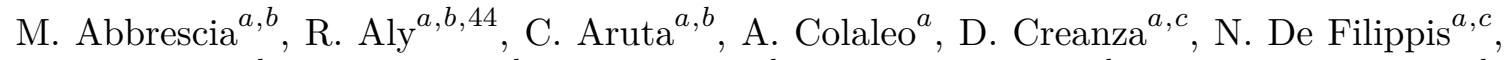
M. De Palma ${ }^{a, b}$, A. Di Florio ${ }^{a, b}$, A. Di Pilato ${ }^{a, b}$, W. Elmetenawee ${ }^{a, b}{\text {, L. } \text { Fiore }^{a} \text {, A. Gelmi }}^{a, b}$,

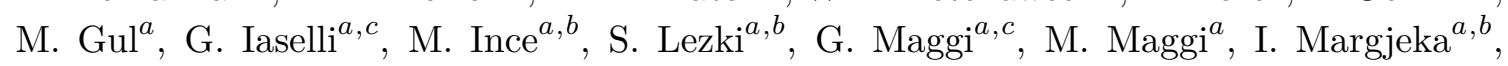
V. Mastrapasqua ${ }^{a, b}$, J.A. Merlin ${ }^{a}$, S. My ${ }^{a, b}$, S. Nuzzo ${ }^{a, b}$, A. Pompili ${ }^{a, b}$, G. Pugliese ${ }^{a, c}$,

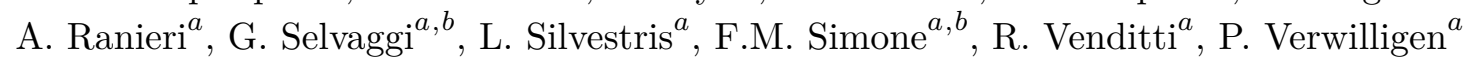

INFN Sezione di Bologna ${ }^{a}$, Università di Bologna ${ }^{b}$, Bologna, Italy

G. Abbiendi ${ }^{a}$, C. Battilana ${ }^{a, b}$, D. Bonacorsi ${ }^{a, b}$, L. Borgonovi $^{a}$, S. Braibant-Giacomelli ${ }^{a, b}$, R. Campanini ${ }^{a, b}$, P. Capiluppi ${ }^{a, b}$, A. Castro $^{a, b}$, F.R. Cavallo ${ }^{a}$, C. Ciocca $^{a}$, M. Cuffiani $^{a, b}$, G.M. Dallavalle $^{a}$, T. Diotalevi ${ }^{a, b}$, F. Fabbri $^{a}$, A. Fanfani ${ }^{a, b}$, E. Fontanesi $^{a, b}$, P. Giacomelli $^{a}$, L. Giommi ${ }^{a, b}$, C. Grandi ${ }^{a}$, L. Guiducci ${ }^{a, b}$, F. Iemmi ${ }^{a, b}$, S. Lo Meo ${ }^{a, 45}$, S. Marcellini ${ }^{a}$, G. Masetti ${ }^{a}$, F.L. Navarria ${ }^{a, b}$, A. Perrotta ${ }^{a}$, F. Primavera ${ }^{a, b}$, A.M. Rossi ${ }^{a, b}$, T. Rovelli ${ }^{a, b}$, G.P. Siroli ${ }^{a, b}$, N. Tosi $^{a}$

INFN Sezione di Catania ${ }^{a}$, Università di Catania ${ }^{b}$, Catania, Italy

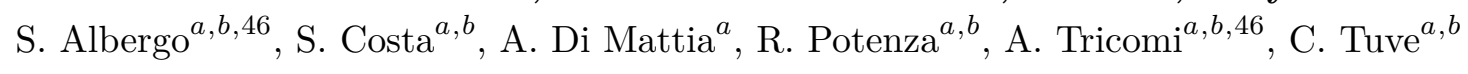

INFN Sezione di Firenze ${ }^{a}$, Università di Firenze ${ }^{b}$, Firenze, Italy

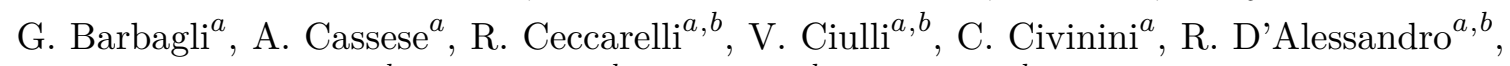
F. Fiori ${ }^{a}$, E. Focardi $^{a, b}$, G. Latino $^{a, b}$, P. Lenzi $^{a, b}$, M. Lizzo $^{a, b}$, M. Meschini $^{a}$, S. Paoletti $^{a}$, R. Seidita ${ }^{a, b}$, G. Sguazzoni ${ }^{a}$, L. Viliani ${ }^{a}$

INFN Laboratori Nazionali di Frascati, Frascati, Italy

L. Benussi, S. Bianco, D. Piccolo

INFN Sezione di Genova ${ }^{a}$, Università di Genova ${ }^{b}$, Genova, Italy

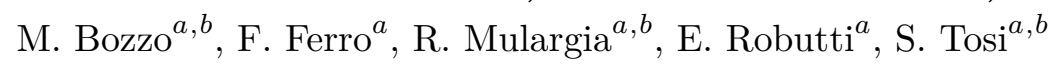

INFN Sezione di Milano-Bicocca ${ }^{a}$, Università di Milano-Bicocca ${ }^{b}$, Milano, Italy

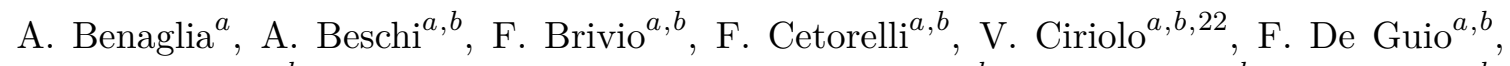
M.E. Dinardo ${ }^{a, b}$, P. Dini ${ }^{a}$, S. Gennai ${ }^{a}$, A. Ghezzi ${ }^{a, b}$, P. Govoni ${ }^{a, b}$, L. Guzzi ${ }^{a, b}$, M. Malberti ${ }^{a}$, S. Malvezzi ${ }^{a}$, A. Massironi ${ }^{a}$, D. Menasce ${ }^{a}$, F. Monti ${ }^{a, b}$, L. Moroni ${ }^{a}$, M. Paganoni ${ }^{a, b}$, D. Pedrini ${ }^{a}$, S. Ragazzi ${ }^{a, b}$, T. Tabarelli de Fatis ${ }^{a, b}$, D. Valsecchi ${ }^{a, b, 22}$, D. Zuolo ${ }^{a, b}$

INFN Sezione di Napoli ${ }^{a}$, Università di Napoli 'Federico II' ${ }^{b}$, Napoli, Italy, Università della Basilicata ${ }^{c}$, Potenza, Italy, Università G. Marconi ${ }^{d}$, Roma, Italy

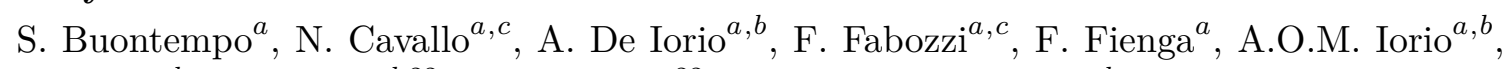

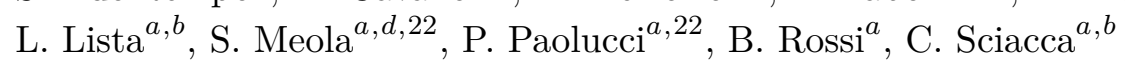

INFN Sezione di Padova ${ }^{a}$, Università di Padova ${ }^{b}$, Padova, Italy, Università di Trento ${ }^{c}$, Trento, Italy

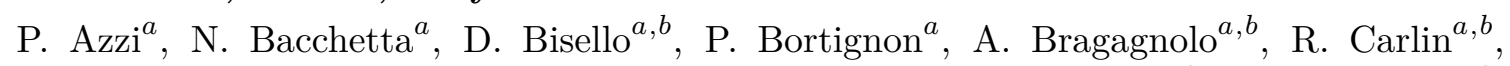

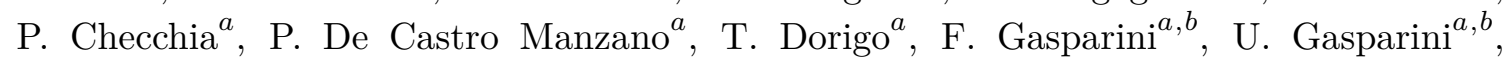




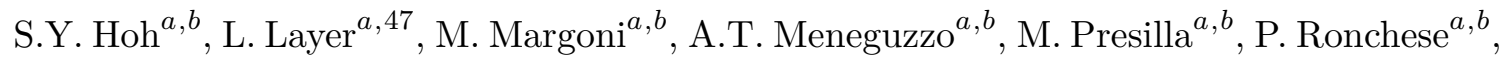

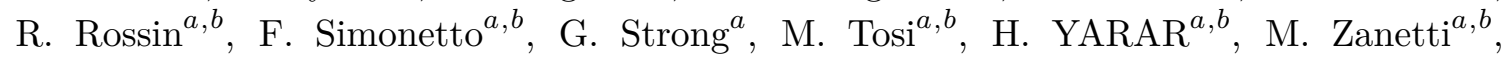
P. Zotto ${ }^{a, b}$, A. Zucchetta ${ }^{a, b}$, G. Zumerle ${ }^{a, b}$

INFN Sezione di Pavia ${ }^{a}$, Università di Pavia ${ }^{b}$, Pavia, Italy

C. Aime ${ }^{a, b}$, A. Braghieri ${ }^{a}$, S. Calzaferri ${ }^{a, b}$, D. Fiorina ${ }^{a, b}$, P. Montagna ${ }^{a, b}$, S.P. Ratti ${ }^{a, b}$,

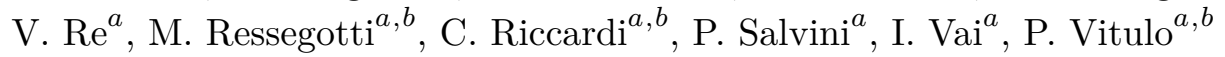

INFN Sezione di Perugia ${ }^{a}$, Università di Perugia ${ }^{b}$, Perugia, Italy

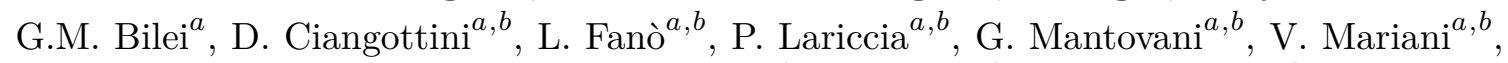
M. Menichelli ${ }^{a}$, F. Moscatelli ${ }^{a}$, A. Piccinelli $^{a, b}$, A. Rossi $^{a, b}$, A. Santocchia $^{a, b}$, D. Spiga $^{a}$, T. Tedeschi ${ }^{a, b}$

INFN Sezione di Pisa ${ }^{a}$, Università di Pisa ${ }^{b}$, Scuola Normale Superiore di Pisa ${ }^{c}$, Pisa Italy, Università di Siena ${ }^{d}$, Siena, Italy

K. Androsov ${ }^{a}$, P. Azzurri ${ }^{a}$, G. Bagliesi ${ }^{a}$, V. Bertacchi ${ }^{a, c}$, L. Bianchini ${ }^{a}$, T. Boccali $^{a}$, E. Bossini, R. Castaldi ${ }^{a}$, M.A. Ciocci ${ }^{a, b}$, R. Dell'Orso ${ }^{a}$, M.R. Di Domenico $^{a, b}$, S. Donato $^{a}{ }^{,}$ A. Giassi ${ }^{a}$, M.T. Grippo ${ }^{a}$, F. Ligabue ${ }^{a, c}$, E. Manca ${ }^{a, c}$, G. Mandorli ${ }^{a, c}$, A. Messineo ${ }^{a, b}$, F. Palla ${ }^{a}$, G. Ramirez-Sanchez ${ }^{a, c}$, A. Rizzi ${ }^{a, b}$, G. Rolandi ${ }^{a, c}$, S. Roy Chowdhury ${ }^{a, c}$, A. Scribano ${ }^{a}$, N. Shafiei ${ }^{a, b}$, P. Spagnolo ${ }^{a}$, R. Tenchini ${ }^{a}$, G. Tonelli ${ }^{a, b}$, N. Turini ${ }^{a}$, A. Venturi ${ }^{a}$, P.G. Verdini ${ }^{a}$

INFN Sezione di Roma ${ }^{a}$, Sapienza Università di Roma ${ }^{b}$, Rome, Italy F. Cavallari ${ }^{a}$, M. Cipriani ${ }^{a, b}$, D. Del Re ${ }^{a, b}$, E. Di Marco ${ }^{a}$, M. $\operatorname{Diemoz}^{a}$, E. Longo $^{a, b}$, P. Meridiani ${ }^{a}$, G. Organtini ${ }^{a, b}$, F. Pandolfi ${ }^{a}$, R. Paramatti ${ }^{a, b}$, C. Quaranta ${ }^{a, b}$,

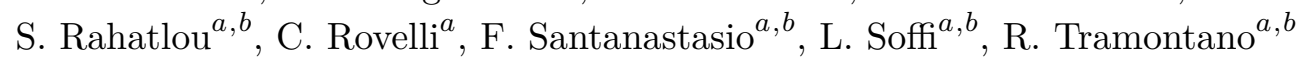

INFN Sezione di Torino ${ }^{a}$, Università di Torino ${ }^{b}$, Torino, Italy, Università del Piemonte Orientale ${ }^{c}$, Novara, Italy

N. Amapane ${ }^{a, b}$, R. Arcidiacono ${ }^{a, c}$, S. Argiro ${ }^{a, b}$, M. Arneodo ${ }^{a, c}$, N. Bartosik $^{a}$, R. Bellan ${ }^{a, b}$, A. Bellora ${ }^{a, b}$, J. Berenguer Antequera ${ }^{a, b}$, C. Biino ${ }^{a}$, A. Cappati ${ }^{a, b}$, N. Cartiglia ${ }^{a}$, S. Cometti $^{a}$, M. Costa ${ }^{a, b}$, R. Covarelli ${ }^{a, b}$, N. Demaria ${ }^{a}$, B. Kiani ${ }^{a, b}$,

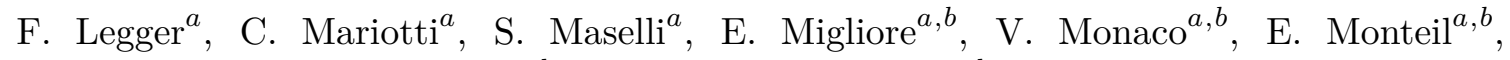
M. Monteno ${ }^{a}$, M.M. Obertino ${ }^{a, b}$, G. Ortona ${ }^{a}$, L. Pacher $^{a}, b$, N. Pastrone $^{a}$, M. Pelliccioni $^{a}$, G.L. Pinna Angioni ${ }^{a, b}$, M. Ruspa ${ }^{a, c}$, R. Salvatico ${ }^{a, b}$, F. Siviero $^{a, b}$, V. Sola ${ }^{a}$, A. Solano ${ }^{a, b}$, D. Soldi ${ }^{a, b}$, A. Staiano ${ }^{a}$, M. Tornago ${ }^{a, b}$, D. Trocino ${ }^{a, b}$

INFN Sezione di Trieste ${ }^{a}$, Università di Trieste ${ }^{b}$, Trieste, Italy

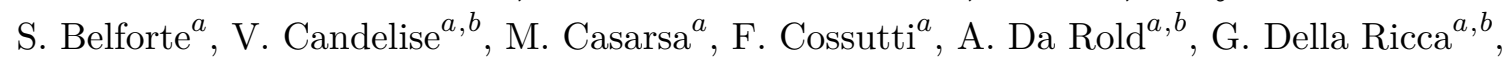
F. Vazzoler ${ }^{a, b}$

Kyungpook National University, Daegu, Korea

S. Dogra, C. Huh, B. Kim, D.H. Kim, G.N. Kim, J. Lee, S.W. Lee, C.S. Moon, Y.D. Oh, S.I. Pak, B.C. Radburn-Smith, S. Sekmen, Y.C. Yang

Chonnam National University, Institute for Universe and Elementary Particles, Kwangju, Korea

H. Kim, D.H. Moon 
Hanyang University, Seoul, Korea

B. Francois, T.J. Kim, J. Park

Korea University, Seoul, Korea

S. Cho, S. Choi, Y. Go, B. Hong, K. Lee, K.S. Lee, J. Lim, J. Park, S.K. Park, J. Yoo

Kyung Hee University, Department of Physics, Seoul, Republic of Korea

J. Goh, A. Gurtu

Sejong University, Seoul, Korea

H.S. Kim, Y. Kim

Seoul National University, Seoul, Korea

J. Almond, J.H. Bhyun, J. Choi, S. Jeon, J. Kim, J.S. Kim, S. Ko, H. Kwon, H. Lee, S. Lee,

K. Nam, B.H. Oh, M. Oh, S.B. Oh, H. Seo, U.K. Yang, I. Yoon

University of Seoul, Seoul, Korea

D. Jeon, J.H. Kim, B. Ko, J.S.H. Lee, I.C. Park, Y. Roh, D. Song, I.J. Watson

Yonsei University, Department of Physics, Seoul, Korea

S. Ha, H.D. Yoo

Sungkyunkwan University, Suwon, Korea

Y. Choi, C. Hwang, Y. Jeong, H. Lee, Y. Lee, I. Yu

College of Engineering and Technology, American University of the Middle East (AUM), Kuwait

Y. Maghrbi

Riga Technical University, Riga, Latvia

V. Veckalns ${ }^{48}$

Vilnius University, Vilnius, Lithuania

M. Ambrozas, A. Juodagalvis, A. Rinkevicius, G. Tamulaitis, A. Vaitkevicius

National Centre for Particle Physics, Universiti Malaya, Kuala Lumpur, Malaysia

W.A.T. Wan Abdullah, M.N. Yusli, Z. Zolkapli

Universidad de Sonora (UNISON), Hermosillo, Mexico

J.F. Benitez, A. Castaneda Hernandez, J.A. Murillo Quijada, L. Valencia Palomo

Centro de Investigacion y de Estudios Avanzados del IPN, Mexico City, Mexico

G. Ayala, H. Castilla-Valdez, E. De La Cruz-Burelo, I. Heredia-De La Cruz ${ }^{49}$, R. Lopez-

Fernandez, C.A. Mondragon Herrera, D.A. Perez Navarro, A. Sanchez-Hernandez

Universidad Iberoamericana, Mexico City, Mexico

S. Carrillo Moreno, C. Oropeza Barrera, M. Ramirez-Garcia, F. Vazquez Valencia

Benemerita Universidad Autonoma de Puebla, Puebla, Mexico

I. Pedraza, H.A. Salazar Ibarguen, C. Uribe Estrada 
University of Montenegro, Podgorica, Montenegro

J. Mijuskovic ${ }^{4}$, N. Raicevic

University of Auckland, Auckland, New Zealand

D. Krofcheck

University of Canterbury, Christchurch, New Zealand

S. Bheesette, P.H. Butler

National Centre for Physics, Quaid-I-Azam University, Islamabad, Pakistan

A. Ahmad, M.I. Asghar, A. Awais, M.I.M. Awan, H.R. Hoorani, W.A. Khan, M.A. Shah, M. Shoaib, M. Waqas

AGH University of Science and Technology Faculty of Computer Science, Electronics and Telecommunications, Krakow, Poland

V. Avati, L. Grzanka, M. Malawski

National Centre for Nuclear Research, Swierk, Poland

H. Bialkowska, M. Bluj, B. Boimska, T. Frueboes, M. Górski, M. Kazana, M. Szleper, P. Traczyk, P. Zalewski

Institute of Experimental Physics, Faculty of Physics, University of Warsaw, Warsaw, Poland

K. Bunkowski, K. Doroba, A. Kalinowski, M. Konecki, J. Krolikowski, M. Walczak

Laboratório de Instrumentação e Física Experimental de Partículas, Lisboa, Portugal

M. Araujo, P. Bargassa, D. Bastos, A. Boletti, P. Faccioli, M. Gallinaro, J. Hollar, N. Leonardo, T. Niknejad, J. Seixas, K. Shchelina, O. Toldaiev, J. Varela

Joint Institute for Nuclear Research, Dubna, Russia

S. Afanasiev, D. Budkouski, P. Bunin, M. Gavrilenko, I. Golutvin, I. Gorbunov,

A. Kamenev, V. Karjavine, A. Lanev, A. Malakhov, V. Matveev ${ }^{50,51}$, V. Palichik,

V. Perelygin, M. Savina, D. Seitova, V. Shalaev, S. Shmatov, S. Shulha, V. Smirnov,

O. Teryaev, N. Voytishin, A. Zarubin, I. Zhizhin

Petersburg Nuclear Physics Institute, Gatchina (St. Petersburg), Russia

G. Gavrilov, V. Golovtcov, Y. Ivanov, V. Kim ${ }^{52}$, E. Kuznetsova ${ }^{53}$, V. Murzin, V. Oreshkin, I. Smirnov, D. Sosnov, V. Sulimov, L. Uvarov, S. Volkov, A. Vorobyev

Institute for Nuclear Research, Moscow, Russia

Yu. Andreev, A. Dermenev, S. Gninenko, N. Golubev, A. Karneyeu, M. Kirsanov, N. Krasnikov, A. Pashenkov, G. Pivovarov, D. Tlisov ${ }^{\dagger}$, A. Toropin

Institute for Theoretical and Experimental Physics named by A.I. Alikhanov of NRC 'Kurchatov Institute', Moscow, Russia

V. Epshteyn, V. Gavrilov, N. Lychkovskaya, A. Nikitenko ${ }^{54}$, V. Popov, G. Safronov, A. Spiridonov, A. Stepennov, M. Toms, E. Vlasov, A. Zhokin 
Moscow Institute of Physics and Technology, Moscow, Russia

T. Aushev

National Research Nuclear University 'Moscow Engineering Physics Institute' (MEPhI), Moscow, Russia

R. Chistov ${ }^{55}$, M. Danilov ${ }^{55}$, A. Oskin, P. Parygin, S. Polikarpov ${ }^{55}$

P.N. Lebedev Physical Institute, Moscow, Russia

V. Andreev, M. Azarkin, I. Dremin, M. Kirakosyan, A. Terkulov

Skobeltsyn Institute of Nuclear Physics, Lomonosov Moscow State University, Moscow, Russia

A. Belyaev, E. Boos, V. Bunichev, M. Dubinin ${ }^{56}$, L. Dudko, A. Gribushin, V. Klyukhin,

O. Kodolova, I. Lokhtin, S. Obraztsov, M. Perfilov, S. Petrushanko, V. Savrin

Novosibirsk State University (NSU), Novosibirsk, Russia

V. Blinov ${ }^{57}$, T. Dimova ${ }^{57}$, L. Kardapoltsev ${ }^{57}$, I. Ovtin ${ }^{57}$, Y. Skovpen ${ }^{57}$, S. Zakharov ${ }^{57}$

Institute for High Energy Physics of National Research Centre 'Kurchatov Institute', Protvino, Russia

I. Azhgirey, I. Bayshev, V. Kachanov, A. Kalinin, D. Konstantinov, V. Petrov, R. Ryutin, A. Sobol, S. Troshin, N. Tyurin, A. Uzunian, A. Volkov

National Research Tomsk Polytechnic University, Tomsk, Russia

A. Babaev, A. Iuzhakov, V. Okhotnikov, L. Sukhikh

Tomsk State University, Tomsk, Russia

V. Borchsh, V. Ivanchenko, E. Tcherniaev

University of Belgrade: Faculty of Physics and VINCA Institute of Nuclear Sciences, Belgrade, Serbia

P. Adzic ${ }^{58}$, M. Dordevic, P. Milenovic, J. Milosevic

Centro de Investigaciones Energéticas Medioambientales y Tecnológicas (CIEMAT), Madrid, Spain

M. Aguilar-Benitez, J. Alcaraz Maestre, A. Álvarez Fernández, I. Bachiller, M. Barrio Luna, Cristina F. Bedoya, C.A. Carrillo Montoya, M. Cepeda, M. Cerrada, N. Colino, B. De La Cruz, A. Delgado Peris, J.P. Fernández Ramos, J. Flix, M.C. Fouz, O. Gonzalez Lopez, S. Goy Lopez, J.M. Hernandez, M.I. Josa, J. León Holgado, D. Moran, Á. Navarro Tobar, A. Pérez-Calero Yzquierdo, J. Puerta Pelayo, I. Redondo, L. Romero, S. Sánchez Navas, M.S. Soares, L. Urda Gómez, C. Willmott

Universidad Autónoma de Madrid, Madrid, Spain

C. Albajar, J.F. de Trocóniz, R. Reyes-Almanza

Universidad de Oviedo, Instituto Universitario de Ciencias y Tecnologías Espaciales de Asturias (ICTEA), Oviedo, Spain

B. Alvarez Gonzalez, J. Cuevas, C. Erice, J. Fernandez Menendez, S. Folgueras, I. Gonzalez Caballero, E. Palencia Cortezon, C. Ramón Álvarez, J. Ripoll Sau, V. Rodríguez Bouza, 
A. Trapote

Instituto de Física de Cantabria (IFCA), CSIC-Universidad de Cantabria, Santander, Spain

J.A. Brochero Cifuentes, I.J. Cabrillo, A. Calderon, B. Chazin Quero, J. Duarte Campderros, M. Fernandez, C. Fernandez Madrazo, P.J. Fernández Manteca, A. García Alonso, G. Gomez, C. Martinez Rivero, P. Martinez Ruiz del Arbol, F. Matorras, J. Piedra Gomez, C. Prieels, F. Ricci-Tam, T. Rodrigo, A. Ruiz-Jimeno, L. Scodellaro, N. Trevisani, I. Vila, J.M. Vizan Garcia

\section{University of Colombo, Colombo, Sri Lanka}

MK Jayananda, B. Kailasapathy ${ }^{59}$, D.U.J. Sonnadara, DDC Wickramarathna

University of Ruhuna, Department of Physics, Matara, Sri Lanka

W.G.D. Dharmaratna, K. Liyanage, N. Perera, N. Wickramage

\section{CERN, European Organization for Nuclear Research, Geneva, Switzerland}

T.K. Aarrestad, D. Abbaneo, E. Auffray, G. Auzinger, J. Baechler, P. Baillon, A.H. Ball, D. Barney, J. Bendavid, N. Beni, M. Bianco, A. Bocci, E. Brondolin, T. Camporesi, M. Capeans Garrido, G. Cerminara, S.S. Chhibra, L. Cristella, D. d'Enterria, A. Dabrowski, N. Daci, A. David, A. De Roeck, M. Deile, R. Di Maria, M. Dobson, M. Dünser, N. Dupont, A. Elliott-Peisert, N. Emriskova, F. Fallavollita ${ }^{60}$, D. Fasanella, S. Fiorendi, A. Florent, G. Franzoni, J. Fulcher, W. Funk, S. Giani, D. Gigi, K. Gill, F. Glege, L. Gouskos, M. Haranko, J. Hegeman, Y. Iiyama, V. Innocente, T. James, P. Janot, J. Kaspar, J. Kieseler, M. Komm, N. Kratochwil, C. Lange, S. Laurila, P. Lecoq, K. Long, C. Lourenço, L. Malgeri, S. Mallios, M. Mannelli, F. Meijers, S. Mersi, E. Meschi, F. Moortgat, M. Mulders, S. Orfanelli, L. Orsini, F. Pantaleo ${ }^{22}$, L. Pape, E. Perez, M. Peruzzi, A. Petrilli, G. Petrucciani, A. Pfeiffer, M. Pierini, M. Pitt, T. Quast, D. Rabady, A. Racz, M. Rieger, M. Rovere, H. Sakulin, J. Salfeld-Nebgen, S. Scarfi, C. Schäfer, C. Schwick, M. Selvaggi, A. Sharma, P. Silva, W. Snoeys, P. Sphicas ${ }^{61}$, S. Summers, V.R. Tavolaro, D. Treille, A. Tsirou, G.P. Van Onsem, M. Verzetti, K.A. Wozniak, W.D. Zeuner

\section{Paul Scherrer Institut, Villigen, Switzerland}

L. Caminada ${ }^{62}$, A. Ebrahimi, W. Erdmann, R. Horisberger, Q. Ingram, H.C. Kaestli, D. Kotlinski, U. Langenegger, M. Missiroli, T. Rohe

ETH Zurich - Institute for Particle Physics and Astrophysics (IPA), Zurich, Switzerland

M. Backhaus, P. Berger, A. Calandri, N. Chernyavskaya, A. De Cosa, G. Dissertori, M. Dittmar, M. Donegà, C. Dorfer, T. Gadek, T.A. Gómez Espinosa, C. Grab, D. Hits, W. Lustermann, A.-M. Lyon, R.A. Manzoni, M.T. Meinhard, F. Micheli, F. Nessi-Tedaldi, J. Niedziela, F. Pauss, V. Perovic, G. Perrin, S. Pigazzini, M.G. Ratti, M. Reichmann, C. Reissel, T. Reitenspiess, B. Ristic, D. Ruini, D.A. Sanz Becerra, M. Schönenberger, V. Stampf, J. Steggemann ${ }^{63}$, R. Wallny, D.H. Zhu 
Universität Zürich, Zurich, Switzerland

C. Amsler ${ }^{64}$, C. Botta, D. Brzhechko, M.F. Canelli, A. De Wit, R. Del Burgo, J.K. Heikkilä, M. Huwiler, A. Jofrehei, B. Kilminster, S. Leontsinis, A. Macchiolo, P. Meiring, V.M. Mikuni, U. Molinatti, I. Neutelings, G. Rauco, A. Reimers, P. Robmann, S. Sanchez Cruz, K. Schweiger, Y. Takahashi

National Central University, Chung-Li, Taiwan

C. Adloff ${ }^{65}$, C.M. Kuo, W. Lin, A. Roy, T. Sarkar ${ }^{39}$, S.S. Yu

National Taiwan University (NTU), Taipei, Taiwan

L. Ceard, P. Chang, Y. Chao, K.F. Chen, P.H. Chen, W.-S. Hou, Y.y. Li, R.-S. Lu,

E. Paganis, A. Psallidas, A. Steen, E. Yazgan, P.r. Yu

Chulalongkorn University, Faculty of Science, Department of Physics, Bangkok, Thailand

B. Asavapibhop, C. Asawatangtrakuldee, N. Srimanobhas

Çukurova University, Physics Department, Science and Art Faculty, Adana, Turkey

F. Boran, S. Damarseckin ${ }^{66}$, Z.S. Demiroglu, F. Dolek, C. Dozen ${ }^{67}$, I. Dumanoglu ${ }^{68}$, E. Eskut, G. Gokbulut, Y. Guler, E. Gurpinar Guler ${ }^{69}$, I. $\operatorname{Hos}^{70}$, C. Isik, E.E. Kangal ${ }^{71}$, O. Kara, A. Kayis Topaksu, U. Kiminsu, G. Onengut, K. Ozdemir ${ }^{72}$, A. Polatoz, A.E. Simsek, B. Tali ${ }^{73}$, U.G. Tok, S. Turkcapar, I.S. Zorbakir, C. Zorbilmez

Middle East Technical University, Physics Department, Ankara, Turkey B. Isildak ${ }^{74}$, G. Karapinar ${ }^{75}$, K. Ocalan ${ }^{76}$, M. Yalvac ${ }^{77}$

Bogazici University, Istanbul, Turkey

B. Akgun, I.O. Atakisi, E. Gülmez, M. Kaya ${ }^{78}$, O. Kaya ${ }^{79}$, Ö. Özçelik, S. Tekten ${ }^{80}$, E.A. Yetkin ${ }^{81}$

Istanbul Technical University, Istanbul, Turkey

A. Cakir, K. Cankocak ${ }^{68}$, Y. Komurcu, S. Sen ${ }^{82}$

Istanbul University, Istanbul, Turkey

F. Aydogmus Sen, S. Cerci ${ }^{73}$, B. Kaynak, S. Ozkorucuklu, D. Sunar Cerci ${ }^{73}$

Institute for Scintillation Materials of National Academy of Science of Ukraine, Kharkov, Ukraine

B. Grynyov

National Scientific Center, Kharkov Institute of Physics and Technology, Kharkov, Ukraine

L. Levchuk

University of Bristol, Bristol, United Kingdom

E. Bhal, S. Bologna, J.J. Brooke, A. Bundock, E. Clement, D. Cussans, H. Flacher, J. Goldstein, G.P. Heath, H.F. Heath, L. Kreczko, B. Krikler, S. Paramesvaran, T. Sakuma, S. Seif El Nasr-Storey, V.J. Smith, N. Stylianou ${ }^{83}$, J. Taylor, A. Titterton 


\section{Rutherford Appleton Laboratory, Didcot, United Kingdom}

K.W. Bell, A. Belyaev ${ }^{84}$, C. Brew, R.M. Brown, D.J.A. Cockerill, K.V. Ellis, K. Harder, S. Harper, J. Linacre, K. Manolopoulos, D.M. Newbold, E. Olaiya, D. Petyt, T. Reis, T. Schuh, C.H. Shepherd-Themistocleous, A. Thea, I.R. Tomalin, T. Williams

\section{Imperial College, London, United Kingdom}

R. Bainbridge, P. Bloch, S. Bonomally, J. Borg, S. Breeze, O. Buchmuller, V. Cepaitis, G.S. Chahal ${ }^{85}$, D. Colling, P. Dauncey, G. Davies, M. Della Negra, G. Fedi, G. Hall, M.H. Hassanshahi, G. Iles, J. Langford, L. Lyons, A.-M. Magnan, S. Malik, A. Martelli, V. Milosevic, J. Nash ${ }^{86}$, V. Palladino, M. Pesaresi, D.M. Raymond, A. Richards, A. Rose, E. Scott, C. Seez, A. Shtipliyski, A. Tapper, K. Uchida, T. Virdee ${ }^{22}$, N. Wardle, S.N. Webb, D. Winterbottom, A.G. Zecchinelli

\section{Brunel University, Uxbridge, United Kingdom}

J.E. Cole, A. Khan, P. Kyberd, C.K. Mackay, I.D. Reid, L. Teodorescu, S. Zahid

\section{Baylor University, Waco, U.S.A.}

S. Abdullin, A. Brinkerhoff, B. Caraway, J. Dittmann, K. Hatakeyama, A.R. Kanuganti, B. McMaster, N. Pastika, S. Sawant, C. Smith, C. Sutantawibul, J. Wilson

Catholic University of America, Washington, DC, U.S.A.

R. Bartek, A. Dominguez, R. Uniyal, A.M. Vargas Hernandez

The University of Alabama, Tuscaloosa, U.S.A.

A. Buccilli, O. Charaf, S.I. Cooper, D. Di Croce, S.V. Gleyzer, C. Henderson, C.U. Perez, P. Rumerio, C. West

\section{Boston University, Boston, U.S.A.}

A. Akpinar, A. Albert, D. Arcaro, C. Cosby, Z. Demiragli, D. Gastler, J. Rohlf, K. Salyer, D. Sperka, D. Spitzbart, I. Suarez, S. Yuan, D. Zou

\section{Brown University, Providence, U.S.A.}

G. Benelli, B. Burkle, X. Coubez ${ }^{23}$, D. Cutts, Y.t. Duh, M. Hadley, U. Heintz, J.M. $\operatorname{Hogan}^{87}$, K.H.M. Kwok, E. Laird, G. Landsberg, K.T. Lau, J. Lee, J. Luo, M. Narain, S. Sagir ${ }^{88}$, E. Usai, W.Y. Wong, X. Yan, D. Yu, W. Zhang

\section{University of California, Davis, Davis, U.S.A.}

R. Band, C. Brainerd, R. Breedon, M. Calderon De La Barca Sanchez, M. Chertok, J. Conway, R. Conway, P.T. Cox, R. Erbacher, C. Flores, F. Jensen, O. Kukral, R. Lander, M. Mulhearn, D. Pellett, M. Shi, D. Taylor, M. Tripathi, Y. Yao, F. Zhang

\section{University of California, Los Angeles, U.S.A.}

M. Bachtis, R. Cousins, A. Dasgupta, A. Datta, D. Hamilton, J. Hauser, M. Ignatenko, M.A. Iqbal, T. Lam, N. Mccoll, W.A. Nash, S. Regnard, D. Saltzberg, C. Schnaible, B. Stone, V. Valuev

University of California, Riverside, Riverside, U.S.A.

K. Burt, Y. Chen, R. Clare, J.W. Gary, G. Hanson, G. Karapostoli, O.R. Long, N. Manganelli, M. Olmedo Negrete, W. Si, S. Wimpenny, Y. Zhang 
University of California, San Diego, La Jolla, U.S.A.

J.G. Branson, P. Chang, S. Cittolin, S. Cooperstein, N. Deelen, J. Duarte, R. Gerosa, L. Giannini, D. Gilbert, V. Krutelyov, J. Letts, M. Masciovecchio, S. May, S. Padhi, M. Pieri, V. Sharma, M. Tadel, A. Vartak, F. Würthwein, A. Yagil

University of California, Santa Barbara - Department of Physics, Santa Barbara, U.S.A.

N. Amin, C. Campagnari, M. Citron, A. Dorsett, V. Dutta, J. Incandela, M. Kilpatrick, B. Marsh, H. Mei, A. Ovcharova, H. Qu, M. Quinnan, J. Richman, U. Sarica, D. Stuart, S. Wang

California Institute of Technology, Pasadena, U.S.A.

A. Bornheim, O. Cerri, I. Dutta, J.M. Lawhorn, N. Lu, J. Mao, H.B. Newman, J. Ngadiuba, T.Q. Nguyen, M. Spiropulu, J.R. Vlimant, C. Wang, S. Xie, Z. Zhang, R.Y. Zhu

Carnegie Mellon University, Pittsburgh, U.S.A.

J. Alison, M.B. Andrews, T. Ferguson, T. Mudholkar, M. Paulini, I. Vorobiev

University of Colorado Boulder, Boulder, U.S.A.

J.P. Cumalat, W.T. Ford, E. MacDonald, R. Patel, A. Perloff, K. Stenson, K.A. Ulmer, S.R. Wagner

Cornell University, Ithaca, U.S.A.

J. Alexander, Y. Cheng, J. Chu, D.J. Cranshaw, K. Mcdermott, J. Monroy, J.R. Patterson, D. Quach, A. Ryd, W. Sun, S.M. Tan, Z. Tao, J. Thom, P. Wittich, M. Zientek

Fermi National Accelerator Laboratory, Batavia, U.S.A.

M. Albrow, M. Alyari, G. Apollinari, A. Apresyan, A. Apyan, S. Banerjee, L.A.T. Bauerdick, A. Beretvas, D. Berry, J. Berryhill, P.C. Bhat, K. Burkett, J.N. Butler, A. Canepa, G.B. Cerati, H.W.K. Cheung, F. Chlebana, M. Cremonesi, K.F. Di Petrillo, V.D. Elvira, J. Freeman, Z. Gecse, L. Gray, D. Green, S. Grünendahl, O. Gutsche, R.M. Harris, R. Heller, T.C. Herwig, J. Hirschauer, B. Jayatilaka, S. Jindariani, M. Johnson, U. Joshi, P. Klabbers, T. Klijnsma, B. Klima, M.J. Kortelainen, S. Lammel, D. Lincoln, R. Lipton, T. Liu, J. Lykken, C. Madrid, K. Maeshima, C. Mantilla, D. Mason, P. McBride, P. Merkel, S. Mrenna, S. Nahn, V. O'Dell, V. Papadimitriou, K. Pedro, C. Pena ${ }^{56}$, O. Prokofyev, F. Ravera, A. Reinsvold Hall, L. Ristori, B. Schneider, E. Sexton-Kennedy, N. Smith, A. Soha, L. Spiegel, S. Stoynev, J. Strait, L. Taylor, S. Tkaczyk, N.V. Tran, L. Uplegger, E.W. Vaandering, H.A. Weber

University of Florida, Gainesville, U.S.A.

D. Acosta, P. Avery, D. Bourilkov, L. Cadamuro, V. Cherepanov, F. Errico, R.D. Field, D. Guerrero, B.M. Joshi, M. Kim, J. Konigsberg, A. Korytov, K.H. Lo, K. Matchev, N. Menendez, G. Mitselmakher, D. Rosenzweig, K. Shi, J. Sturdy, J. Wang, E. Yigitbasi, X. Zuo

Florida State University, Tallahassee, U.S.A.

T. Adams, A. Askew, D. Diaz, R. Habibullah, S. Hagopian, V. Hagopian, K.F. Johnson, R. Khurana, T. Kolberg, G. Martinez, H. Prosper, C. Schiber, R. Yohay, J. Zhang 
Florida Institute of Technology, Melbourne, U.S.A.

M.M. Baarmand, S. Butalla, T. Elkafrawy ${ }^{89}$, M. Hohlmann, R. Kumar Verma, D. Noonan, M. Rahmani, M. Saunders, F. Yumiceva

University of Illinois at Chicago (UIC), Chicago, U.S.A.

M.R. Adams, L. Apanasevich, H. Becerril Gonzalez, R. Cavanaugh, X. Chen, S. Dittmer, O. Evdokimov, C.E. Gerber, D.A. Hangal, D.J. Hofman, C. Mills, G. Oh, T. Roy, M.B. Tonjes, N. Varelas, J. Viinikainen, X. Wang, Z. Wu, Z. Ye

The University of Iowa, Iowa City, U.S.A.

M. Alhusseini, K. Dilsiz ${ }^{90}$, S. Durgut, R.P. Gandrajula, M. Haytmyradov, V. Khristenko, O.K. Köseyan, J.-P. Merlo, A. Mestvirishvili ${ }^{91}$, A. Moeller, J. Nachtman, H. Ogul ${ }^{92}$, Y. Onel, F. Ozok ${ }^{93}$, A. Penzo, C. Snyder, E. $\operatorname{Tiras}^{94}$, J. Wetzel

Johns Hopkins University, Baltimore, U.S.A.

O. Amram, B. Blumenfeld, L. Corcodilos, M. Eminizer, A.V. Gritsan, S. Kyriacou, P. Maksimovic, J. Roskes, M. Swartz, T.Á. Vámi

The University of Kansas, Lawrence, U.S.A.

C. Baldenegro Barrera, P. Baringer, A. Bean, A. Bylinkin, T. Isidori, S. Khalil, J. King,

G. Krintiras, A. Kropivnitskaya, C. Lindsey, N. Minafra, M. Murray, C. Rogan, C. Royon, S. Sanders, E. Schmitz, J.D. Tapia Takaki, Q. Wang, J. Williams, G. Wilson

Kansas State University, Manhattan, U.S.A.

S. Duric, A. Ivanov, K. Kaadze, D. Kim, Y. Maravin, T. Mitchell, A. Modak

Lawrence Livermore National Laboratory, Livermore, U.S.A.

F. Rebassoo, D. Wright

University of Maryland, College Park, U.S.A.

E. Adams, A. Baden, O. Baron, A. Belloni, S.C. Eno, Y. Feng, N.J. Hadley, S. Jabeen, R.G. Kellogg, T. Koeth, A.C. Mignerey, S. Nabili, M. Seidel, A. Skuja, S.C. Tonwar, L. Wang, K. Wong

Massachusetts Institute of Technology, Cambridge, U.S.A.

D. Abercrombie, R. Bi, S. Brandt, W. Busza, I.A. Cali, Y. Chen, M. D'Alfonso, G. Gomez Ceballos, M. Goncharov, P. Harris, M. Hu, M. Klute, D. Kovalskyi, J. Krupa, Y.-J. Lee, P.D. Luckey, B. Maier, A.C. Marini, C. Mironov, X. Niu, C. Paus, D. Rankin, C. Roland, G. Roland, Z. Shi, G.S.F. Stephans, K. Tatar, D. Velicanu, J. Wang, T.W. Wang, Z. Wang, B. Wyslouch

University of Minnesota, Minneapolis, U.S.A.

R.M. Chatterjee, A. Evans, P. Hansen, J. Hiltbrand, Sh. Jain, M. Krohn, Y. Kubota, Z. Lesko, J. Mans, M. Revering, R. Rusack, R. Saradhy, N. Schroeder, N. Strobbe, M.A. Wadud

University of Mississippi, Oxford, U.S.A.

J.G. Acosta, S. Oliveros 
University of Nebraska-Lincoln, Lincoln, U.S.A.

K. Bloom, M. Bryson, S. Chauhan, D.R. Claes, C. Fangmeier, L. Finco, F. Golf, J.R. González Fernández, C. Joo, I. Kravchenko, J.E. Siado, G.R. Snow ${ }^{\dagger}$, W. Tabb, F. Yan

State University of New York at Buffalo, Buffalo, U.S.A.

G. Agarwal, H. Bandyopadhyay, L. Hay, I. Iashvili, A. Kharchilava, C. McLean, D. Nguyen, J. Pekkanen, S. Rappoccio

Northeastern University, Boston, U.S.A.

G. Alverson, E. Barberis, C. Freer, Y. Haddad, A. Hortiangtham, J. Li, G. Madigan, B. Marzocchi, D.M. Morse, V. Nguyen, T. Orimoto, A. Parker, L. Skinnari, A. TishelmanCharny, T. Wamorkar, B. Wang, A. Wisecarver, D. Wood

Northwestern University, Evanston, U.S.A.

S. Bhattacharya, J. Bueghly, Z. Chen, A. Gilbert, T. Gunter, K.A. Hahn, N. Odell, M.H. Schmitt, K. Sung, M. Velasco

University of Notre Dame, Notre Dame, U.S.A.

R. Bucci, N. Dev, R. Goldouzian, M. Hildreth, K. Hurtado Anampa, C. Jessop, K. Lannon, N. Loukas, N. Marinelli, I. Mcalister, F. Meng, K. Mohrman, Y. Musienko ${ }^{50}$, R. Ruchti, P. Siddireddy, M. Wayne, A. Wightman, M. Wolf, L. Zygala

The Ohio State University, Columbus, U.S.A.

J. Alimena, B. Bylsma, B. Cardwell, L.S. Durkin, B. Francis, C. Hill, A. Lefeld, B.L. Winer, B.R. Yates

Princeton University, Princeton, U.S.A.

F.M. Addesa, B. Bonham, P. Das, G. Dezoort, P. Elmer, A. Frankenthal, B. Greenberg, N. Haubrich, S. Higginbotham, A. Kalogeropoulos, G. Kopp, S. Kwan, D. Lange, M.T. Lucchini, D. Marlow, K. Mei, I. Ojalvo, J. Olsen, C. Palmer, D. Stickland, C. Tully

University of Puerto Rico, Mayaguez, U.S.A.

S. Malik, S. Norberg

Purdue University, West Lafayette, U.S.A.

A.S. Bakshi, V.E. Barnes, R. Chawla, S. Das, L. Gutay, M. Jones, A.W. Jung, S. Karmarkar, M. Liu, G. Negro, N. Neumeister, C.C. Peng, S. Piperov, A. Purohit, J.F. Schulte, M. Stojanovic ${ }^{18}$, J. Thieman, F. Wang, R. Xiao, W. Xie

Purdue University Northwest, Hammond, U.S.A.

J. Dolen, N. Parashar

Rice University, Houston, U.S.A.

A. Baty, S. Dildick, K.M. Ecklund, S. Freed, F.J.M. Geurts, A. Kumar, W. Li, B.P. Padley,

R. Redjimi, J. Roberts ${ }^{\dagger}$, W. Shi, A.G. Stahl Leiton

University of Rochester, Rochester, U.S.A.

A. Bodek, P. de Barbaro, R. Demina, J.L. Dulemba, C. Fallon, T. Ferbel, M. Galanti,

A. Garcia-Bellido, O. Hindrichs, A. Khukhunaishvili, E. Ranken, R. Taus 
Rutgers, The State University of New Jersey, Piscataway, U.S.A.

B. Chiarito, J.P. Chou, A. Gandrakota, Y. Gershtein, E. Halkiadakis, A. Hart, M. Heindl, E. Hughes, S. Kaplan, O. Karacheban ${ }^{26}$, I. Laflotte, A. Lath, R. Montalvo, K. Nash, M. Osherson, S. Salur, S. Schnetzer, S. Somalwar, R. Stone, S.A. Thayil, S. Thomas, H. Wang

University of Tennessee, Knoxville, U.S.A.

H. Acharya, A.G. Delannoy, S. Spanier

Texas A\&M University, College Station, U.S.A.

O. Bouhali $^{95}$, M. Dalchenko, A. Delgado, R. Eusebi, J. Gilmore, T. Huang, T. Kamon ${ }^{96}$, H. Kim, S. Luo, S. Malhotra, R. Mueller, D. Overton, D. Rathjens, A. Safonov

Texas Tech University, Lubbock, U.S.A.

N. Akchurin, J. Damgov, V. Hegde, S. Kunori, K. Lamichhane, S.W. Lee, T. Mengke, S. Muthumuni, T. Peltola, S. Undleeb, I. Volobouev, Z. Wang, A. Whitbeck

Vanderbilt University, Nashville, U.S.A.

E. Appelt, S. Greene, A. Gurrola, W. Johns, C. Maguire, A. Melo, H. Ni, K. Padeken, F. Romeo, P. Sheldon, S. Tuo, J. Velkovska

University of Virginia, Charlottesville, U.S.A.

M.W. Arenton, B. Cox, G. Cummings, J. Hakala, R. Hirosky, M. Joyce, A. Ledovskoy, A. Li, C. Neu, B. Tannenwald, E. Wolfe

Wayne State University, Detroit, U.S.A.

P.E. Karchin, N. Poudyal, P. Thapa

University of Wisconsin - Madison, Madison, WI, U.S.A.

K. Black, T. Bose, J. Buchanan, C. Caillol, S. Dasu, I. De Bruyn, P. Everaerts, C. Galloni, H. He, M. Herndon, A. Hervé, U. Hussain, A. Lanaro, A. Loeliger, R. Loveless, J. Madhusudanan Sreekala, A. Mallampalli, A. Mohammadi, D. Pinna, A. Savin, V. Shang, V. Sharma, W.H. Smith, D. Teague, S. Trembath-reichert, W. Vetens

$\dagger$ : Deceased

1: Also at Vienna University of Technology, Vienna, Austria

2: Also at Institute of Basic and Applied Sciences, Faculty of Engineering, Arab Academy for Science, Technology and Maritime Transport, Alexandria, Egypt, Alexandria, Egypt

3: Also at Université Libre de Bruxelles, Bruxelles, Belgium

4: Also at IRFU, CEA, Université Paris-Saclay, Gif-sur-Yvette, France

5: Also at Universidade Estadual de Campinas, Campinas, Brazil

6: Also at Federal University of Rio Grande do Sul, Porto Alegre, Brazil

7: Also at UFMS, Nova Andradina, Brazil

8: Also at Nanjing Normal University Department of Physics, Nanjing, China

9: Now at The University of Iowa, Iowa City, U.S.A.

10: Also at University of Chinese Academy of Sciences, Beijing, China

11: Also at Institute for Theoretical and Experimental Physics named by A.I. Alikhanov of NRC 'Kurchatov Institute', Moscow, Russia 
12: Also at Joint Institute for Nuclear Research, Dubna, Russia

13: Also at Cairo University, Cairo, Egypt

14: Also at Helwan University, Cairo, Egypt

15: Now at Zewail City of Science and Technology, Zewail, Egypt

16: Also at Suez University, Suez, Egypt

17: Now at British University in Egypt, Cairo, Egypt

18: Also at Purdue University, West Lafayette, U.S.A.

19: Also at Université de Haute Alsace, Mulhouse, France

20: Also at Ilia State University, Tbilisi, Georgia

21: Also at Erzincan Binali Yildirim University, Erzincan, Turkey

22: Also at CERN, European Organization for Nuclear Research, Geneva, Switzerland

23: Also at RWTH Aachen University, III. Physikalisches Institut A, Aachen, Germany

24: Also at University of Hamburg, Hamburg, Germany

25: Also at Department of Physics, Isfahan University of Technology, Isfahan, Iran, Isfahan, Iran

26: Also at Brandenburg University of Technology, Cottbus, Germany

27: Also at Skobeltsyn Institute of Nuclear Physics, Lomonosov Moscow State University, Moscow, Russia

28: Also at Physics Department, Faculty of Science, Assiut University, Assiut, Egypt

29: Also at Eszterhazy Karoly University, Karoly Robert Campus, Gyongyos, Hungary

30: Also at Institute of Physics, University of Debrecen, Debrecen, Hungary, Debrecen, Hungary

31: Also at Institute of Nuclear Research ATOMKI, Debrecen, Hungary

32: Also at MTA-ELTE Lendület CMS Particle and Nuclear Physics Group, Eötvös Loránd University, Budapest, Hungary, Budapest, Hungary

33: Also at Wigner Research Centre for Physics, Budapest, Hungary

34: Also at IIT Bhubaneswar, Bhubaneswar, India, Bhubaneswar, India

35: Also at Institute of Physics, Bhubaneswar, India

36: Also at G.H.G. Khalsa College, Punjab, India

37: Also at Shoolini University, Solan, India

38: Also at University of Hyderabad, Hyderabad, India

39: Also at University of Visva-Bharati, Santiniketan, India

40: Also at Indian Institute of Technology (IIT), Mumbai, India

41: Also at Deutsches Elektronen-Synchrotron, Hamburg, Germany

42: Also at Sharif University of Technology, Tehran, Iran

43: Also at Department of Physics, University of Science and Technology of Mazandaran, Behshahr, Iran

44: Now at INFN Sezione di Bari ${ }^{a}$, Università di Bari ${ }^{b}$, Politecnico di Bari ${ }^{c}$, Bari, Italy

45: Also at Italian National Agency for New Technologies, Energy and Sustainable Economic Development, Bologna, Italy

46: Also at Centro Siciliano di Fisica Nucleare e di Struttura Della Materia, Catania, Italy

47: Also at Università di Napoli 'Federico II', NAPOLI, Italy

48: Also at Riga Technical University, Riga, Latvia, Riga, Latvia

49: Also at Consejo Nacional de Ciencia y Tecnología, Mexico City, Mexico

50: Also at Institute for Nuclear Research, Moscow, Russia

51: Now at National Research Nuclear University 'Moscow Engineering Physics Institute' (MEPhI), Moscow, Russia

52: Also at St. Petersburg State Polytechnical University, St. Petersburg, Russia

53: Also at University of Florida, Gainesville, U.S.A.

54: Also at Imperial College, London, United Kingdom 
55: Also at P.N. Lebedev Physical Institute, Moscow, Russia

56: Also at California Institute of Technology, Pasadena, U.S.A.

57: Also at Budker Institute of Nuclear Physics, Novosibirsk, Russia

58: Also at Faculty of Physics, University of Belgrade, Belgrade, Serbia

59: Also at Trincomalee Campus, Eastern University, Sri Lanka, Nilaveli, Sri Lanka

60: Also at INFN Sezione di Pavia ${ }^{a}$, Università di Pavia ${ }^{b}$, Pavia, Italy, Pavia, Italy

61: Also at National and Kapodistrian University of Athens, Athens, Greece

62: Also at Universität Zürich, Zurich, Switzerland

63: Also at Ecole Polytechnique Fédérale Lausanne, Lausanne, Switzerland

64: Also at Stefan Meyer Institute for Subatomic Physics, Vienna, Austria, Vienna, Austria

65: Also at Laboratoire d'Annecy-le-Vieux de Physique des Particules, IN2P3-CNRS, Annecyle-Vieux, France

66: Also at Şırnak University, Sirnak, Turkey

67: Also at Department of Physics, Tsinghua University, Beijing, China, Beijing, China

68: Also at Near East University, Research Center of Experimental Health Science, Nicosia, Turkey

69: Also at Beykent University, Istanbul, Turkey, Istanbul, Turkey

70: Also at Istanbul Aydin University, Application and Research Center for Advanced Studies (App. \& Res. Cent. for Advanced Studies), Istanbul, Turkey

71: Also at Mersin University, Mersin, Turkey

72: Also at Piri Reis University, Istanbul, Turkey

73: Also at Adiyaman University, Adiyaman, Turkey

74: Also at Ozyegin University, Istanbul, Turkey

75: Also at Izmir Institute of Technology, Izmir, Turkey

76: Also at Necmettin Erbakan University, Konya, Turkey

77: Also at Bozok Universitetesi Rektörlügü, Yozgat, Turkey, Yozgat, Turkey

78: Also at Marmara University, Istanbul, Turkey

79: Also at Milli Savunma University, Istanbul, Turkey

80: Also at Kafkas University, Kars, Turkey

81: Also at Istanbul Bilgi University, Istanbul, Turkey

82: Also at Hacettepe University, Ankara, Turkey

83: Also at Vrije Universiteit Brussel, Brussel, Belgium

84: Also at School of Physics and Astronomy, University of Southampton, Southampton, United Kingdom

85: Also at IPPP Durham University, Durham, United Kingdom

86: Also at Monash University, Faculty of Science, Clayton, Australia

87: Also at Bethel University, St. Paul, Minneapolis, U.S.A., St. Paul, U.S.A.

88: Also at Karamanoğlu Mehmetbey University, Karaman, Turkey

89: Also at Ain Shams University, Cairo, Egypt

90: Also at Bingol University, Bingol, Turkey

91: Also at Georgian Technical University, Tbilisi, Georgia

92: Also at Sinop University, Sinop, Turkey

93: Also at Mimar Sinan University, Istanbul, Istanbul, Turkey

94: Also at Erciyes University, KAYSERI, Turkey

95: Also at Texas A\&M University at Qatar, Doha, Qatar

96: Also at Kyungpook National University, Daegu, Korea, Daegu, Korea 